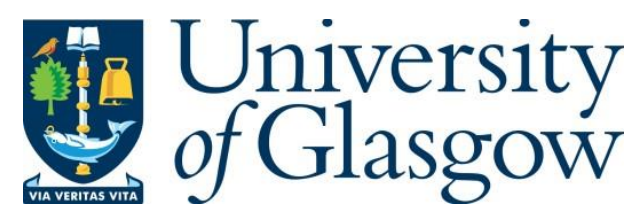

Lee, C. H., Gil, A. J., Greto, G., Kulasegaram, S. and Bonet, J. (2016) A new JamesonSchmidt-Turkel Smooth Particle Hydrodynamics algorithm for large strain explicit fast dynamics. Computer Methods in Applied Mechanics and Engineering, 311, pp. 71-111.

There may be differences between this version and the published version. You are advised to consult the publisher's version if you wish to cite from it.

http://eprints.gla.ac.uk/168848/

Deposited on: 14 September 2018

Enlighten - Research publications by members of the University of Glasgow http://eprints.gla.ac.uk 


\section{Accepted Manuscript}

A new Jameson-Schmidt-Turkel Smooth Particle Hydrodynamics algorithm for large strain explicit fast dynamics

Chun Hean Lee, Antonio J. Gil, Giorgio Greto, Sivakumar

Kulasegaram,

Javier Bonet

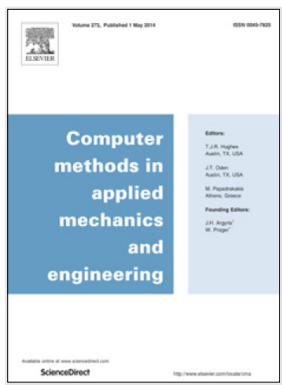

PII:

$$
\text { S0045-7825(16)30418-2 }
$$

DOI:

Reference:

To appear in: Comput. Methods Appl. Mech. Engrg.

Received date: 23 May 2016

Revised date: 19 July 2016

Accepted date: 21 July 2016

Please cite this article as: C.H. Lee, A.J. Gil, G. Greto, S. Kulasegaram, J. Bonet, A new Jameson-Schmidt-Turkel Smooth Particle Hydrodynamics algorithm for large strain explicit fast dynamics, Comput. Methods Appl. Mech. Engrg. (2016), http://dx.doi.org/10.1016/j.cma.2016.07.033

This is a PDF file of an unedited manuscript that has been accepted for publication. As a service to our customers we are providing this early version of the manuscript. The manuscript will undergo copyediting, typesetting, and review of the resulting proof before it is published in its final form. Please note that during the production process errors may be discovered which could affect the content, and all legal disclaimers that apply to the journal pertain. 


\title{
A new Jameson-Schmidt-Turkel Smooth Particle Hydrodynamics algorithm for large strain explicit fast dynamics
}

\author{
Chun Hean Lee ${ }^{a, 1}$, Antonio J. Gil ${ }^{a, 2}$, Giorgio Greto ${ }^{b}$, Sivakumar \\ Kulasegaram $^{b}$, Javier Bonet ${ }^{c}$ \\ (a) Zienkiewicz Centre for Computational Engineering, College of Engineering \\ Swansea University, Bay Campus, SA1 8EN, United Kingdom \\ (b) School of Engineering, Cardiff University, Cardiff CF24 3AA, United Kingdom \\ (c) University of Greenwich, London, SE10 9LS, United Kingdom
}

\begin{abstract}
This paper presents a new Smooth Particle Hydrodynamics (SPH) computational framework for large strain explicit solid dynamics. A mixed-based set of Total Lagrangian conservation laws $[1,2]$ is presented in terms of the linear momentum and an extended set of geometric strain measures, comprised of the deformation gradient, its co-factor and the Jacobian. Taking advantage of this representation, the main aim of this paper is the adaptation of the very efficient Jameson-Schmidt-Turkel (JST) algorithm [3], extensively used in computational fluid dynamics, to a SPH based discretisation of the mixed-based set of conservation laws, with three key distinct novelties. First, a conservative JST-based SPH computational framework is presented with emphasis in nearly incompressible materials. Second, the suppression of numerical instabilities associated with the non-physical zero-energy modes is addressed through a well-established stabilisation procedure. Third, the use of a discrete angular momentum projection algorithm is presented in conjunction with a monolithic Total Variation Diminishing Runge-Kutta time integrator in order to guarantee the global conservation of angular momentum. For completeness, exact enforcement of essential boundary conditions is incorporated through the use of a Lagrange multiplier projection technique.
\end{abstract}

\footnotetext{
${ }^{1}$ Corresponding author: c.h.lee@swansea.ac.uk

${ }^{2}$ Corresponding author: a.j.gil@swansea.ac.uk
} 
A series of challenging numerical examples (e.g. in the near incompressibility regime) are examined in order to assess the robustness and accuracy of the proposed algorithm. The obtained results are benchmarked against a wide spectrum of alternative numerical strategies.

Keywords: Conservation laws, SPH, Instability, JST, Fast dynamics, Incompressibility

\section{Introduction}

Dynamic explicit displacement-based Finite Element codes [4-6] are extensively used for the simulation of large strain dynamic problems in the aerospace, automotive, manufacturing and defence industries. Specifically, the very efficient non-Ladyzhenskaya-Babuska-Brezzi (non-LBB) compliant B-bar hexahedral element $[7,8]$ is one of the preferred options in industry when attempting to model deformable solids experiencing extremely large deformations. However, many practical applications can involve geometries that are far too complex to be meshed using hexahedral elements [9]. In addition, the presence of large deformations accompanied by severe mesh distortion [10] may lead to poorly shaped elements unless some form of adaptive remeshing is applied $[11,12]$.

Over the past decades, an alternative Lagrangian-based Smooth Particle Hydrodynamics (SPH) formalism, originally applied to astrophysical and cosmological problems [13, 14], has been adapted to solid mechanics [15-19]. One of the most attractive features of SPH is its mesh free nature, not requiring the use of an underlying grid. The absence of mesh and the calculation of the interactions among particles based exclusively on their separation allow ease of computation for large deformation problems. For its low computational cost, reasonable accuracy and stability, as well as its ability to handle extremely large distortions [17], the SPH methodology has been shown to be very competitive [10].

As it is well established, the standard displacement-based SPH methodology typically suffers from a number of shortcomings. First, the presence of numerical errors near boundaries due to lack of consistency [20, 21]. Second, the presence of tensile instability which can result in the non-physical clumping of particles [22]. Third, the presence of zero-energy modes due to the rank-deficiency inherent to the use of Galerkin particle integration [23]. 
Fourth, reduced order of convergence for derived variables (i.e. stresses and strains) [24-27].

To rectify these shortcomings, significant efforts have been undertaken to enhance the robustness of the displacement-based SPH method. Specifically, corrections have been applied in order to ensure reproducibility of complete polynomials in finite domains, as well as to pass the patch test. Without being exhaustive, Liu et al. [28] presented an in-depth discussion about reproducibility properties of the SPH method; Johnson et al. [16] proposed a normalised smoothing technique; Chen et al. [29] introduced corrected kernel approximations based upon a Taylor series expansion; Bonet and Kulasegaram [30] introduced corrections in the kernel functions and in their derivatives. However, as reported in [17, 19], enhanced SPH methodologies still suffer from persistent artificial mechanisms similar to hourglassing, especially when dealing with near incompressible behaviour. These numerical instabilities can be alleviated through the use of artificial numerical stabilisation. Some interesting work has been reported in [31-33] where a Taylor-Galerkin stabilisation algorithm is employed for the description of a viscoplastic continuum. However, it is still not yet clear how to introduce appropriate numerical viscosity (leading to a robust framework) through a well-established stabilisation procedure (especially in the context of a nondissipative reversible process). Within the Total Lagrangian formalism, recent work [34] establishes interesting similarities between the SPH framework and that of Peridynamics.

In the Computational Fluid Dynamics (CFD) community, a large variety of stabilised numerical methods [35] have been successfully employed over the years. For instance, Finite Volume based techniques, combined with either an upwinding Riemann solver [36, 37] or a Jameson-Schmidt-Turkel (JST) stabilisation procedure [3, 38]. In order to adapt these approaches to the field of solid dynamics, it is first necessary to reformulate the governing equations in the form of a system of first order conservation laws, as presented in References [1, 2, 31, 37, 39-45, 45-47].

One of the earliest attempts at employing a mixed-based system of conservation laws in solid dynamics can be traced back to the work of Trangenstein and Colella [39, 40], where the primary variables were the linear momentum $\boldsymbol{p}$ and the deformation gradient tensor $\boldsymbol{F}$. Over the last few years, some of the authors of this paper have pursued the same $\{\boldsymbol{p}, \boldsymbol{F}\}$ mixed conservationbased methodology spatially discretised using a wide variety of second order Finite Volume and Finite Element techniques. For instance, an Upwind 
Cell Centred Finite Volume Method (Upwind-CCFVM) [36, 37], a JamesonSchmidt-Turkel Vertex Centred Finite Volume Method (JST-VCFVM) [43], an Upwind Vertex Centred Finite Volume Method (Upwind-VCFVM) [44], a two step Taylor-Galerkin Finite Element Method [45] and a stabilised PetrovGalerkin Finite Element Method (PG-FEM) [41] have been introduced.

The two field $\{\boldsymbol{p}, \boldsymbol{F}\}$ mixed formulation was later augmented by including a new conservation law for the Jacobian of the deformation $J$ to effectively solve nearly and truly incompressible materials [42]. Further enhancement of this $\{\boldsymbol{p}, \boldsymbol{F}, J\}$ framework has also been recently reported in References $[1,2]$ when considering compressible, nearly incompressible and truly incompressible materials governed by a polyconvex constitutive law. In particular, this is important when the co-factor $\boldsymbol{H}$ of the deformation plays a dominant role leading to an extended set $\{\boldsymbol{p}, \boldsymbol{F}, \boldsymbol{H}, J\}$ system. The full $\{\boldsymbol{p}, \boldsymbol{F}, \boldsymbol{H}, J\}$ system can then be reformulated to an alternative description written in terms of entropy conjugates $[1,2]$ due to the existence of a generalised convex entropy function.

In this paper, and following References [1, 2], a stabilised mixed-based $\{\boldsymbol{p}, \boldsymbol{F}, \boldsymbol{H}, J\}$ Total Lagrangian SPH computational framework is presented. One of the main novelties in this paper is the introduction of a well-established stabilisation procedure by taking advantage of the nature of the mixed-based set of equations. In particular, a globally conservative JST stabilisation procedure is introduced. The proposed SPH framework is shown to effectively eliminate the appearance of spurious hourglass-like modes, tensile instability and non-physical pressure instabilities. One of the objectives of this paper is to lay the foundation for a new SPH approach with the final aim to handle in the near future large plastic deformations, shock dominated problems and complex fracture propagation with multi-materials. In addition, to the best of the authors' knowledge, the majority of the available SPH schemes suffer from non-physical pressure instabilities when attempting to model problems with predominant nearly incompressible behaviour $\left(\frac{\kappa}{\mu}>100\right)$ in the context of solid dynamics. The current framework will be shown to perform extremely well in the near incompressible regime for the entire range of deformations and opens up interesting possibilities for modelling in the field of biomechanics, where this consideration is very relevant.

The outline of the paper is broken down as follows. Section 2 revisits the complete mixed-based $\{\boldsymbol{p}, \boldsymbol{F}, \boldsymbol{H}, J\}$ set of first order conservation laws supplemented with an appropriate constitutive law. Section 3 describes the computational methodology of the SPH framework. Variational statement 


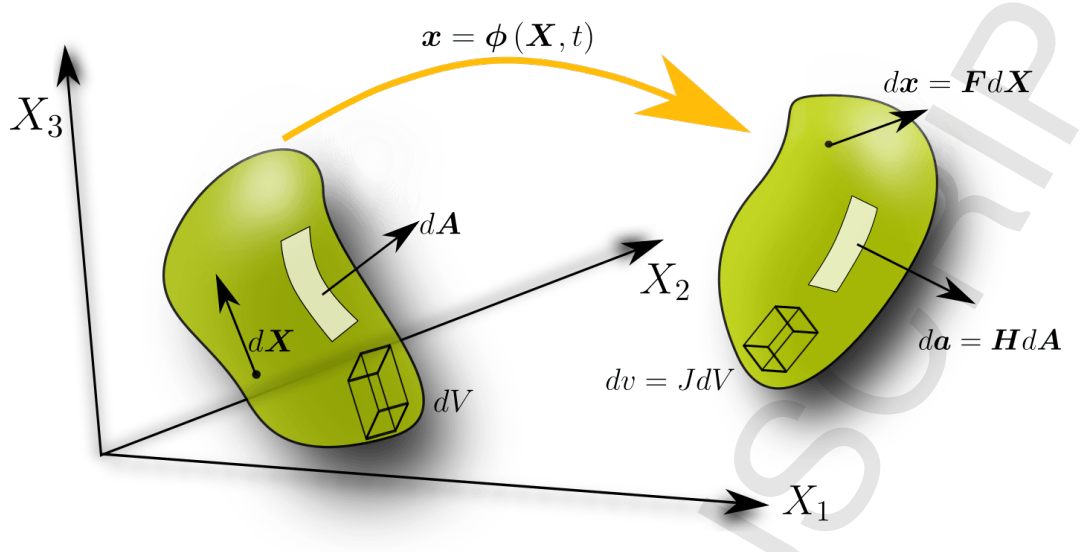

Figure 1: Motion of a continuum domain

of the mixed-based $\{\boldsymbol{p}, \boldsymbol{F}, \boldsymbol{H}, J\}$ system, SPH spatial discretisation and an adapted JST stabilisation term are also presented. For clarity, the complete JST-SPH flowchart is summarised in Section 4. Section 5 describes the TVD Runge-Kutta time integrator used for temporal discretisation. Section 6 describes a monolithic (least-square) projection algorithm used to globally preserve the angular momentum. Section 7 presents the algorithmic description of the proposed $\{\boldsymbol{p}, \boldsymbol{F}, \boldsymbol{H}, J\}$ JST-SPH methodology. In Section 8, an extensive set of challenging numerical examples is presented to assess the performance of the proposed methodology. Finally, Section 9 presents some concluding remarks and current directions of research.

\section{Reversible elastodynamics}

Consider the three dimensional deformation of an elastic body moving from its initial configuration occupying a volume $V$, of boundary $\partial V$, to a current configuration at time $t$ occupying a volume $v$, of boundary $\partial v$ (see Figure 1). The motion is defined through a deformation mapping $\boldsymbol{x}=$ $\boldsymbol{\phi}(\boldsymbol{X}, t)$ which satisfies the following set of (Total Lagrangian) conservation laws [37, 41, 43, 45-47]: 


$$
\begin{aligned}
& \frac{\partial \boldsymbol{p}}{\partial t}-\mathrm{DIV} \boldsymbol{P}=\boldsymbol{f}_{0} ; \\
& \frac{\partial \boldsymbol{F}}{\partial t}-\nabla_{0}\left(\frac{\boldsymbol{p}}{\rho_{0}}\right)=\mathbf{0} \\
& \frac{\partial \boldsymbol{H}}{\partial t}-\operatorname{CURL}\left(\frac{\boldsymbol{p}}{\rho_{0}} \times \boldsymbol{F}\right)=\mathbf{0} ; \\
& \frac{\partial J}{\partial t}-\operatorname{DIV}\left(\boldsymbol{H}^{T} \frac{\boldsymbol{p}}{\rho_{0}}\right)=0 .
\end{aligned}
$$

Here, $\boldsymbol{p}$ represents the linear momentum per unit of undeformed volume, $\rho_{0}$ is the material density, $\boldsymbol{F}$ is the deformation gradient (or fibre map), $\boldsymbol{H}$ is the co-factor of the deformation (or area map), $J$ is the Jacobian of the deformation (or volume map), $\boldsymbol{P}$ is the first Piola-Kirchhoff stress tensor and $\boldsymbol{f}_{0}$ is a material body force term. The symbol $\mathbf{x}$ represents the tensor cross product between vectors and/or second order tensors in the sense of [1, 2, 48, 49], DIV and CURL represent the material divergence and curl operators $^{3}[2]$ and $\boldsymbol{\nabla}_{0}$ represents the material gradient operator defined as $\boldsymbol{\nabla}_{0} \equiv \frac{\partial}{\partial \boldsymbol{X}}$. The above system (1) can alternatively be written in a concise manner as:

$$
\frac{\partial \mathcal{U}}{\partial t}+\frac{\partial \mathcal{F}_{I}}{\partial X_{I}}=\mathcal{S} ; \quad \forall I=1,2,3
$$

where $\mathcal{U}$ denotes a set of conservation variables, $\mathcal{S}$ represents the source term and $\mathcal{F}_{I}$ represents the flux vector in the Cartesian direction $I$, namely:

$$
\mathcal{U}=\left[\begin{array}{c}
\boldsymbol{p} \\
\boldsymbol{F} \\
\boldsymbol{H} \\
J
\end{array}\right] ; \quad \mathcal{F}_{I}=\left[\begin{array}{c}
\boldsymbol{P} \boldsymbol{E}_{I} \\
\frac{\boldsymbol{p}}{\rho_{0}} \otimes \boldsymbol{E}_{I} \\
\boldsymbol{F} \times\left(\frac{\boldsymbol{p}}{\rho_{0}} \otimes \boldsymbol{E}_{I}\right) \\
\boldsymbol{H}:\left(\frac{\boldsymbol{p}}{\rho_{0}} \otimes \boldsymbol{E}_{I}\right)
\end{array}\right] ; \quad \mathcal{S}=\left[\begin{array}{c}
\boldsymbol{f}_{0} \\
\mathbf{0} \\
\mathbf{0} \\
0
\end{array}\right]
$$

with $\boldsymbol{E}_{I}$ is the $I^{\text {th }}$ unit vector of the Cartesian basis defined as:

$$
\boldsymbol{E}_{1}=\left[\begin{array}{l}
1 \\
0 \\
0
\end{array}\right] ; \quad \boldsymbol{E}_{2}=\left[\begin{array}{l}
0 \\
1 \\
0
\end{array}\right] ; \quad \boldsymbol{E}_{3}=\left[\begin{array}{l}
0 \\
0 \\
1
\end{array}\right]
$$

\footnotetext{
${ }^{3}$ The material divergence of a second order two-point tensor $\boldsymbol{A}$ is defined as $[\mathrm{DIV} \boldsymbol{A}]_{i}=$ $\frac{\partial A_{i I}}{\partial X_{I}}$, whereas the material CURL is defined by $[\mathrm{CURL} \boldsymbol{A}]_{i I}=\mathcal{E}_{I J K} \frac{\partial A_{i K}}{\partial X_{J}}$ in terms of the third order alternating tensor $\mathcal{E}_{I J K}$.
} 
Notice that in the presence of non-smooth solutions, the above system (1) of local conservation laws must be accompanied by suitable jump conditions as described in References [1, 36, 37, 43, 44]. Moreover, it is clear from expressions (1b) and (1c) that two sets of involutions [50] need to be satisfied by the conservation variables $\boldsymbol{F}$ and $\boldsymbol{H}$, that is:

$$
\mathrm{CURL} \boldsymbol{F}=\mathbf{0} ; \quad \operatorname{DIVH}=\mathbf{0} .
$$

Using the involutions described above, the area (1c) and volume map (1d) conservation equations can be further reduced to:

$$
\frac{\partial \boldsymbol{H}}{\partial t}-\boldsymbol{F} \times \boldsymbol{\nabla}_{0}\left(\frac{\boldsymbol{p}}{\rho_{0}}\right)=\mathbf{0} ; \quad \frac{\partial J}{\partial t}-\boldsymbol{H}: \boldsymbol{\nabla}_{0}\left(\frac{\boldsymbol{p}}{\rho_{0}}\right)=0 .
$$

The current geometry $\boldsymbol{x}$ can be recovered through time integration of the linear momentum $\boldsymbol{p}$ as

$$
\frac{\partial \boldsymbol{x}}{\partial t}=\frac{\boldsymbol{p}}{\rho_{0}} .
$$

In the especial case of an isothermal reversible deformation process, the closure of system (1) requires the introduction of a thermodynamically consistent [51] and material frame indifferent [37, 42] constitutive law relating the stress tensor $\boldsymbol{P}$ and the strain measures $\{\boldsymbol{F}, \boldsymbol{H}, J\}$. Finally, for the complete definition of the Initial Boundary Value Problem (IBVP), initial and boundary (essential and natural) conditions must also be specified as appropriate.

Remark 1: Inevitably, there is a significant amount of redundancy in the above $\{\boldsymbol{p}, \boldsymbol{F}, \boldsymbol{H}, J\}$ mixed-set of equations (1). References [36, 37, 41-45] have considered simpler mixed-based systems $\{\boldsymbol{p}, \boldsymbol{F}\}$ and $\{\boldsymbol{p}, \boldsymbol{F}, J\}$. These reduced mixed-based systems have proved robust. In general, however, only the full system is capable of being symmetrised $[1,2]$ when expressed in terms of entropy-based variables [2]. Finally, it is important that the computational technique employed to discretise any of the proposed mixed-based systems ensures the satisfaction of the relevant involutions [36, 37, 41-43, 45].

\subsection{Polyconvexity: Nearly incompressible polyconvex model}

For the particular case of a polyconvex nearly incompressible MooneyRivlin material, the strain energy $W$ can be additively decomposed into 
deviatoric $\hat{W}(\boldsymbol{F}, \boldsymbol{H}, J)$ and volumetric $U(J)$ contributions as [49]:

$W=\hat{W}+U ; \quad \hat{W}=\varsigma J^{-2 / 3}(\boldsymbol{F}: \boldsymbol{F})+\xi J^{-2}(\boldsymbol{H}: \boldsymbol{H})^{3 / 2} ; \quad U=\frac{\kappa}{2}(J-1)^{2}$,

where $\varsigma, \xi$ and $\kappa$ (bulk modulus) are positive material parameters. By comparison of the tangent elasticity operator at the initial undeformed configuration with that of classical linearised elasticity [2], appropriate values for the material parameters $\varsigma$ and $\xi$ can be defined in terms of the shear modulus $\mu$, that is, $2 \varsigma+3 \sqrt{3} \xi=\mu$. It is worthwhile noticing how the deviatoric component $\hat{W}$ of the strain energy depends explicitly on the Jacobian $J$. As already stated in [49], this arises as a consequence of the polyconvex requirement imposed on the constitutive model.

The conjugate stresses [2] (see Section 3 on pg. 149) yield:

$$
\boldsymbol{\Sigma}_{\boldsymbol{F}}=\frac{\partial \hat{W}}{\partial \boldsymbol{F}}=2 \varsigma J^{-2 / 3} \boldsymbol{F} ; \quad \boldsymbol{\Sigma}_{\boldsymbol{H}}=\frac{\partial \hat{W}}{\partial \boldsymbol{H}}=3 \xi J^{-2}(\boldsymbol{H}: \boldsymbol{H})^{1 / 2} \boldsymbol{H},
$$

and

$$
\Sigma_{J}=\hat{\Sigma}_{J}+p ; \quad \hat{\Sigma}_{J}=\frac{\partial \hat{W}}{\partial J}=-\frac{2 \varsigma}{3} J^{-5 / 3}(\boldsymbol{F}: \boldsymbol{F})-2 \xi J^{-3}(\boldsymbol{H}: \boldsymbol{H})^{3 / 2},
$$

where $p$ represents the hydrostatic pressure field defined by

$$
p=\frac{d U}{d J}=\kappa(J-1) .
$$

Note that the conjugate stress $\Sigma_{J}$ consists of both a deviatoric contribution $\hat{\Sigma}_{J}$ and a volumetric (or pressure) contribution $p$. For $\xi=0$, the MooneyRivlin model described above (8) degenerates into the so-called nearly incompressible neo-Hookean model.

Following References [2, 48], it is then possible to express the first PiolaKirchhoff stress tensor $\boldsymbol{P}$ in terms of the geometric strains $\{\boldsymbol{F}, \boldsymbol{H}, J\}$ and conjugate stresses $\left\{\boldsymbol{\Sigma}_{\boldsymbol{F}}, \boldsymbol{\Sigma}_{\boldsymbol{H}}, \Sigma_{J}\right\}$ as:

$$
\boldsymbol{P}=\Sigma_{\boldsymbol{F}}+\boldsymbol{\Sigma}_{\boldsymbol{H}} \times \boldsymbol{F}+\Sigma_{J} \boldsymbol{H} .
$$

Remark 2: Satisfaction of polyconvexity is a sufficient, but not necessary, 
condition to guarantee existence of real wave speeds. For instance, for the reduced mixed-based systems $\{\boldsymbol{p}, \boldsymbol{F}\}$ and $\{\boldsymbol{p}, \boldsymbol{F}, J\}$, the strain energy potentials described in Reference [37] (see Equation (9) on pg. 16 for $\{\boldsymbol{p}, \boldsymbol{F}\}$ system) and Reference [42] (see Equation (42) on pg. 669 for $\{\boldsymbol{p}, \boldsymbol{F}, J\}$ system) do not in general fulfil the polyconvexity condition. However, these strain energy potentials have been shown to satisfy rank one convexity, hence guaranteeing the existence of physical waves propagating throughout the domain [42].

\section{Spatial discretisation}

The set of local conservation equations described above (1) has already been (spatially) discretised by the authors [1, 2, 36, 37, 41-44] using a variety of numerical techniques, namely the Petrov-Galerkin Finite Element method $[1,2,41,42]$, the two step Taylor-Galerkin Finite Element method [45], the Vertex Centred Upwind Finite Volume method [44], the Vertex Centred Jameson-Schmidt-Turkel (JST) Finite Volume method [43] and the Cell Centred Upwind Finite Volume method [36, 37]. In this section, we present a new stabilised Smooth Particle Hydrodynamics (SPH) framework tailor-made to the mixed-based set of Total Lagrangian conservation laws $\{\boldsymbol{p}, \boldsymbol{F}, \boldsymbol{H}, J\}(1)$.

\subsection{General remarks}

In general, a standard weak statement of the above mixed-based system $\{\boldsymbol{p}, \boldsymbol{F}, \boldsymbol{H}, J\}(1 \mathrm{a}-1 \mathrm{~d})$ is established by the integral condition [1, 2]:

$$
0=\int_{V} \delta \mathcal{V}^{T} \mathcal{R} d V=\int_{V}\left(\delta \boldsymbol{v} \cdot \boldsymbol{\mathcal { R }}_{\boldsymbol{p}}+\delta \boldsymbol{\Sigma}_{\boldsymbol{F}}: \boldsymbol{\mathcal { R }}_{\boldsymbol{F}}+\delta \boldsymbol{\Sigma}_{\boldsymbol{H}}: \boldsymbol{\mathcal { R }}_{\boldsymbol{H}}+\delta \Sigma_{J} \mathcal{R}_{J}\right) d V
$$

for all conjugate pairs $\delta \mathcal{V}=\left[\delta \boldsymbol{v}, \delta \boldsymbol{\Sigma}_{\boldsymbol{F}}, \delta \boldsymbol{\Sigma}_{\boldsymbol{H}}, \delta \Sigma_{J}\right]^{T}$ compatible with the boundary conditions. Here, $\delta \boldsymbol{v}$ represents the virtual velocity, $\left\{\delta \boldsymbol{\Sigma}_{\boldsymbol{F}}, \delta \boldsymbol{\Sigma}_{\boldsymbol{H}}, \delta \Sigma_{J}\right\}$ 
represent virtual conjugate stresses [1] and the residual $\mathcal{R}$ is expressed as

$$
\mathcal{R}=\left[\begin{array}{c}
\mathcal{R}_{p} \\
\mathcal{R}_{\boldsymbol{F}} \\
\mathcal{R}_{\boldsymbol{H}} \\
\mathcal{R}_{J}
\end{array}\right]=\left[\begin{array}{c}
\frac{\partial \boldsymbol{p}}{\partial t}-\operatorname{DIV} \boldsymbol{P}-\boldsymbol{f}_{0} \\
\frac{\partial \boldsymbol{F}}{\partial t}-\boldsymbol{\nabla}_{0}\left(\frac{\boldsymbol{p}}{\rho_{0}}\right) \\
\frac{\partial \boldsymbol{H}}{\partial t}-\boldsymbol{F} \times \boldsymbol{\nabla}_{0}\left(\frac{\boldsymbol{p}}{\rho_{0}}\right) \\
\frac{\partial J}{\partial t}-\boldsymbol{H}: \boldsymbol{\nabla}_{0}\left(\frac{\boldsymbol{p}}{\rho_{0}}\right)
\end{array}\right]
$$

In order to obtain the corresponding weak form for each conservation law, the term containing the virtual velocity field $\delta \boldsymbol{v}$ is first given by:

$$
\int_{V} \delta \boldsymbol{v} \cdot \boldsymbol{\mathcal { R }}_{\boldsymbol{p}} d V=0
$$

Upon substitution of $\boldsymbol{\mathcal { R }}_{\boldsymbol{p}}$ (14a) into expression (15), and after the application of integration by parts, it gives:

$$
\underbrace{\int_{V} \delta \boldsymbol{v} \cdot \frac{\partial \boldsymbol{p}}{\partial t} d V}_{\delta \mathcal{W}_{\text {iner }}}=\underbrace{\int_{\partial V} \delta \boldsymbol{v} \cdot \boldsymbol{t} d A+\int_{V} \delta \boldsymbol{v} \cdot \boldsymbol{f}_{0} d V}_{\delta \mathcal{W}_{\text {ext }}}-\underbrace{\int_{V} \boldsymbol{P}: \boldsymbol{\nabla}_{0} \delta \boldsymbol{v} d V}_{\delta \mathcal{W}_{\text {int }}}
$$

Here, $\delta \mathcal{W}_{\text {iner }}$ represents the inertial virtual work and $\delta \mathcal{W}_{\text {ext }}$ and $\delta \mathcal{W}_{\text {int }}$ represent the external and internal virtual works, respectively. As it is well known, the main advantage of carrying out integration by parts, as shown above, is to naturally enable the imposition of possible boundary tractions $\boldsymbol{t}[1,2]$.

Analogously, by grouping individual conjugate stress components $\left\{\delta \boldsymbol{\Sigma}_{\boldsymbol{F}}, \delta \boldsymbol{\Sigma}_{\boldsymbol{H}}, \delta \Sigma_{J}\right\}$, the three geometric conservation laws can now be formulated as:

$$
\begin{aligned}
\int_{V} \delta \boldsymbol{\Sigma}_{\boldsymbol{F}}: \frac{\partial \boldsymbol{F}}{\partial t} d V & =\int_{V} \delta \boldsymbol{\Sigma}_{\boldsymbol{F}}:\left[\boldsymbol{\nabla}_{0}\left(\frac{\boldsymbol{p}}{\rho_{0}}\right)\right] d V \\
\int_{V} \delta \boldsymbol{\Sigma}_{\boldsymbol{H}}: \frac{\partial \boldsymbol{H}}{\partial t} d V & =\int_{V} \delta \boldsymbol{\Sigma}_{\boldsymbol{H}}:\left[\boldsymbol{F} \times \boldsymbol{\nabla}_{0}\left(\frac{\boldsymbol{p}}{\rho_{0}}\right)\right] d V ; \\
\int_{V} \delta \Sigma_{J} \frac{\partial J}{\partial t} d V & =\int_{V} \delta \Sigma_{J}\left[\boldsymbol{H}: \boldsymbol{\nabla}_{0}\left(\frac{\boldsymbol{p}}{\rho_{0}}\right)\right] d V .
\end{aligned}
$$




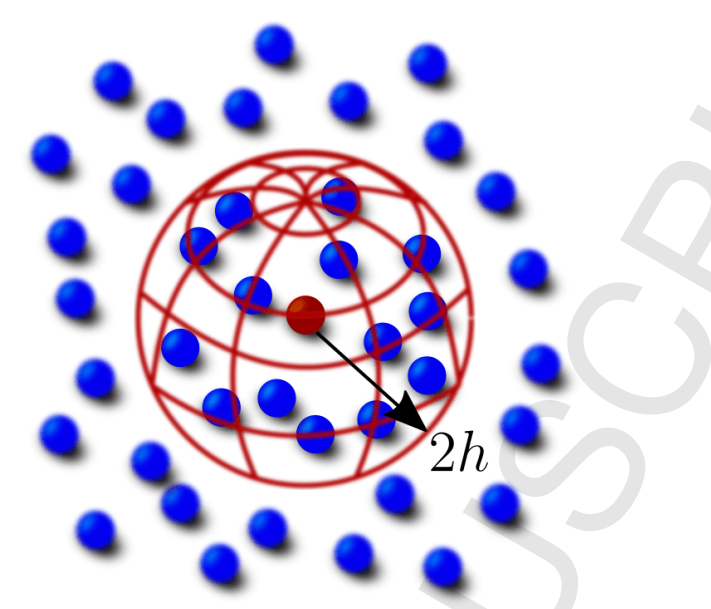

Figure 2: Particle approximation

\subsection{Corrected SPH approximation}

Consider the elastic body is described (discretised) by a cloud of particles as shown in Figure 2. In the context of mesh free methods [17, 18, 30, 52], the problem variables $\{\boldsymbol{p}, \boldsymbol{F}, \boldsymbol{H}, J\}$ are in general interpolated at any given position (quadrature point) via corrected kernel (or smoothing) functions $\tilde{W}$ with a given compact support of radius $2 h$ around every particle. For a given position $\boldsymbol{X}_{a}$, the problem variables can be expanded as

$$
\left[\begin{array}{c}
\boldsymbol{p}\left(\boldsymbol{X}_{a}, t\right) \\
\boldsymbol{F}\left(\boldsymbol{X}_{a}, t\right) \\
\boldsymbol{H}\left(\boldsymbol{X}_{a}, t\right) \\
J\left(\boldsymbol{X}_{a}, t\right)
\end{array}\right] \approx \sum_{b \in \Lambda_{a}^{b}} V_{b} \tilde{W}_{b}\left(\boldsymbol{X}_{a}\right)\left[\begin{array}{c}
\boldsymbol{p}_{b} \\
\boldsymbol{F}_{b} \\
\boldsymbol{H}_{b} \\
J_{b}
\end{array}\right]
$$

Here, $\Lambda_{a}^{b}$ represents the set of neighbouring particles $b$ that lie inside a sphere of a given radius around $\boldsymbol{X}_{a}$ and $V_{b}$ and $\left\{\boldsymbol{p}_{b}, \boldsymbol{F}_{b}, \boldsymbol{H}_{b}, J_{b}\right\}$ represent the volume and time varying problem variables stored at particle $b$, respectively. In addition, $\left\{\boldsymbol{p}\left(\boldsymbol{X}_{a}, t\right), \boldsymbol{F}\left(\boldsymbol{X}_{a}, t\right), \boldsymbol{H}\left(\boldsymbol{X}_{a}, t\right), J\left(\boldsymbol{X}_{a}, t\right)\right\}$ represent the problem variables at (quadrature) position $\boldsymbol{X}_{a}$ and time $t$. The use of corrected kernel approximations $\tilde{W}$ ensure that both constant and linear functions are perfectly interpolated (see Section 4.2 in Reference [52] on pg. 105-106).

In this paper, where SPH is employed (i.e. particle integration) and for computational efficiency, further assumptions are made on the interpolation 
described above (18), namely [17, 18, 30]:

$$
\boldsymbol{p}\left(\boldsymbol{X}_{a}, t\right) \approx \boldsymbol{p}_{a} ; \quad \boldsymbol{F}\left(\boldsymbol{X}_{a}, t\right) \approx \boldsymbol{F}_{a} ; \quad \boldsymbol{H}\left(\boldsymbol{X}_{a}, t\right) \approx \boldsymbol{H}_{a} ; \quad J\left(\boldsymbol{X}_{a}, t\right) \approx J_{a}
$$

The above interpolations (19) will be used throughout in Section 3.3 when presenting the spatial discretisation of the above set of equations ((16) and (17)). The exact same assumption is applied for the conjugate pairs $\left\{\delta \boldsymbol{v}, \delta \boldsymbol{\Sigma}_{\boldsymbol{F}}, \delta \boldsymbol{\Sigma}_{\boldsymbol{H}}, \delta \Sigma_{J}\right\}$, that is:

$\delta \boldsymbol{v}\left(\boldsymbol{X}_{a}\right) \approx \delta \boldsymbol{v}_{a} ; \quad \delta \boldsymbol{\Sigma}_{\boldsymbol{F}}\left(\boldsymbol{X}_{a}\right) \approx \delta \boldsymbol{\Sigma}_{\boldsymbol{F}}^{a} ; \quad \delta \boldsymbol{\Sigma}_{\boldsymbol{H}}\left(\boldsymbol{X}_{a}\right) \approx \delta \boldsymbol{\Sigma}_{\boldsymbol{H}}^{a} ; \quad \delta \Sigma_{J}\left(\boldsymbol{X}_{a}\right) \approx \delta \Sigma_{J}^{a}$.

\subsubsection{Gradient evaluation}

For evaluation of the material gradient of any arbitrary vector function $\boldsymbol{f}$, we employ the following approximation which is described $\operatorname{as}^{4}[52]$

$$
\boldsymbol{\nabla}_{0} \boldsymbol{f}\left(\boldsymbol{X}_{a}\right) \approx \sum_{b \in \Lambda_{a}^{b}} V_{b}\left(\boldsymbol{f}_{b}-\boldsymbol{f}_{a}\right) \otimes \tilde{\nabla}_{0} \mathrm{~W}_{b}\left(\boldsymbol{X}_{a}\right)
$$

Specifically, the term $-\boldsymbol{f}_{a}$ is added into the above expression in order to ensure that the gradient vanishes for a uniform field [53]. In addition, the use of the kernel gradient correction $\tilde{\nabla}_{0}$ ensures the gradient of any linear field distribution is exactly evaluated. This type of kernel gradient correction has been extensively discussed in Reference [52] (see Section 4.1 on pg. 105).

Remark 3: It is also possible to describe other gradient evaluations oftenly used in classical displacement-based SPH $[18,53]$. One of the most commonly used expressions for the gradient evaluation is defined by approximating [53]

$$
\boldsymbol{\nabla}_{0} \boldsymbol{f}\left(\boldsymbol{X}_{a}\right) \approx \sum_{b \in \Lambda_{a}^{b}} V_{b}\left(\boldsymbol{f}_{b}-\boldsymbol{f}_{a}\right) \otimes \nabla_{0} \mathrm{~W}_{b}\left(\boldsymbol{X}_{a}\right)
$$

through the use of the uncorrected kernel gradient $\nabla_{0}\left(\right.$ where $\left.\nabla_{0} \neq \tilde{\nabla}_{0}\right)$. It is worth noticing that the above gradient approximation (22) does not render first-order completeness [52].

\footnotetext{
${ }^{4}$ It is interesting to observe that this gradient approximation resembles the well-known least-square gradient reconstruction typically used in the Finite Volume method (see [37], Equation (52) on pg. 26).
} 
Another interesting approach for the gradient approximation can be obtained through the use of the "Corrected Gradient of a Corrected Kernel $\tilde{\nabla}_{0} \tilde{W}^{\prime \prime}[52]$ described as

$$
\boldsymbol{\nabla}_{0} \boldsymbol{f}\left(\boldsymbol{X}_{a}\right) \approx \sum_{b \in \Lambda_{a}^{b}} V_{b} \boldsymbol{f}_{b} \otimes \tilde{\boldsymbol{\nabla}}_{0} \tilde{\mathrm{W}}_{b}\left(\boldsymbol{X}_{a}\right)
$$

As reported in Reference [52] (see Section 4.3 on pg. 106-107), this approximation reproduces exactly the gradient of any constant and/or linear function.

\subsubsection{Laplacian evaluation}

The Jameson-Schmidt-Turkel (JST) stabilisation procedure to be employed in this paper requires the evaluation of the Laplacian (and the biLaplacian) of a solution function [43]. For this purpose, consider the Laplacian of any arbitrary vector function $\boldsymbol{f}$ to be numerically approximated as:

$$
\mathcal{L}\left[\boldsymbol{f}\left(\boldsymbol{X}_{a}\right)\right] \approx \sum_{b \in \Lambda_{a}^{b}} V_{b}\left(\boldsymbol{f}_{b}-\boldsymbol{f}_{a}\right) \tilde{\Delta}_{0} \mathrm{~W}_{b}\left(\boldsymbol{X}_{a}\right)
$$

where $\tilde{\Delta}_{0}$ represents a corrected Laplacian approximation summarised in Appendix A. In contrast to the work of Bonet and Kulasegaram [30] (see Section 4.3 on pg. 1202-1203), the term $-\boldsymbol{f}_{a}$ is included in order to ensure zeroth-order completeness. More interestingly, the above expression (24) has a similar mathematical structure to the non-divided Laplacian evaluation typical of the JST stabilisation for a Finite Volume method, as presented in Reference [43] (see Equation (26) on pg. 678).

It is now possible to approximate the biharmonic operator $\mathcal{L}^{2}$ with the aid of the above Laplacian approximation (24):

$$
\mathcal{L}^{2}\left[\boldsymbol{f}\left(\boldsymbol{X}_{a}\right)\right]:=\mathcal{L}\left[\mathcal{L}\left[\boldsymbol{f}\left(\boldsymbol{X}_{a}\right)\right]\right] \approx \sum_{b \in \Lambda_{a}^{b}} V_{b}\left(\mathcal{L}\left[\boldsymbol{f}\left(\boldsymbol{X}_{b}\right)\right]-\mathcal{L}\left[\boldsymbol{f}\left(\boldsymbol{X}_{a}\right)\right]\right) \tilde{\Delta}_{0} \mathrm{~W}_{b}\left(\boldsymbol{X}_{a}\right)
$$

\subsection{Lagrangian particle technique: Smooth Particle Hydrodynamics}

Typically, in the context of Finite Element [1, 2, 41, 42, 45] and Element Free Galerkin approaches [54-56], the above weak statements (16) and (17) are accurately evaluated using the necessary distribution of quadrature points 
in order to avoid the appearance of non-physical hourglass (or zero-energy) modes $[23,24,57]$. Specifically, the positions of the quadrature points do not in general coincide with those of the material particles $[54,58]$. This is however not the case when employing a SPH discretisation. In the latter case, and for computational efficiency, above integrands (16) and (17) are under-integrated by using the cloud of particles as quadrature points $[15,17$, $19,59,60]$.

This section is devoted to describe the SPH spatial discretisation for the system $\{\boldsymbol{p}, \boldsymbol{F}, \boldsymbol{H}, J\}$ (16) and (17). In particular, Galerkin particle integration (also known as nodal integration [23]), together with the interpolating assumptions (19) and (20), is used.

\subsubsection{Conservation law for linear momentum}

The inertial virtual work $\delta \mathcal{W}_{\text {iner }}(16)$ can be approximated as:

$$
\delta \mathcal{W}_{\text {iner }} \approx \sum_{a} V_{a} \delta \boldsymbol{v}\left(\boldsymbol{X}_{a}\right) \cdot \frac{d \boldsymbol{p}\left(\boldsymbol{X}_{a}, t\right)}{d t} \approx \sum_{a} V_{a} \delta \boldsymbol{v}_{a} \cdot\left[\frac{d \boldsymbol{p}_{a}}{d t}\right]
$$

This clearly leads to the introduction of a lumped mass matrix (without altering the order of convergence of the algorithm [41, 42]) which is usually preferred in the application of explicit fast solid dynamics. The external virtual work $\delta \mathcal{W}_{\text {ext }}$ (comprised of external boundary tractions and body forces) renders:

$$
\begin{aligned}
\delta \mathcal{W}_{\mathrm{ext}} & \approx \sum_{B} A_{B} \delta \boldsymbol{v}\left(\boldsymbol{X}_{B}\right) \cdot \boldsymbol{t}\left(\boldsymbol{X}_{B}, t\right)+\sum_{a} V_{a} \delta \boldsymbol{v}\left(\boldsymbol{X}_{a}\right) \cdot \boldsymbol{f}_{0}^{a} \\
& \approx \sum_{B} A_{B} \delta \boldsymbol{v}_{B} \cdot \boldsymbol{t}_{B}+\sum_{a} V_{a} \delta \boldsymbol{v}_{a} \cdot \boldsymbol{f}_{0}^{a} \\
& \approx \sum_{a} V_{a} \delta \boldsymbol{v}_{a} \cdot \underbrace{\left[\frac{A_{a}}{V_{a}} \boldsymbol{t}_{a}+\boldsymbol{f}_{0}^{a}\right]}_{\boldsymbol{E}_{a}} .
\end{aligned}
$$

In the above expression, $B$ represents a particle placed on the boundary, $A_{B}$ its material tributary area and $\boldsymbol{t}_{B}$ its traction vector computed directly from the given traction boundary conditions or via an appropriate Riemann solver $[35-37,44]$. Notice that in $(27 \mathrm{c}), A_{a}=0$ for those particles not placed on the boundary. 
Finally, the use of Galerkin particle integration on the internal virtual work $\delta \mathcal{W}_{\text {int }}(16)$ contribution gives:

$$
\begin{aligned}
\delta \mathcal{W}_{\mathrm{int}} & \approx \sum_{b} V_{b} \boldsymbol{P}(\underbrace{\boldsymbol{F}\left(\boldsymbol{X}_{b}, t\right)}_{\boldsymbol{F}_{b}}, \underbrace{\boldsymbol{H}\left(\boldsymbol{X}_{b}, t\right)}_{\boldsymbol{H}_{b}}, \underbrace{J\left(\boldsymbol{X}_{b}, t\right)}_{J_{b}}): \nabla_{0} \delta \boldsymbol{v}\left(\boldsymbol{X}_{b}\right) \\
& =\sum_{b} V_{b} \boldsymbol{P}_{b}: \boldsymbol{\nabla}_{0} \delta \boldsymbol{v}\left(\boldsymbol{X}_{b}\right)
\end{aligned}
$$

where $\boldsymbol{P}_{b}:=\boldsymbol{P}\left(\boldsymbol{F}_{b}, \boldsymbol{H}_{b}, J_{b}\right)$ and the interpolating assumptions for $\{\boldsymbol{F}, \boldsymbol{H}, J\}$ described by (19b,c,d) have been used.

With the aid of the gradient evaluation described above (21), equation (28b) can be further expressed in terms of an equivalent force vector $\boldsymbol{T}_{a}$ [19] as:

$$
\begin{aligned}
\delta \mathcal{W}_{\mathrm{int}} & \approx \sum_{b} V_{b} \boldsymbol{P}_{b}: \boldsymbol{\nabla}_{0} \delta \boldsymbol{v}\left(\boldsymbol{X}_{b}\right) \\
& \approx \sum_{b} V_{b} \boldsymbol{P}_{b}:\left(\sum_{a \in \Lambda_{b}^{a}} V_{a}\left(\delta \boldsymbol{v}_{a}-\delta \boldsymbol{v}_{b}\right) \otimes \tilde{\boldsymbol{\nabla}}_{0} \mathrm{~W}_{a}\left(\boldsymbol{X}_{b}\right)\right) \\
& =\sum_{a} V_{a} \delta \boldsymbol{v}_{a} \cdot \underbrace{\left(\sum_{b \in \Lambda_{a}^{b}} V_{b}\left(\boldsymbol{P}_{b} \tilde{\boldsymbol{\nabla}}_{0} \mathrm{~W}_{a}\left(\boldsymbol{X}_{b}\right)-\boldsymbol{P}_{a} \tilde{\boldsymbol{\nabla}}_{0} \mathrm{~W}_{b}\left(\boldsymbol{X}_{a}\right)\right)\right)}_{\boldsymbol{T}_{a}} .
\end{aligned}
$$

Notice that, as discussed in References [19, 34], the above expression (29c) satisfies the global conservation of linear momentum, ensuring that $\sum_{a} V_{a} \boldsymbol{T}_{a}=\mathbf{0}$.

Remark 4: A variety of (globally conservative) equivalent internal force representations $\boldsymbol{T}_{a}$ can be obtained using different numerical approximations for the gradient evaluation $\boldsymbol{\nabla}_{0} \delta \boldsymbol{v}\left(\boldsymbol{X}_{b}\right)$ (28b). Substitution of (22) into (28b) leads to the well-known representation for $\boldsymbol{T}_{a}[22,53,61,62]$, that is

$$
\boldsymbol{T}_{a}=-\sum_{b \in \Lambda_{a}^{b}} V_{b}\left(\boldsymbol{P}_{b}+\boldsymbol{P}_{a}\right) \nabla_{0} \mathrm{~W}_{b}\left(\boldsymbol{X}_{a}\right)
$$

Another concise representation for $\boldsymbol{T}_{a}$ was introduced by Bonet and coworkers $[18,30,52]$ through the use of $\tilde{\nabla}_{0} \tilde{W}(23)$. Substitution of (23) into 
(28b) yields

$$
\boldsymbol{T}_{a}=\sum_{b \in \Lambda_{a}^{b}} V_{b} \boldsymbol{P}_{b} \tilde{\boldsymbol{\nabla}}_{0} \tilde{\mathrm{W}}_{a}\left(\boldsymbol{X}_{b}\right) .
$$

It is also possible to describe other non-globally conservative representations for $\boldsymbol{T}_{a}$ (i.e. $\sum_{a} V_{a} \boldsymbol{T}_{a} \neq \mathbf{0}$ ). The first approach [15] corresponds to expression (30) by replacing $\boldsymbol{\nabla}_{0} \mathrm{~W}_{b}\left(\boldsymbol{X}_{a}\right)$ with $\tilde{\boldsymbol{\nabla}}_{0} \mathrm{~W}_{b}\left(\boldsymbol{X}_{a}\right)$, whereas the second approach [17] corresponds to expression (31) by replacing $\tilde{\boldsymbol{\nabla}}_{0} \tilde{\mathrm{W}}_{a}\left(\boldsymbol{X}_{b}\right)$ with $\tilde{\nabla}_{0} \mathrm{~W}_{a}\left(\boldsymbol{X}_{b}\right)$.

Combination of expressions (26), (27c) and (29c) for any arbitrary virtual velocity field, leads to the following expression:

$$
\frac{d \boldsymbol{p}_{a}}{d t}=\boldsymbol{E}_{a}-\boldsymbol{T}_{a}
$$

\subsubsection{Geometric Conservation laws}

Traditional displacement-based solid mechanics formulations $[15,17-19$, 23, 59] evaluate the strain measures $\{\boldsymbol{F}, \boldsymbol{H}, J\}$ directly from the current geometry alone via $\boldsymbol{F}_{\boldsymbol{x}}:=\boldsymbol{\nabla}_{0} \boldsymbol{x}, \boldsymbol{H}_{\boldsymbol{x}}:=\frac{1}{2} \boldsymbol{\nabla}_{0} \boldsymbol{x} \times \boldsymbol{\nabla}_{0} \boldsymbol{x}$ and $J_{\boldsymbol{x}}=\operatorname{det}\left(\boldsymbol{\nabla}_{0} \boldsymbol{x}\right)$ $[9,24,63]$. However, this approach leads to a formulation in which strains and stresses converge at a rate one order lower than the geometry or displacement. The formulation proposed here is based on deriving independent first order conservation laws for the strain variables [37, 45-47].

Analogously to the discretisation procedure stated in the above Section 3.3.1, the set of geometric conservation laws can now be discretised as:

$$
\begin{aligned}
\frac{d \boldsymbol{F}_{a}}{d t} & =\nabla_{0}\left(\frac{\boldsymbol{p}\left(\boldsymbol{X}_{a}, t\right)}{\rho_{0}}\right) \approx \sum_{b \in \Lambda_{a}^{b}} \frac{V_{b}}{\rho_{0}}\left(\boldsymbol{p}_{b}-\boldsymbol{p}_{a}\right) \otimes \tilde{\boldsymbol{\nabla}}_{0} \mathrm{~W}_{b}\left(\boldsymbol{X}_{a}\right) \\
\frac{d \boldsymbol{H}_{a}}{d t} & =\boldsymbol{F}\left(\boldsymbol{X}_{a}, t\right) \times \nabla_{0}\left(\frac{\boldsymbol{p}\left(\boldsymbol{X}_{a}, t\right)}{\rho_{0}}\right) \approx \boldsymbol{F}_{a} \times \sum_{b \in \Lambda_{a}^{b}} \frac{V_{b}}{\rho_{0}}\left(\boldsymbol{p}_{b}-\boldsymbol{p}_{a}\right) \otimes \tilde{\boldsymbol{\nabla}}_{0} \mathrm{~W}_{b}\left(\boldsymbol{X}_{a}\right) \\
\frac{d J_{a}}{d t} & =\boldsymbol{H}\left(\boldsymbol{X}_{a}, t\right): \boldsymbol{\nabla}_{0}\left(\frac{\boldsymbol{p}\left(\boldsymbol{X}_{a}, t\right)}{\rho_{0}}\right) \approx \boldsymbol{H}_{a}: \sum_{b \in \Lambda_{a}^{b}} \frac{V_{b}}{\rho_{0}}\left(\boldsymbol{p}_{b}-\boldsymbol{p}_{a}\right) \otimes \tilde{\nabla}_{0} \mathrm{~W}_{b}\left(\boldsymbol{X}_{a}\right) .
\end{aligned}
$$

The above mixed-based system ((32) and (33)) will exhibit non-physical numerical instabilities [23, 27, 30,64] when solving large strain solid dynamics problems [41]. To remedy this, an adapted (globally conservative) 
Jameson-Schmidt-Turkel (JST) stabilisation algorithm [43] can be naturally introduced. This will be addressed in the next section.

Remark 5: Notice that, by replacing the velocity field $\frac{p}{\rho_{0}}$ with the current geometry $\boldsymbol{x}$ in (33a), the classical SPH update for the deformation gradient $\boldsymbol{F}_{\boldsymbol{x}}:=\boldsymbol{\nabla}_{0} \boldsymbol{x}$ can be simply recovered:

$$
\boldsymbol{F}_{\boldsymbol{x}}^{a}:=\boldsymbol{\nabla}_{0} \boldsymbol{x}\left(\boldsymbol{X}_{a}\right) \approx \sum_{b \in \Lambda_{a}^{b}} V_{b}\left(\boldsymbol{x}_{b}-\boldsymbol{x}_{a}\right) \otimes \tilde{\boldsymbol{\nabla}}_{0} \mathrm{~W}_{b}\left(\boldsymbol{X}_{a}\right) .
$$

\subsection{Globally conservative Jameson-Schmidt-Turkel (JST) stabilisation}

Within the community of mesh free methods, two well-established families are available for the consideration of numerical stabilisation, namely gradient based stabilisation [23] and the strain smoothing procedure [65, 66]. Unfortunately, to the best of the authors' knowledge, most of the available stabilisation algorithms still suffer from non-physical pressure instabilities in highly nonlinear nearly incompressible scenarios [17, 67].

In this paper, by taking advantage of the conservation structure of the mixed-based set of equations to be solved, numerical stabilisation is introduced via a globally conservative JST stabilisation algorithm (through the use of a blend of Laplacian and biharmonic dissipative operators) [43]. The discrete stabilisation term at particle $a$ for a given conservation variable $\mathcal{U}$ can be written as follows:

$$
\mathcal{D}\left(\mathcal{U}_{a}\right)=\mathcal{D}_{2}\left(\mathcal{U}_{a}\right)+\mathcal{D}_{4}\left(\mathcal{U}_{a}\right)
$$

where the second-order harmonic (or Laplacian) dissipative operator is defined as

$$
\mathcal{D}_{2}\left(\mathcal{U}_{a}\right):=\varepsilon_{\mathcal{U}}^{(2)} c_{p} h_{\min } \mathcal{L}\left[\mathcal{U}\left(\boldsymbol{X}_{a}\right)\right]
$$

and the fourth-order biharmonic dissipative operator (25) as

$$
\begin{aligned}
\mathcal{D}_{4}\left(\mathcal{U}_{a}\right) & :=-\varepsilon_{\mathcal{U}}^{(4)} c_{p} h_{\min }^{3} \mathcal{L}^{2}\left[\mathcal{U}\left(\boldsymbol{X}_{a}\right)\right] \\
& \approx-\varepsilon_{\mathcal{U}}^{(4)} c_{p} h_{\min }^{3} \sum_{b \in \Lambda_{a}^{b}} V_{b}\left(\mathcal{L}\left[\mathcal{U}\left(\boldsymbol{X}_{b}\right)\right]-\mathcal{L}\left[\mathcal{U}\left(\boldsymbol{X}_{a}\right)\right]\right) \tilde{\Delta}_{0} \mathrm{~W}_{b}\left(\boldsymbol{X}_{a}\right)
\end{aligned}
$$

Here, $\varepsilon_{\mathcal{u}}^{(2)}$ and $\varepsilon_{\mathcal{u}}^{(4)}$ are JST parameters which activate either second or fourth order differences for each conservation law, $c_{p}$ represents the $p$-wave speed, 
$h_{\text {min }}$ is the characteristic particle spacing and $\tilde{\Delta}_{0}$ denotes the corrected Laplacian operator [30] (see Section 3.2.2). Following Section 3.2.2, the Laplacian evaluation $\mathcal{L}\left[\mathcal{U}\left(\boldsymbol{X}_{g}\right)\right]$ can be approximated as:

$$
\mathcal{L}\left[\mathcal{U}\left(\boldsymbol{X}_{g}\right)\right] \approx \sum_{h \in \Lambda_{g}^{h}} V_{h}\left(\boldsymbol{U}_{h}-\mathcal{U}_{g}\right) \tilde{\Delta}_{0} \mathrm{~W}_{h}\left(\boldsymbol{X}_{g}\right)
$$

As stated in Reference [43], it is worthwhile pointing out that the nodal JST dissipative term (35) prevents the exact local conservation due to the lack of symmetry of the Laplacian correction, that is $\tilde{\Delta}_{0} \mathrm{~W}_{b}\left(\boldsymbol{X}_{a}\right) \neq \tilde{\Delta}_{0} \mathrm{~W}_{a}\left(\boldsymbol{X}_{b}\right)$. This issue will be addressed later in the paper by using a modified stabilisation term which ensures satisfaction of the conservation of the primary variables (see Section 6).

Remark 6: In general, one of the simplest approaches in order to avoid the appearance of spurious high-frequency modes is to introduce artificial physical viscosity $[41,45]$ through the use of a numerically-based (non-objective) viscous stress $\boldsymbol{P}_{\text {vis }}=\frac{\mu_{\text {vis }}}{\rho_{0}} \nabla_{0} \boldsymbol{p}$ (where $\mu_{\text {vis }}$ represents the numerical shear viscosity with intrinsic time scale and $\rho_{0}$ is the material density). A weak statement for the viscous stabilisation term can be formulated as:

$$
\int_{V} \delta \boldsymbol{v} \cdot \operatorname{DIV} \boldsymbol{P}_{\text {vis }} d V=\int_{V} \delta \boldsymbol{v} \cdot\left(\frac{\mu_{\mathrm{vis}}}{\rho_{0}} \Delta_{0} \boldsymbol{p}\right) d V .
$$

The use of Galerkin particle integration on (39) gives:

$$
\begin{aligned}
\int_{V} \delta \boldsymbol{v} \cdot\left(\frac{\mu_{\mathrm{vis}}}{\rho_{0}} \Delta_{0} \boldsymbol{p}\right) d V & \approx \sum_{a} V_{a} \delta \boldsymbol{v}\left(\boldsymbol{X}_{a}\right) \cdot\left(\frac{\mu_{\mathrm{vis}}}{\rho_{0}} \Delta_{0} \boldsymbol{p}\left(\boldsymbol{X}_{a}, t\right)\right) \\
& \approx \sum_{a} V_{a} \delta \boldsymbol{v}_{a} \cdot\left(\frac{\mu_{\mathrm{vis}}}{\rho_{0}} \Delta_{0} \boldsymbol{p}\left(\boldsymbol{X}_{a}, t\right)\right) \\
& =\sum_{a} V_{a} \delta \boldsymbol{v}_{a} \cdot\left(\varepsilon_{\boldsymbol{p}}^{(2)} c_{p} h_{\min } \sum_{b \in \Lambda_{a}^{b}} V_{b}\left(\boldsymbol{p}_{b}-\boldsymbol{p}_{a}\right) \tilde{\Delta}_{0} \mathrm{~W}_{b}\left(\boldsymbol{X}_{a}\right)\right)
\end{aligned}
$$

with $\varepsilon_{\boldsymbol{p}}^{(2)} c_{p} h_{\text {min }}=\frac{\mu_{\text {vis }}}{\rho_{0}}$. It is clear that the terms inside the parenthesis of (40c) are equivalent to expression (36), but particularised to the case of the linear momentum variable, namely $\mathcal{D}_{2}\left(\boldsymbol{p}_{a}\right)$. 


\section{Complete JST-SPH algorithm}

In this section, we present a summary of the semi-discrete set of governing equations for $\mathcal{U}=[\boldsymbol{p}, \boldsymbol{F}, \boldsymbol{H}, J]^{T}(2)$ :

$$
\begin{aligned}
\frac{d \boldsymbol{p}_{a}}{d t} & =\boldsymbol{E}_{a}-\boldsymbol{T}_{a}+\mathcal{D}\left(\boldsymbol{p}_{a}\right) ; \\
\frac{d \boldsymbol{F}_{a}}{d t} & =\sum_{b \in \Lambda_{a}^{b}} \frac{V_{b}}{\rho_{0}}\left(\boldsymbol{p}_{b}-\boldsymbol{p}_{a}\right) \otimes \tilde{\boldsymbol{\nabla}}_{0} \mathrm{~W}_{b}\left(\boldsymbol{X}_{a}\right)+\mathcal{D}\left(\boldsymbol{F}_{a}\right) ; \\
\frac{d \boldsymbol{H}_{a}}{d t} & =\boldsymbol{F}_{a} \times \sum_{b \in \Lambda_{a}^{b}} \frac{V_{b}}{\rho_{0}}\left(\boldsymbol{p}_{b}-\boldsymbol{p}_{a}\right) \otimes \tilde{\boldsymbol{\nabla}}_{0} \mathrm{~W}_{b}\left(\boldsymbol{X}_{a}\right)+\mathcal{D}\left(\boldsymbol{H}_{a}\right) ; \\
\frac{d J_{a}}{d t} & =\boldsymbol{H}_{a}: \sum_{b \in \Lambda_{a}^{b}} \frac{V_{b}}{\rho_{0}}\left(\boldsymbol{p}_{b}-\boldsymbol{p}_{a}\right) \otimes \tilde{\boldsymbol{\nabla}}_{0} \mathrm{~W}_{b}\left(\boldsymbol{X}_{a}\right)+\mathcal{D}\left(J_{a}\right) .
\end{aligned}
$$

Here, $\left\{\mathcal{D}\left(\boldsymbol{p}_{a}\right), \mathcal{D}\left(\boldsymbol{F}_{a}\right), \mathcal{D}\left(\boldsymbol{H}_{a}\right), \mathcal{D}\left(J_{a}\right)\right\}$ represent the possible JST stabilisation terms. The nodal internal $\boldsymbol{T}_{a}$ and external $\boldsymbol{E}_{a}$ forces are already described in (29c) and (27c), but repeated here for clarity:

$$
\boldsymbol{T}_{a}=\sum_{b \in \Lambda_{a}^{b}} V_{b}\left(\boldsymbol{P}_{b} \tilde{\nabla}_{0} \mathrm{~W}_{a}\left(\boldsymbol{X}_{b}\right)-\boldsymbol{P}_{a} \tilde{\nabla}_{0} \mathrm{~W}_{b}\left(\boldsymbol{X}_{a}\right)\right) ; \quad \boldsymbol{E}_{a}=\frac{A_{a}}{V_{a}} \boldsymbol{t}_{a}+\boldsymbol{f}_{0}^{a}
$$

Similar to $[2,43,44]$, we set $\mathcal{D}\left(\boldsymbol{F}_{a}\right)=\mathcal{D}(\boldsymbol{H})_{a}=\mathbf{0}$ to ensure the discrete satisfaction of the involutions described by equations (5). As a result, only JST stabilisation is applied to the conservation law of the linear momentum $\mathcal{D}\left(\boldsymbol{p}_{a}\right)$ (41a) (i.e. $\varepsilon_{\boldsymbol{p}}^{(2)}$ and $\varepsilon_{\boldsymbol{p}}^{(4)}$ ) and the conservation law of the Jacobian $\mathcal{D}\left(J_{a}\right)(41 \mathrm{~d})$ (i.e. $\varepsilon_{J}^{(2)}$ and $\left.\varepsilon_{J}^{(4)}\right)$. The former alleviates the appearance of spurious zero-energy (hourglass-like [19]) modes and, the latter, removes pressure instabilities when attempting to model problems with predominant nearly incompressible behaviour [42].

As kernel functions do not verify the Kronecker delta property [52, 55], exact enforcement of essential boundary conditions is not as trivial as in the Finite Element method [55]. In this paper, we resort to a simple least-square projection technique for their imposition, summarised in Appendix B for completeness.

\section{Time integration}

Insofar as the resulting set of semi-discrete particle equations (41) is rather large, it will only be suitable to employ an explicit type of time inte- 
grator. For simplicity, an explicit one-step two-stage Total Variation Diminishing Runge-Kutta (TVD-RK) scheme has been used such as that presented in $[1,37,41-44]$. This is described by the following time update equations from time step $t^{n}$ to $t^{n+1}$ :

$$
\begin{aligned}
\mathcal{U}_{a}^{\star} & =\mathcal{U}_{a}^{n}+\Delta t \dot{\mathcal{U}}_{a}^{n}\left(\mathcal{U}_{a}^{n}, t^{n}\right) ; \\
\mathcal{U}_{a}^{\star \star} & =\mathcal{U}_{a}^{\star}+\Delta t \dot{\mathcal{U}}_{a}^{\star}\left(\mathcal{U}_{a}^{\star}, t^{n+1}\right) ; \\
\mathcal{U}_{a}^{n+1} & =\frac{1}{2}\left(\mathcal{U}_{a}^{n}+\mathcal{U}_{a}^{\star \star}\right) .
\end{aligned}
$$

In comparison with previous work by the authors where the geometry is updated through a standard trapezoidal integration procedure (e.g. $\left.\boldsymbol{x}^{n+1}=\boldsymbol{x}^{n}+\frac{\Delta}{2 \rho_{0}}\left(\boldsymbol{p}^{n}+\boldsymbol{p}^{n+1}\right)\right)$, in this work we resort to the above TVD-RK algorithm [36]. This results in a monolithic time integration procedure where the unknowns $\mathcal{U}=(\boldsymbol{p}, \boldsymbol{F}, \boldsymbol{H}, J, \boldsymbol{x})^{T}$ are all updated through expression (43).

The maximum time step $\Delta t:=t^{n+1}-t^{n}$ is governed by a standard Courant-Friedrichs-Lewy (CFL) condition [68] given as

$$
\Delta t=\alpha_{C F L} \frac{h_{\min }}{c_{p, \max }},
$$

where $c_{p, \max }$ is the maximum $p$-wave speed, $h_{\min }$ is the characteristic particle spacing within the computational domain and $\alpha_{C F L}$ is the CFL stability number. For the numerical computations presented in this paper, unless otherwise stated, a value of $\alpha_{C F L}=0.3$ has been chosen to ensure both accuracy and stability [43].

\section{Discrete angular momentum preserving algorithm}

The resulting JST-SPH algorithm does not intrinsically fulfil conservation of angular momentum, since the strain measures $\{\boldsymbol{F}, \boldsymbol{H}, J\}$ are no longer exclusively obtained from the current geometry [36]. Specifically, the deformation gradient $\boldsymbol{F} \neq \boldsymbol{F}_{\boldsymbol{x}}:=\boldsymbol{\nabla}_{0} \boldsymbol{x}$, its co-factor $\boldsymbol{H} \neq \boldsymbol{H}_{\boldsymbol{x}}:=\frac{1}{2} \boldsymbol{F}_{\boldsymbol{x}} \times \boldsymbol{F}_{\boldsymbol{x}}$ and its Jacobian $J \neq J_{\boldsymbol{x}}:=\operatorname{det} \boldsymbol{F}_{\boldsymbol{x}}$.

In this paper, and following the work of Jibran et al. [36], a monolithic discrete angular momentum projection algorithm is presented. Specifically, the local internal nodal force $\boldsymbol{T}_{a}$ is modified (in a least-square sense) in order to preserve the total angular momentum, whilst still ensuring the global conservation of linear momentum. 
Following Reference [36], sufficient conditions for the global preservation of the discrete linear and angular momentum within a time step are enforced at each stage of the one-step two-stage Runge Kutta time integrator (43) described as:

$$
\sum_{a} V_{a} \boldsymbol{T}_{a}^{\chi}=\mathbf{0} ; \quad \sum_{a} V_{a} \mathscr{X}_{a} \times \boldsymbol{T}_{a}^{\chi}=\mathbf{0} ; \quad \mathscr{X}_{a}=\left\{\begin{array}{cc}
\boldsymbol{x}_{a}^{n}, & \chi=n \\
\boldsymbol{x}_{a}^{n}+\frac{\Delta t}{2 \rho_{0}}\left(\boldsymbol{p}_{a}^{n}+\boldsymbol{p}_{a}^{\star}\right), & \chi=\star
\end{array} ;\right.
$$

where $\chi=\{n, \star\}$.

A least-square minimisation procedure is used to obtain a modified set of internal nodal forces $\hat{\boldsymbol{T}}_{a}$ that satisfy the above conditions (45). This can be achieved by computing the minimum of the following functional [36, 43] (ignoring time arguments for brevity):

$$
\begin{aligned}
\Pi_{\boldsymbol{T}}\left(\hat{\boldsymbol{T}}_{a}, \boldsymbol{\lambda}_{\mathrm{ang}}, \boldsymbol{\lambda}_{\text {lin }}\right) & =\frac{1}{2} \sum_{a} V_{a}\left(\hat{\boldsymbol{T}}_{a}-\boldsymbol{T}_{a}\right) \cdot\left(\hat{\boldsymbol{T}}_{a}-\boldsymbol{T}_{a}\right) \\
& -\boldsymbol{\lambda}_{\mathrm{ang}} \cdot\left(\sum_{a} V_{a} \mathscr{X}_{a} \times \hat{\boldsymbol{T}}_{a}\right)-\boldsymbol{\lambda}_{\operatorname{lin}} \cdot\left(\sum_{a} V_{a} \hat{\boldsymbol{T}}_{a}\right) .
\end{aligned}
$$

After some simple algebra, a modified set of internal nodal forces $\hat{\boldsymbol{T}}_{a}$ arise:

$$
\hat{\boldsymbol{T}}_{a}=\boldsymbol{T}_{a}+\boldsymbol{\lambda}_{\text {ang }} \times \mathscr{X}_{a}+\boldsymbol{\lambda}_{\text {lin }}
$$

The Lagrange multipliers $\left\{\boldsymbol{\lambda}_{\text {ang }}, \boldsymbol{\lambda}_{\text {lin }}\right\}$ are the solutions of the following system of equations

$$
\left[\begin{array}{cc}
\sum_{a} V_{a}\left[\left(\mathscr{X}_{a} \cdot \mathscr{X}_{a}\right) \boldsymbol{I}-\mathscr{X}_{a} \otimes \mathscr{X}_{a}\right] & \sum_{a} V_{a} \hat{\mathscr{X}}_{a} \\
\sum_{a} V_{a} \hat{\mathscr{X}}_{a} & -\sum_{a} V_{a}
\end{array}\right]\left[\begin{array}{c}
\boldsymbol{\lambda}_{\text {ang }} \\
\boldsymbol{\lambda}_{\operatorname{lin}}
\end{array}\right]=\left[\begin{array}{c}
-\sum_{a} V_{a} \mathscr{X}_{a} \times \boldsymbol{T}_{a} \\
\sum_{a} V_{a} \boldsymbol{T}_{a}
\end{array}\right],
$$

with the indicial notation $\left[\hat{\mathscr{X}}_{a}\right]_{i k}=\mathcal{E}_{i j k}\left[\mathscr{X}_{a}\right]_{j}$.

As explained in Section 3.4, the corrected Laplacian employed for the JST stabilisation is not symmetric, leading to the non-conservation of the linear momentum equation (41a). Similar to the method followed in [43], an extra condition can be added for the satisfaction of such requirement, which, together with the global angular momentum preservation, read:

$$
\sum_{a} V_{a} \mathcal{D}\left(\boldsymbol{p}_{a}\right)=\mathbf{0} ; \quad \sum_{a} V_{a} \mathscr{X}_{a} \times \mathcal{D}\left(\boldsymbol{p}_{a}\right)=\mathbf{0} .
$$


Similarly to the least-square minimisation procedure described above, a set of modified (globally conservative) JST dissipation terms can now be obtained by replacing $\left\{\hat{\boldsymbol{T}}_{a}, \boldsymbol{T}_{a}, \boldsymbol{\lambda}_{\text {lin }}, \boldsymbol{\lambda}_{\text {ang }}\right\}$ in (47) and (48) with $\left\{\hat{\mathcal{D}}\left(\boldsymbol{p}_{a}\right), \mathcal{D}\left(\boldsymbol{p}_{a}\right), \boldsymbol{\mu}_{\boldsymbol{D}_{\boldsymbol{p}}}, \boldsymbol{\lambda}_{\boldsymbol{D}_{\boldsymbol{p}}}\right\}$.

Remark 7: To ensure global conservation of the volume map evolution (41d) after the incorporation of the JST stabilisation term, a similar least squared projection is applied for the following functional:

$$
\Pi_{D_{J}}\left(\hat{\mathcal{D}}\left(J_{a}\right), \mu_{D_{J}}\right)=\frac{1}{2} \sum_{a} V_{a}\left(\hat{\mathcal{D}}\left(J_{a}\right)-\mathcal{D}\left(J_{a}\right)\right)^{2}-\mu_{D_{J}} \sum_{a} V_{a} \hat{\mathcal{D}}\left(J_{a}\right),
$$

where $\mu_{D_{J}}$ represents the Lagrange multiplier. By computing the minimum of the above functional (50), the modified JST stabilisation $\hat{\mathcal{D}}\left(J_{a}\right)$ is obtained as

$$
\hat{\mathcal{D}}\left(J_{a}\right)=\mathcal{D}\left(J_{a}\right)+\mu_{D_{J}} ; \quad \mu_{D_{J}}=-\frac{1}{\sum_{a} V_{a}}\left(\sum_{a} V_{a} \mathcal{D}\left(J_{a}\right)\right) .
$$

\section{Algorithmic description}

For ease of understanding, Algorithm 1 summarises the complete algorithmic description of the stabilised Jameson-Schmidt-Turkel Smooth Particle Hydrodynamics (JST-SPH) mixed-based $\{\boldsymbol{p}, \boldsymbol{F}, \boldsymbol{H}, J\}$ methodology with all the necessary numerical ingredients ${ }^{5}$. Notice that simpler $\{\boldsymbol{p}, \boldsymbol{F}\},\{\boldsymbol{p}, \boldsymbol{F}, J\}$ and $\{\boldsymbol{p}, J\}$ versions of the algorithm can be easily obtained by neglecting the relevant geometric conservation laws (i.e. (41c) and/or (41d)).

\section{Numerical examples}

In this section, a series of challenging numerical examples are presented in order to assess the robustness, effectiveness and applicability of the proposed framework described above (see Algorithm 1). Examples presented focus on (reversible) hyperelastic constitutive models so that no physical dissipation is present in the problem (maybe helping with the stability of the problem).

\footnotetext{
${ }^{5}$ For completeness, the error analysis of the overall JST-SPH framework on a one dimensional linear advection equation is shown in Appendix C.
} 


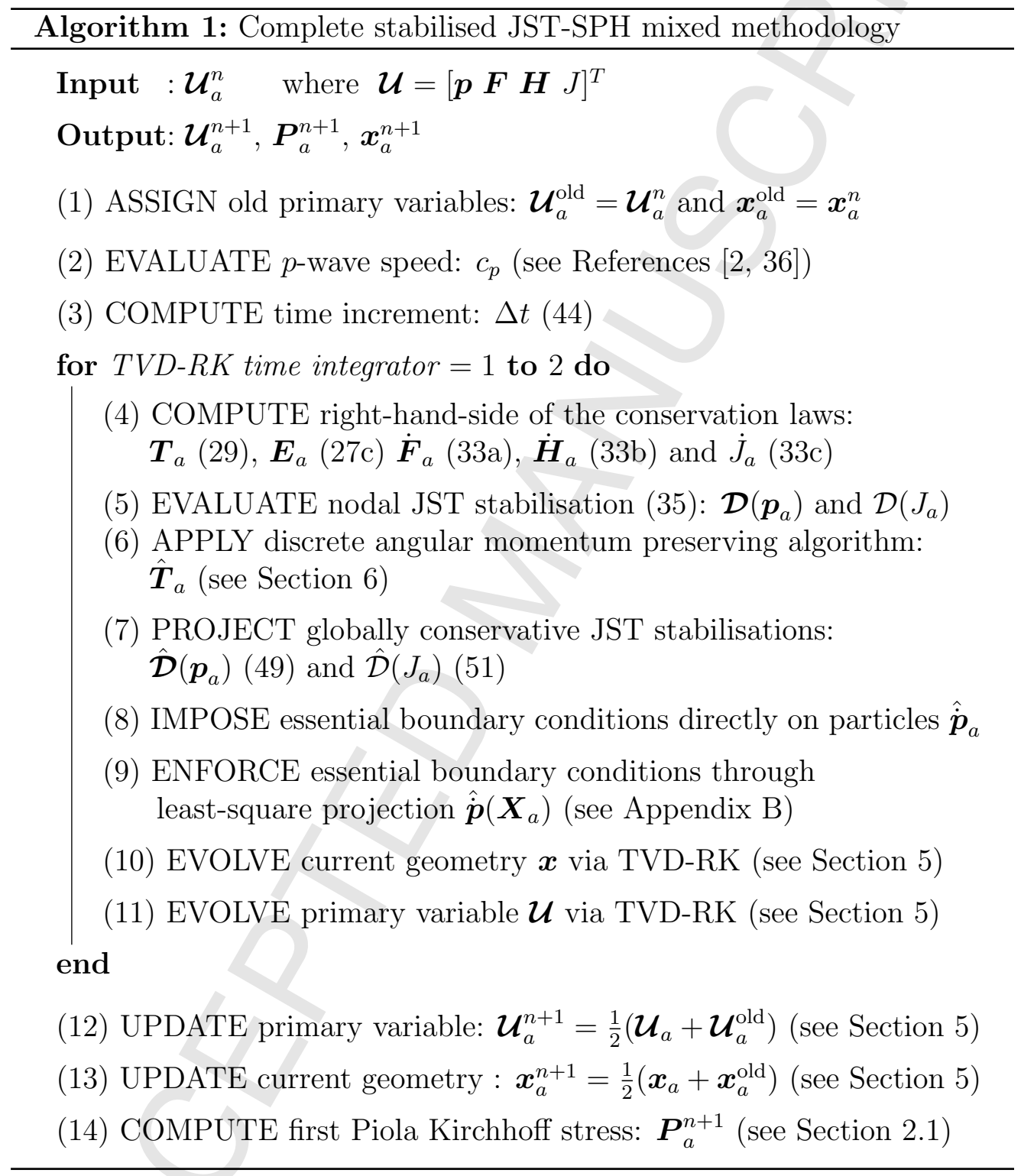


Three stabilised JST-SPH methodologies are analysed, namely $\{\boldsymbol{p}, \boldsymbol{F}\}$ JST-SPH, $\{\boldsymbol{p}, \boldsymbol{F}, J\}$ JST-SPH and $\{\boldsymbol{p}, \boldsymbol{F}, \boldsymbol{H}, J\}$ JST-SPH. For comparison purposes, some of the results are benchmarked against the well known nonLadyzhenskaya-Babuska-Brezzi (non-LBB) compliant B-bar hexadedral element [8] and the LBB compliant Q2-Q1 hexahedral element (i.e. Taylor-Hood element [69]).

We also compare against the following Finite Volume based methodologies: $\{\boldsymbol{p}, \boldsymbol{F}\}$ Upwind Vertex Centred (Upwind-VCFVM) [44], $\{\boldsymbol{p}, \boldsymbol{F}\}$ JamesonSchmidt-Turkel Vertex Centred (JST-VCFVM) [43], $\{\boldsymbol{p}, \boldsymbol{F}\}$ Upwind Cell Centred (i.e. TOUCH [36, 37]) and $\{\boldsymbol{p}, \boldsymbol{F}\}$ Hyperelastic-GLACE [47]. In addition, we compare against the following Finite Element based tetrahedral elements: $\{\boldsymbol{p}, \boldsymbol{F}, J\}$ Petrov-Galerkin Finite Element Method (PG-FEM) [42] and the LBB compliant Hu-Washizu $\left\{\boldsymbol{v}, \boldsymbol{F}, \boldsymbol{H}, J, \boldsymbol{\Sigma}_{\boldsymbol{F}}, \boldsymbol{\Sigma}_{\boldsymbol{H}}, \Sigma_{J}\right\}$ mixed-based Finite Element method (see [49] for further details).

\subsection{Cable}

A simple elastic wave propagation problem is presented. This problem was first studied in Reference [70] and later explored in [17, 18]. A rod of dimensions $10 \mathrm{~m} \times 0.2 \mathrm{~m} \times 0.2 \mathrm{~m}$ is fixed on the left end and set free on the right end. Symmetric boundary conditions (i.e. roller support) are enforced on the rest of the boundaries. An initial velocity $v_{0}$ of $5 \mathrm{~m} / \mathrm{s}$ is imposed on the left quarter of the rod considering a discretisation of $100 \times 3 \times 3$ particles uniformly distributed. A linear elastic material of density $\rho_{0}=8000 \mathrm{~kg} / \mathrm{m}^{3}$, Youngs's modulus $E=200 \mathrm{GPa}$ and Poisson's ratio $\nu=0$ is used. In this particular case, an analytical solution can be derived [37] which will be used for comparison purposes.

As pointed out in References $[17,18,70]$, any reported attempts to eliminate the so-called tensile instability mechanisms [70] using either Eulerian SPH or Updated Lagrangian Corrected Smooth Particle Hydrodynamics (CSPH) have been unsuccessful. In contrast, the authors in [18] have shown that this problem can be solved by resorting to a Total Lagrangian formalism. However, the methodology proposed in that Reference still suffers from excessive oscillation due to insufficient stabilisation. The main objective of this example is to show that the proposed $\{\boldsymbol{p}, \boldsymbol{F}\}$ JST-SPH methodology alleviates the appearance of spurious mechanisms in the vicinity of sharp spatial gradients.

Figure 3 shows the time history of both horizontal velocity and displacement at the right tip end of the $\operatorname{rod}\left(\boldsymbol{X}=[10,0.2,0.2]^{T} \mathrm{~m}\right)$. These results 
are benchmarked against the analytical solution [37]. The proposed $\{\boldsymbol{p}, \boldsymbol{F}\}$ JST-SPH framework predicts the correct arrival time of the velocity shock. However, over- and under-shoots oscillatory behaviour can be seen when no second order harmonic dissipation is considered (i.e. $\varepsilon_{\boldsymbol{p}}^{(2)}=0$ ). This unwanted behaviour is removed once this term is activated.

Figure 4 shows the spatial pressure distribution throughout the whole domain at time $t=0.006 \mathrm{~s}$ using three different values of $\varepsilon_{\boldsymbol{p}}^{(2)}$, namely $\varepsilon_{\boldsymbol{p}}^{(2)}=$ $\left\{0, \frac{1}{32}, \frac{1}{8}\right\}$. Clearly, the oscillatory behaviour is observed when using the value of $\varepsilon_{\boldsymbol{p}}^{(2)}=0$ (see Figure $4 \mathrm{a}$ ). This spurious pressure pattern is removed after progressive activation of $\varepsilon_{\boldsymbol{p}}^{(2)}$ (see Figures $4 \mathrm{~b}$ and $4 \mathrm{c}$ ).

\subsection{Swinging cube}

As presented in References $[1,2,36,37,41-44,71]$ this example (see Figure 5) shows a cube of unit side length with symmetric boundary conditions (e.g. roller supports) at faces $X=0, Y=0$ and $Z=0$ and skewsymmetric boundary conditions (i.e. restricted tangential displacement) at faces $X=1 \mathrm{~m}, Y=1 \mathrm{~m}$ and $Z=1 \mathrm{~m}$. When small deformations are considered, this example has a closed-form solution for the displacement field described as

$$
\boldsymbol{u}(\boldsymbol{X}, t)=U_{0} \cos \left(\frac{\sqrt{3}}{2} c_{d} \pi t\right)\left[\begin{array}{l}
A \sin \left(\frac{\pi X_{1}}{2}\right) \cos \left(\frac{\pi X_{2}}{2}\right) \cos \left(\frac{\pi X_{3}}{2}\right) \\
B \cos \left(\frac{\pi X_{1}}{2}\right) \sin \left(\frac{\pi X_{2}}{2}\right) \cos \left(\frac{\pi X_{3}}{2}\right) \\
C \cos \left(\frac{\pi X_{1}}{2}\right) \cos \left(\frac{\pi X_{2}}{2}\right) \sin \left(\frac{\pi X_{3}}{2}\right)
\end{array}\right]
$$

with $c_{d}=\sqrt{\frac{\lambda+2 \mu}{\rho_{0}}}$. Parameters $\{A, B, C\}$ are constants chosen such that $A=$ $B=C$ to ensure the existence of a non-zero pressure field. For values of $U_{0}$ below $0.001 \mathrm{~m}$, the solution can be regarded to be linear and the closed-form expression (52) holds. The problem is initialised with a given deformation gradient $\boldsymbol{F}(\boldsymbol{X}, 0)=\boldsymbol{I}+\boldsymbol{\nabla}_{0} \boldsymbol{u}$ where the terms $\boldsymbol{\nabla}_{0} \boldsymbol{u}$ are evaluated from the above expression (52). In particular, we select a value of $U_{0}=5 \times 10^{-4} \mathrm{~m}$ and $A=B=C=1$. A linear elastic material is chosen with Young's modulus $E=17 \mathrm{MPa}$, material density $\rho_{0}=1100 \mathrm{~kg} / \mathrm{m}^{3}$ and Poisson's ratio $\nu=0.3$.

The main aim of this example is to show the convergence behaviour of the proposed $\{\boldsymbol{p}, \boldsymbol{F}\}$ JST-SPH in a three dimensional setting. In this paper, the convergence analysis is carried out by measuring $L_{2}$ norm errors between the numerical and analytical solution. To ensure accuracy in the computation of this norm, the error is evaluated by means of a sufficiently fine 


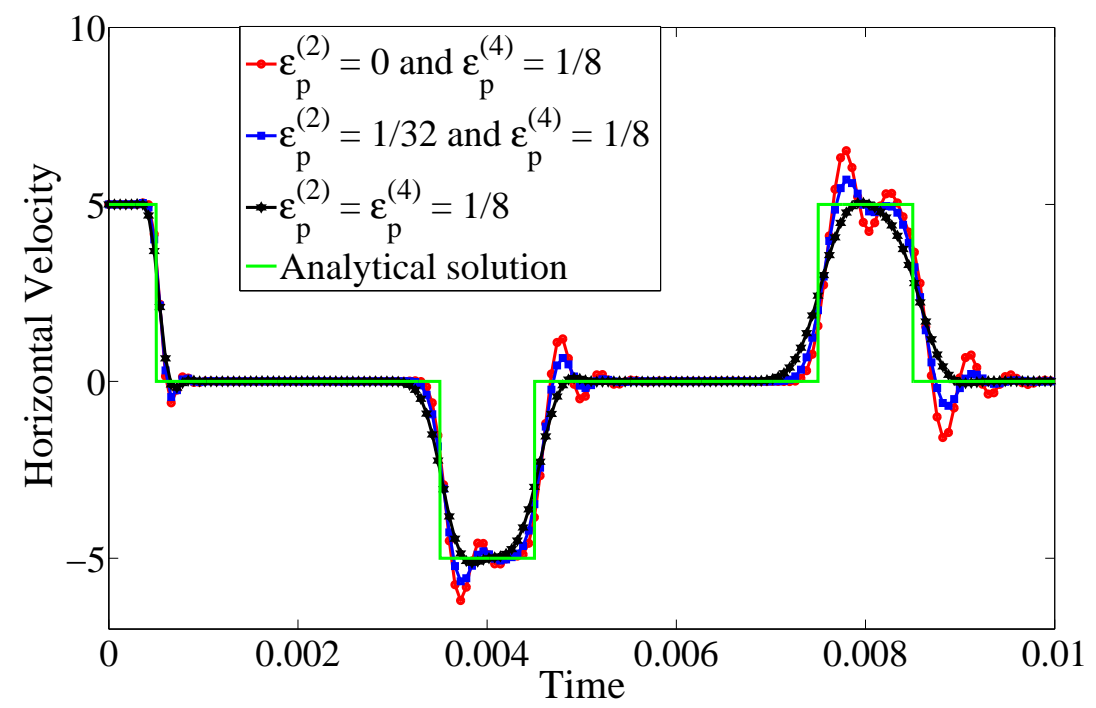

(a) Horizontal velocity

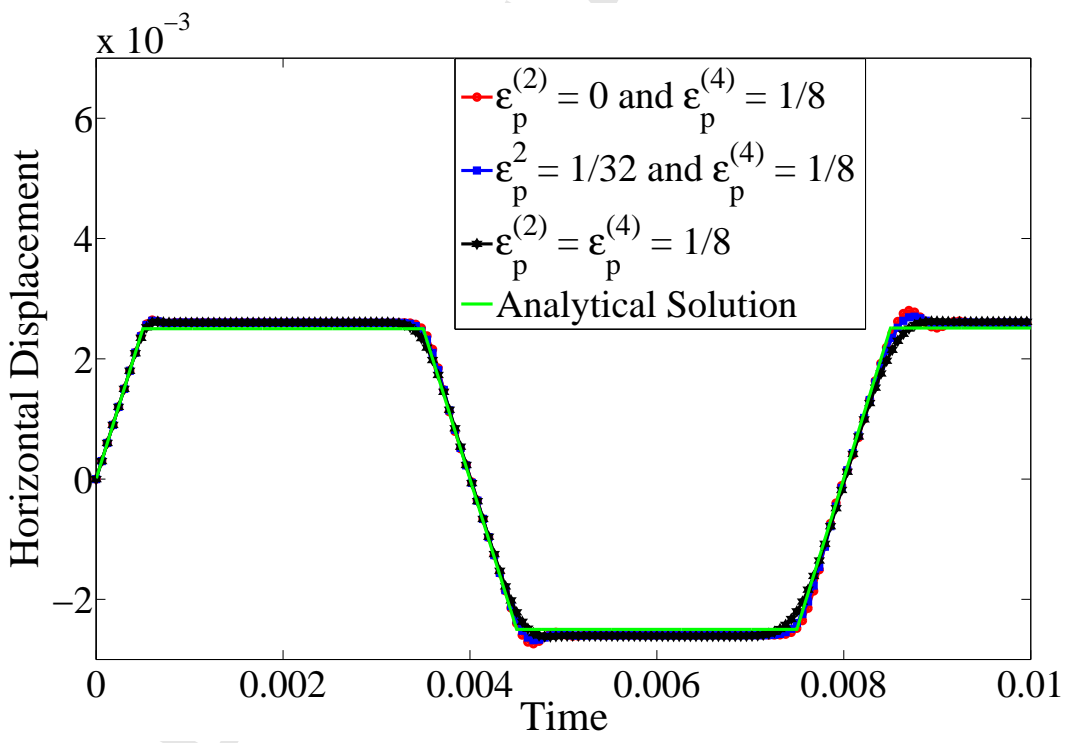

(b) Horizontal displacement

Figure 3: Cable: Time evolution of the (a) Horizontal velocity; and (b) Horizontal displacement at position $\boldsymbol{X}=[10,0.2,0.2]^{T}$. Results are obtained using the proposed $\{\boldsymbol{p}, \boldsymbol{F}\}$ JST-SPH. A linear elastic material is used with density $\rho_{0}=8000 \mathrm{~kg} / \mathrm{m}^{3}$, Young's modulus $E=200 \mathrm{GPa}$, Poisson's ratio $\nu=0$ and $\alpha_{C F L}=0.3$. Discretisation of $100 \times 3 \times 3$ material particles. 
$t=0.006 \mathrm{~s}$

(a) JST parameters used: $\varepsilon_{\boldsymbol{p}}^{(4)}=\frac{1}{8}$ and $\varepsilon_{\boldsymbol{p}}^{(2)}=0$

(b) JST parameters used: $\varepsilon_{\boldsymbol{p}}^{(4)}=\frac{1}{8}$ and $\varepsilon_{\boldsymbol{p}}^{(2)}=\frac{1}{32}$

(c) JST parameters used: $\varepsilon_{\boldsymbol{p}}^{(4)}=\frac{1}{8}$ and $\varepsilon_{\boldsymbol{p}}^{(2)}=\frac{1}{8}$

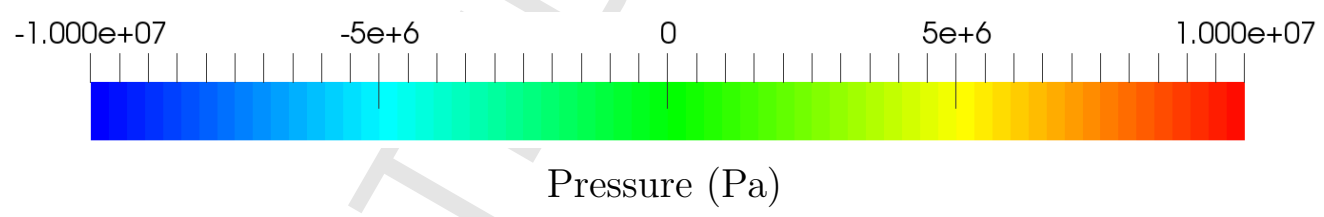

Figure 4: Cable: Time evolution of the deformation plotted with pressure distribution at time $t=0.006 \mathrm{~s}$ using the mixed-based $\{\boldsymbol{p}, \boldsymbol{F}\}$ JST-SPH algorithm with (a) $\varepsilon_{\boldsymbol{p}}^{(2)}=0$; (b) $\varepsilon_{\boldsymbol{p}}^{(2)}=\frac{1}{32}$; (c) $\varepsilon_{\boldsymbol{p}}^{(2)}=\frac{1}{8}$. A neo-Hookean material is used with density $\rho_{0}=8000 \mathrm{~kg} / \mathrm{m}^{3}$, Young's modulus $E=200$ GPa, Poisson's ratio $\nu=0$ and $\alpha_{C F L}=0.3$. Discretisation of $100 \times 3 \times 3$ material particles. 


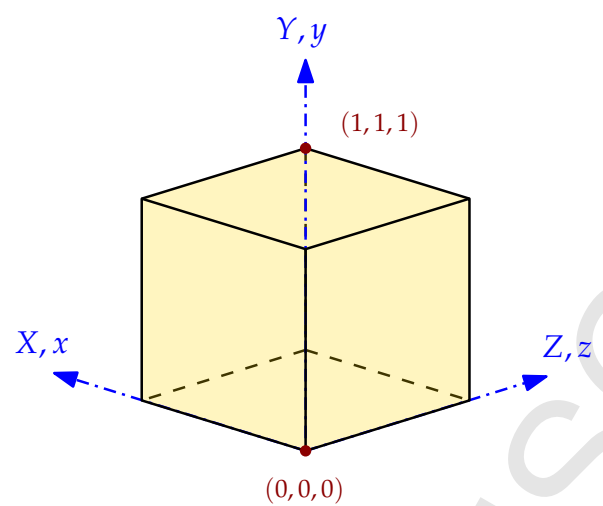

Figure 5: Swinging cube configuration

background cloud of quadrature points. Figure 6 depicts the expected second order $L_{2}$ norm convergence pattern for both linear momentum and the components of the stress tensor. It is interesting to note that the truncation error of the algorithm is not adversely affected even without imposing the exact enforcement of essential boundary conditions. For this reason, and for computational efficiency, exact enforcement of essential boundary conditions (see Appendix B) is usually disregarded in SPH methods [17-19].

\subsection{Spinning cube}

Similar to the "water bubble" problem presented in Reference [52] (see Section 5.2 on pg. 108), we consider a cube of unit side length is left free on all its sides $[36,37]$. The cube is subjected to an initial constant angular velocity $\boldsymbol{\omega}_{0}=[0,0,105]^{T} \mathrm{rad} / \mathrm{s}$ relative to its centroid and is then left rotating in space. A nearly incompressible neo-Hookean material is utilised with the following material properties: density $\rho_{0}=1100 \mathrm{~kg} / \mathrm{m}^{3}$, Young's modulus $E=17 \mathrm{MPa}$ and Poisson's ratio $\nu=0.3$. The main objective of this example is to illustrate the capability of the $\{\boldsymbol{p}, \boldsymbol{F}\}$ JST-SPH framework (with a discretisation of $6 \times 6 \times 6$ particles) to globally preserve the linear and angular momentum. Figure 7 a shows the time evolution of the components of the total angular momentum. As it should be expected, the block exhibits no change in angular momentum when activating the discrete momentum projection algorithm (see Section 6). Figure 7b depicts the global conservation of linear momentum, which is zero to machine accuracy. For visualisation purposes, Figure 8 shows the time evolution of the deformation of the block with a very smooth pressure distribution. 


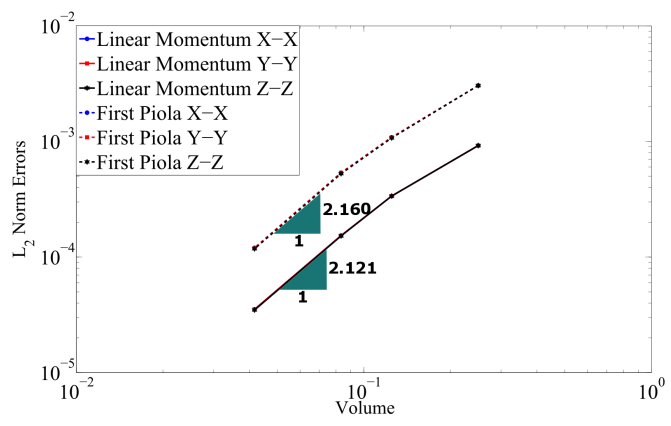

(a) With boundary projection

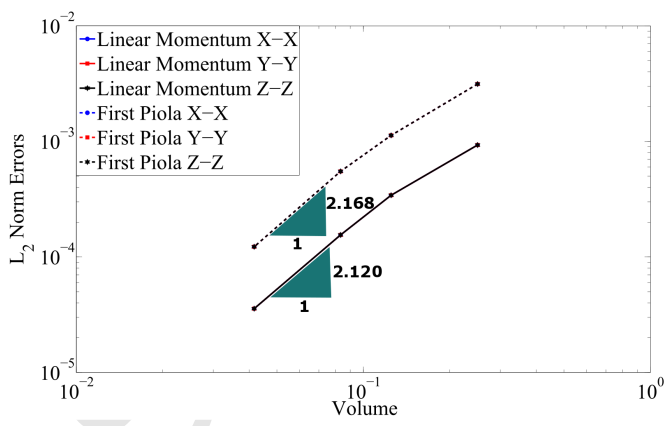

(b) Without boundary projection

Figure 6: Swinging cube: $L_{2}$ norm convergence of the components of both linear momentum and stresses using: (a) With Lagrange multiplier least-square projection; and (b) Without Lagrange multiplier least-square projection. Results are obtained using the proposed $\{\boldsymbol{p}, \boldsymbol{F}\}$ JST-SPH. A neo-Hookean material is used with density $\rho_{0}=1100 \mathrm{~kg} / \mathrm{m}^{3}$, Young's modulus $E=17 \mathrm{MPa}$, Poisson's ratio $\nu=0.3$ and $\alpha_{C F L}=0.3$. JST parameter used: $\varepsilon_{\boldsymbol{p}}^{(2)}=0$ and $\varepsilon_{\boldsymbol{p}}^{(4)}=\frac{1}{8}$.

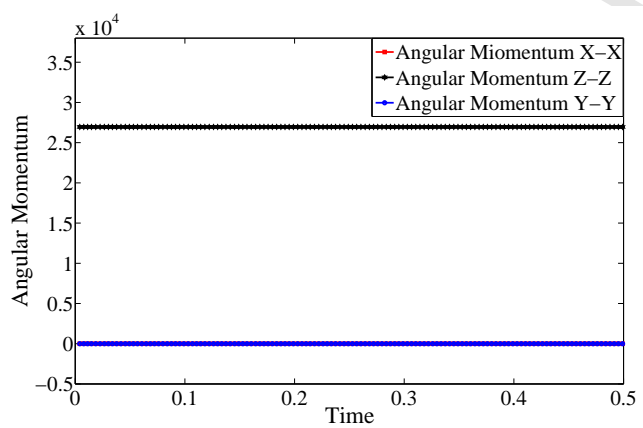

(a) Angular Momentum

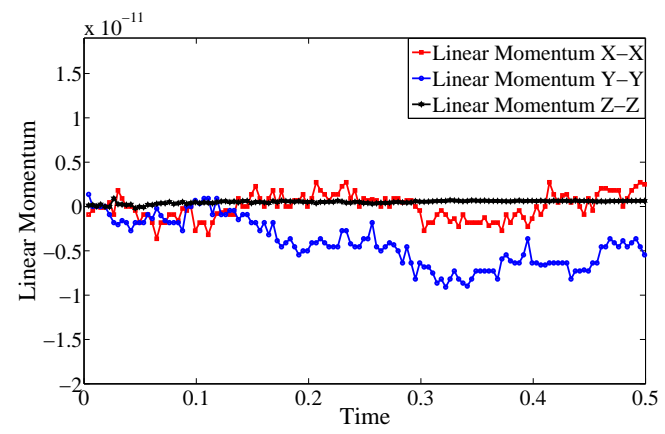

(b) Linear Momentum

Figure 7: Spinning cube: Time evolution of the components of (a) Angular momentum; and (b) Linear momentum. Results are obtained using the proposed $\{\boldsymbol{p}, \boldsymbol{F}\}$ JST-SPH with the consideration of the angular momentun projection algorithm. A neo-Hookean material is used with density $\rho_{0}=1100 \mathrm{~kg} / \mathrm{m}^{3}$, Young's modulus $E=17 \mathrm{MPa}$, Poisson's ratio $\nu=0.3$ and $\alpha_{C F L}=0.3$. Discretisation of $6 \times 6 \times 6$ material particles. JST parameter used: $\varepsilon_{\boldsymbol{p}}^{(2)}=0$ and $\varepsilon_{\boldsymbol{p}}^{(4)}=\frac{1}{8}$. 
$t=0.0148 \mathrm{~s}$

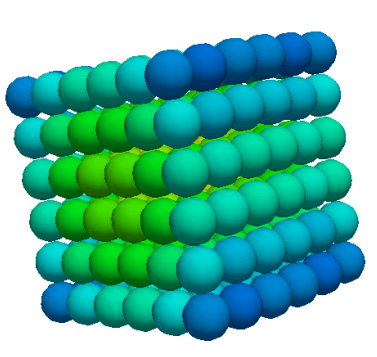

$t=0.0494 \mathrm{~s}$

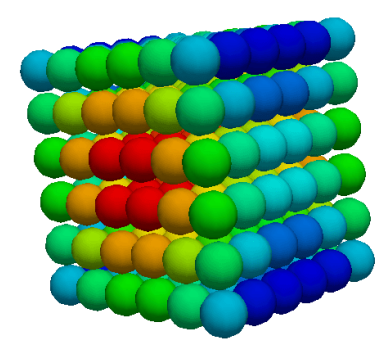

$t=0.0839 \mathrm{~s}$

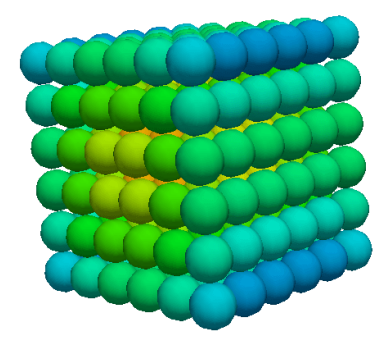

$0.000 e+00$ $t=0.0266 \mathrm{~s}$

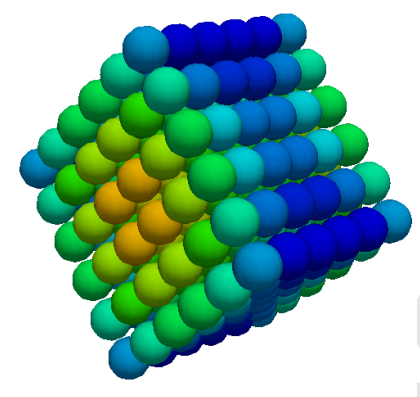

$$
t=0.0608 \mathrm{~s}
$$

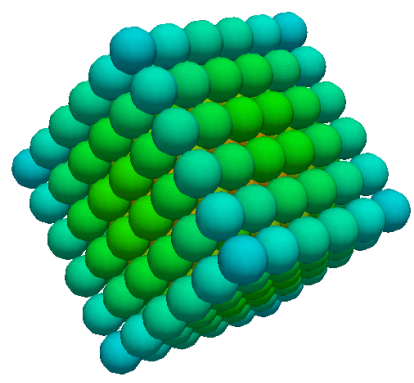

$$
t=0.095 \mathrm{~s}
$$
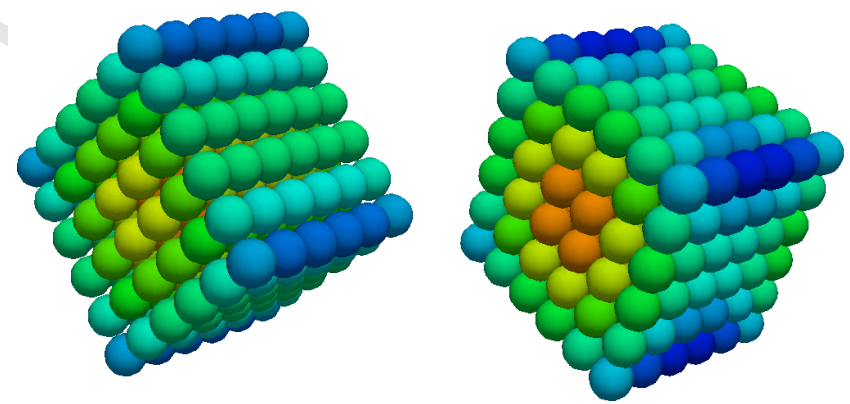

$7.5 e+5$

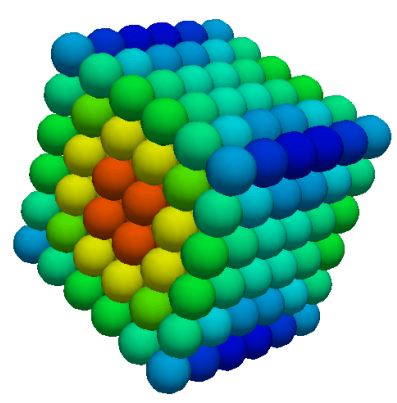

$t=0.107 \mathrm{~s}$

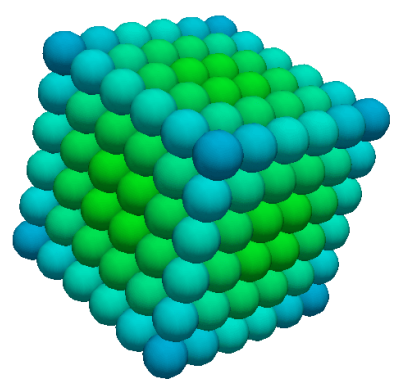

$$
t=0.0722 \mathrm{~s}
$$

$1.500 e+06$

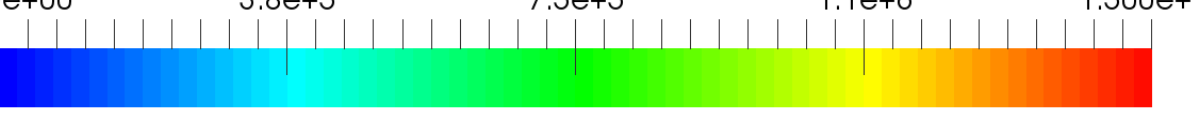

\section{Pressure $(\mathrm{Pa})$}

30

Figure 8: Spinning cube: Time evolution of the deformation plotted with pressure distribution using the mixed-based $\{\boldsymbol{p}, \boldsymbol{F}\}$ JST-SPH. A neoHookean material is used with density $\rho_{0}=1100 \mathrm{~kg} / \mathrm{m}^{3}$, Young's modulus $E=17 \mathrm{MPa}$, Poisson's ratio $\nu=0.3$ and $\alpha_{C F L}=0.3$. Discretisation of $6 \times 6 \times 6$ material particles. JST parameters used: $\varepsilon_{\boldsymbol{p}}^{(2)}=0$ and $\varepsilon_{\boldsymbol{p}}^{(4)}=\frac{1}{8}$. 


\subsection{Tensile cube}

A unit cube clamped at the bottom and subjected to traction-free conditions on the rest of the boundaries is subjected to a linear variation in initial velocity field $\boldsymbol{v}_{0}=\left(0, \frac{V Y}{L}, 0\right)^{T}$ (where $V=800 \mathrm{~m} / \mathrm{s}$ ). The objective of this example $[1,42]$ is to illustrate the performance of the stabilised $\{\boldsymbol{p}, \boldsymbol{F}\}$ JSTSPH formulation discretised with $8 \times 8 \times 8$ particles, with and without the exact enforcement of essential boundary conditions (see Appendix B). This problem is solved using a nearly incompressible neo-Hookean material with Young's modulus $E=21 \mathrm{GPa}$, density $\rho_{0}=7 \mathrm{Mg} / \mathrm{m}^{3}$ and Poisson's ratio $\nu=0.3$.

Comparison of a sequence of deformed states simulated using the $\{\boldsymbol{p}, \boldsymbol{F}\}$ JST-SPH algorithm (with and without the imposition of essential boundary conditions) is shown in Figure 9. For further comparison, the displacement evolution for a boundary material particle initially placed at position $\boldsymbol{X}_{B}=$ $[0,0,0]^{T}$ is also monitored (see Figure 10). It is clear that null displacements $\boldsymbol{u}$ can be obtained with the exact enforcement of homogeneous Dirichlet conditions $\boldsymbol{u}\left(\boldsymbol{X}_{B}\right)=\mathbf{0}$, whereas a very small magnitude can be observed by enforcing zero values directly on the relevant particle $\boldsymbol{u}_{B}=\mathbf{0}$. Both approaches show very good agreement in terms of pressure and also the deformations (see Figure 9). For this reason, and as reported in References $[17,19]$, it is often preferred to use the latter approach due to computational efficiency.

For completeness, the exact same problem is also solved using three alternative approaches, namely the classical displacement-based SPH [17], $\{\boldsymbol{p}, \boldsymbol{F}, J\}$ JST-SPH and $\{\boldsymbol{p}, \boldsymbol{F}, \boldsymbol{H}, J\}$ JST-SPH. As shown in Figure 11a, the standard displacement-based SPH exhibits spurious modes in the solution. This shortcoming can be alleviated using the proposed JST-SPH frameworks (see Figures 9a, 11b and 11c). Specifically, only one JST parameter is required for the simulation of this problem, that is $\varepsilon_{\boldsymbol{p}}^{(4)}=1 / 8$. It is worth highlighting that all proposed JST-SPH frameworks provide practically identical results.

\subsection{Symmetric coining}

A rigid punch test is presented in $[17,58]$ and illustrated in Figure 12. Given the symmetries of the problem, only one quarter of the domain (0.03 $\mathrm{m} \times 0.01 \mathrm{~m} \times 0.005 \mathrm{~m})$ is discretised. The material is compressed to $54 \%$ of its original height based on the application of a boundary velocity field 
$t=0.00058 \mathrm{~s}$

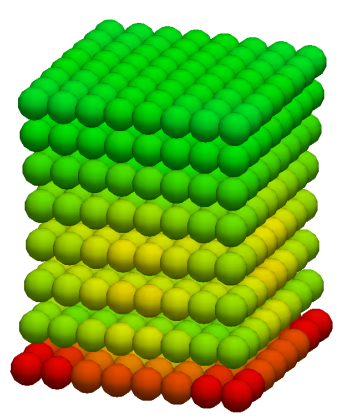

$t=0.0017 \mathrm{~s}$

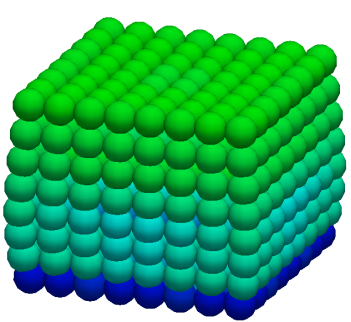

$t=0.0021 \mathrm{~s}$

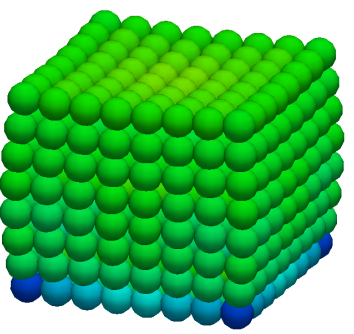

(a) With Lagrange multiplier boundary projecton
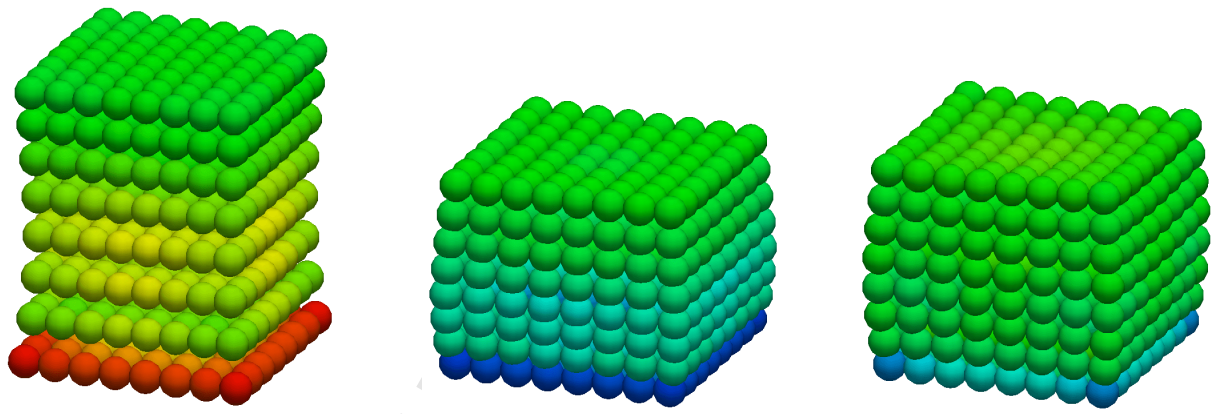

(b) Without Lagrange multiplier boundary projecton

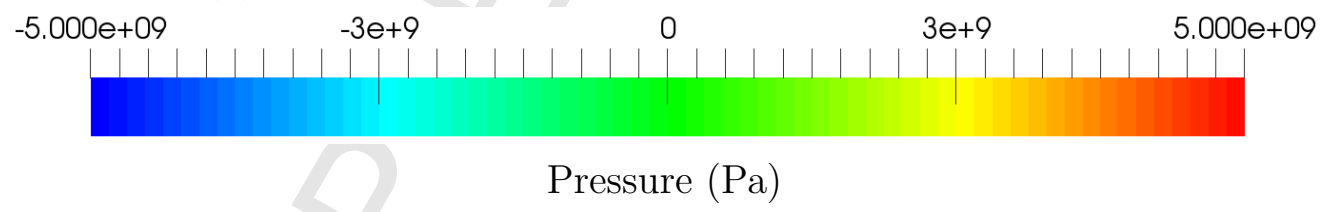

Figure 9: Tensile cube: Results obtained using the mixed-based $\{\boldsymbol{p}, \boldsymbol{F}\}$ JSTSPH algorithm: (a) With the exact enforcement of essential boundary conditions; and (b) Without the exact enforcement of essential boundary conditions. A neo-Hookean material is used with density $\rho_{0}=1100 \mathrm{~kg} / \mathrm{m}^{3}$, Young's modulus $E=17 \mathrm{MPa}$, Poisson's ratio $\nu=0.3$ and $\alpha_{\mathrm{CFL}}=0.3$. Discretisation of $8 \times 8 \times 8$ particles. JST parameters used: $\varepsilon_{\boldsymbol{p}}^{(2)}=0$ and $\varepsilon_{\boldsymbol{p}}^{(4)}=\frac{1}{8}$. 


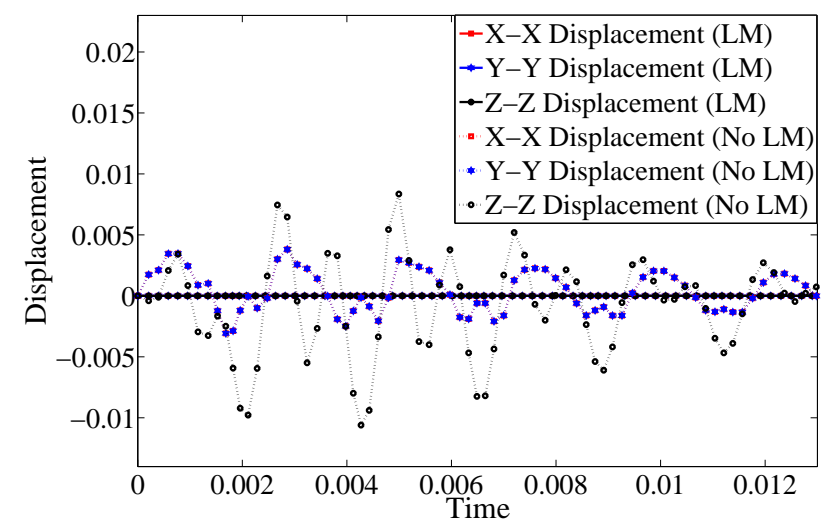

Figure 10: Tensile cube: Time evolution of the components of the displacement field at boundary position $\boldsymbol{X}_{B}=[0,0,0]^{T}$ using the mixed-based $\{\boldsymbol{p}, \boldsymbol{F}\}$ JST-SPH via (a) Lagrange Multiplier (LM) boundary projection; and (b) Without Lagrange Multiplier (No LM) boundary projection. A neo-Hookean material is used with density $\rho_{0}=1100 \mathrm{~kg} / \mathrm{m}^{3}$, Young's modulus $E=17$ $\mathrm{MPa}$, Poisson's ratio $\nu=0.3$ and $\alpha_{\mathrm{CFL}}=0.3$. Discretisation of $8 \times 8 \times 8$ particles. JST parameters used: $\varepsilon_{\boldsymbol{p}}^{(2)}=0$ and $\varepsilon_{\boldsymbol{p}}^{(4)}=\frac{1}{8}$. 


$$
t=0.00058 \mathrm{~s} \quad t=0.0017 \mathrm{~s} \quad t=0.0021 \mathrm{~s}
$$
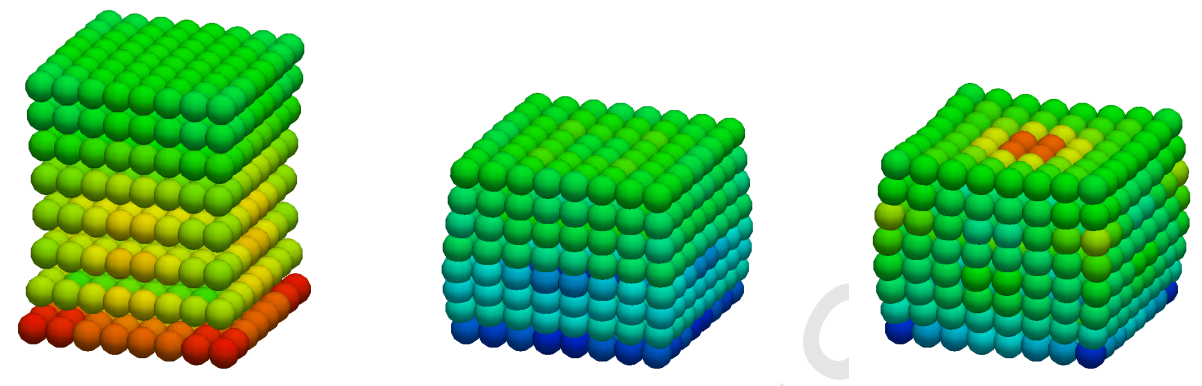

(a) Classical displacement-based SPH
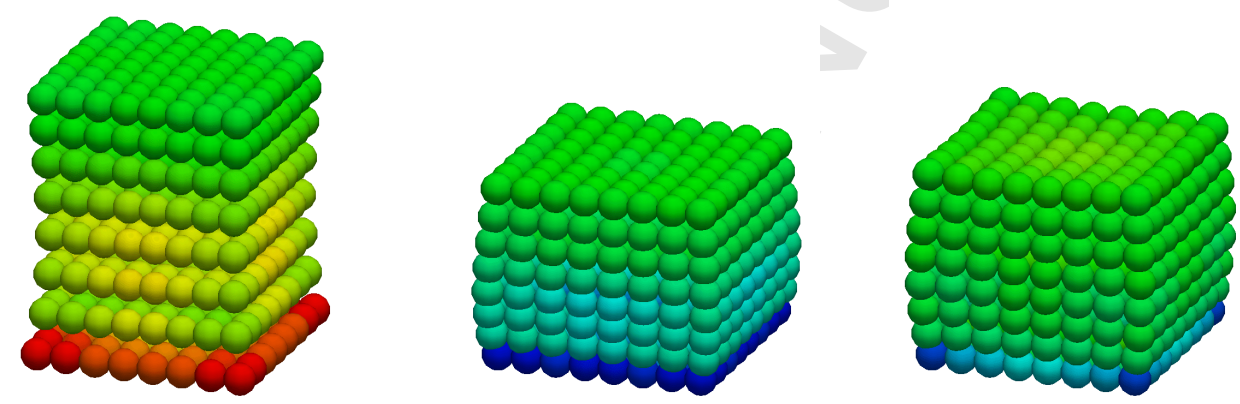

(b) Mixed-based $\{\boldsymbol{p}, \boldsymbol{F}, J\}$ JST-SPH $\left(\varepsilon_{\boldsymbol{p}}^{(2)}=\varepsilon_{J}^{(2)}=\varepsilon_{J}^{(4)}=0\right.$ and $\left.\varepsilon_{\boldsymbol{p}}^{(4)}=\frac{1}{8}\right)$
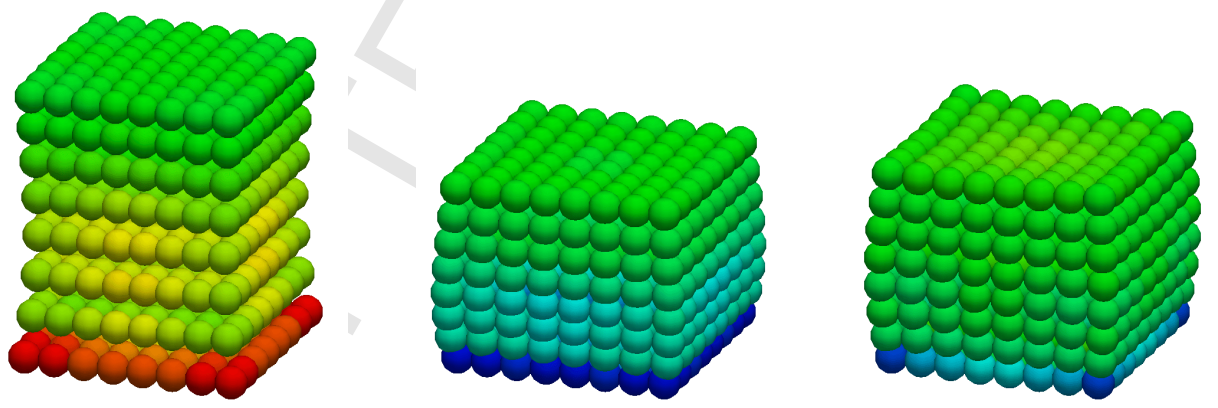

(c) Mixed-based $\{\boldsymbol{p}, \boldsymbol{F}, \boldsymbol{H}, J\}$ JST-SPH $\left(\varepsilon_{\boldsymbol{p}}^{(2)}=\varepsilon_{J}^{(2)}=\varepsilon_{J}^{(4)}=0\right.$ and $\left.\varepsilon_{\boldsymbol{p}}^{(4)}=\frac{1}{8}\right)$ $-5.000 e+09$
$L$

\section{Pressure (Pa)}

Figure 11: Tensile cube: Results obtaifed using: (a) Classical displacementbased SPH; (b) Mixed-based $\{\boldsymbol{p}, \boldsymbol{F}, J\}$ JST-SPH; and (c) Mixed-based $\{\boldsymbol{p}, \boldsymbol{F}, \boldsymbol{H}, J\}$ JST-SPH. A neo-Hookean material is used with density $\rho_{0}=$ $1100 \mathrm{~kg} / \mathrm{m}^{3}$, Young's modulus $E=17 \mathrm{MPa}$, Poisson's ratio $\nu=0.3$ and $\alpha_{\mathrm{CFL}}=0.3$. Discretisation of $8 \times 8 \times 8$ particles. 


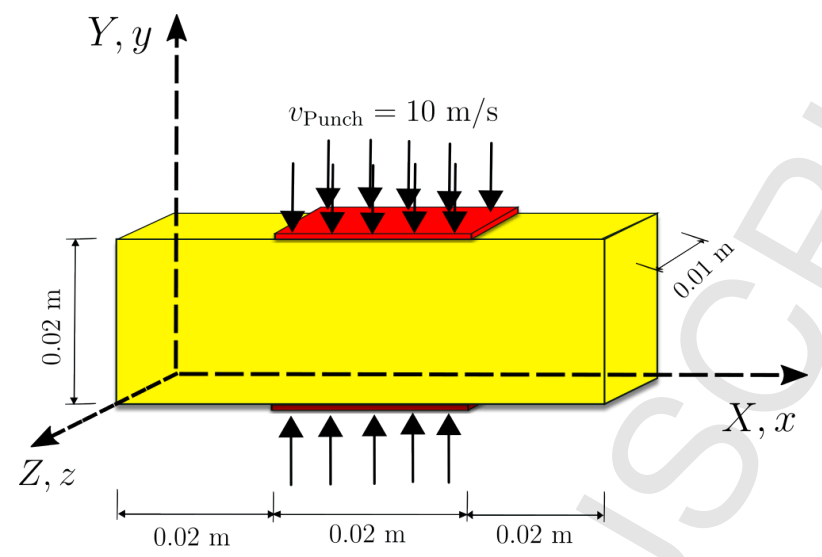

Figure 12: Symmetric coining configuration

$\boldsymbol{v}_{0}=(0,-10,0)^{T} \mathrm{~m} / \mathrm{s}$ applied on one-third of top side $\boldsymbol{X}_{0}$ described as:

$$
\boldsymbol{X}_{0}=\left[\begin{array}{c}
0.01 \geq X \geq 0 \\
Y=0.01 \\
0.005 \geq Z \geq 0
\end{array}\right] \mathrm{m} .
$$

In this particular case, Reference [17] (Section 2.4.3, pg. 2696-2697) shows that the Total Lagrangian CSPH algorithm fails to capture the extremely large distortion for a ratio of the bulk and shear moduli of $\kappa / \mu \approx 5$. To rectify this shortcoming, Vidal et al. [17] developed a stabilised Updated Lagrangian CSPH algorithm via the use of a Hessian operator. However, this Updated Lagrangian CSPH algorithm is relatively expensive since it requires the computation of a third-order tensor and frequent updates of the reference configuration.

The main objective of this example is to show the robustness of the Total Lagrangian mixed-based $\{\boldsymbol{p}, \boldsymbol{F}\}$ JST-SPH. More interestingly, we use a relatively large value of Poisson's ratio $\nu=0.495(\kappa / \mu \approx 100)$ to represent the near incompressibility regime. A nearly incompressible neo-Hookean model is used with material density $\rho_{0}=1000 \mathrm{~kg} / \mathrm{m}^{3}$ and Young's modulus $E=1$ MPa. For comparison purposes, the $\{\boldsymbol{p}, \boldsymbol{F}\}$ SPH (i.e. no stabilisation), $\{\boldsymbol{p}, \boldsymbol{F}\}$ JST-SPH, $\{\boldsymbol{p}, \boldsymbol{F}, J\}$ JST-SPH and $\{\boldsymbol{p}, \boldsymbol{F}, \boldsymbol{H}, J\}$ JST-SPH methodologies will be analysed.

Comparison of a sequence of snapshots using the above four methodologies (with a discretisation of $31 \times 11 \times 6$ particles) is shown in Figure 13 . It is clear that the unstabilised $\{\boldsymbol{p}, \boldsymbol{F}\}$ SPH methodology (see Figure 13a) 
introduces spurious pressure oscillations. These non-physical pressure instabilities can be efficiently removed by using the alternative stabilised schemes (see Figures 13b, 13c, 13d), with the use of only one JST biharmonic stabilising term $\varepsilon_{\boldsymbol{p}}^{(4)}=\frac{1}{8}$. Very good agreement for both pressure and deformations can be observed for all proposed JST-SPH methodologies. For visualisation purposes, Figure 14 illustrates the time evolution of the deformation of the problem simulated using $\{\boldsymbol{p}, \boldsymbol{F}\}$ JST-SPH, displaying a smooth pressure contour.

\subsection{Nearly incompressible punch problem}

This nearly incompressible punch example was first studied in Reference [67] within the context of quasi-static mesh free methods. In this Reference, a mesh free algorithm is combined with computations in an ad-hoc background mesh in order to prevent the appearance of spurious oscillations in the near incompressibility limit. However, it is not straightforward to adapt this methodology to three-dimensional applications.

The main objective of this example is to show the ability of the proposed methodology to suppress severe pressure oscillations in highly constrained problems. A block of $1 \mathrm{~m} \times 0.5 \mathrm{~m} \times 0.1 \mathrm{~m}$ is left free on its top face and constrained with rollers (i.e. symmetric boundary conditions) on the rest of the boundaries (see Figure 15). The block is compressed up to a total of $28 \%$ of its original height via a uniform boundary velocity field $\boldsymbol{v}_{0}=$ $(0,-10,0)^{T} \mathrm{~m} / \mathrm{s}$ applied on the mid portion of the top face $\boldsymbol{X}_{0}$ described as:

$$
\boldsymbol{X}_{0}=\left[\begin{array}{c}
\frac{2}{3} \geq X \geq \frac{1}{3} \\
Y=0.5 \\
0.1 \geq Z \geq 0
\end{array}\right] \mathrm{m}
$$

A nearly incompressible neo-Hookean material is used where the density $\rho_{0}=1000 \mathrm{~kg} / \mathrm{m}^{3}$, Young's Modulus $E=1 \mathrm{MPa}$ and Poisson's ratio $\nu=0.499$ (i.e. incompressibility limit $\frac{\kappa}{\mu} \approx 500$ ).

For benchmarking purposes, four different methodologies with a discretisation of $21 \times 11 \times 3$ particles are used. As it is well known, classical displacement-based SPH shows excessive pressure fluctuations which eventually lead to the incorrect deformation path (see Figure 16a). The $\{\boldsymbol{p}, \boldsymbol{F}\}$ JST-SPH methodology is not able to resolve the above issues for large values of the Poisson's ratio (typically, for $\nu>0.495$ ) (see Figure 16b). In this case, a new additional conservation law for the Jacobian $J$ is necessary in order to 
$t=0.00022 \mathrm{~s}$

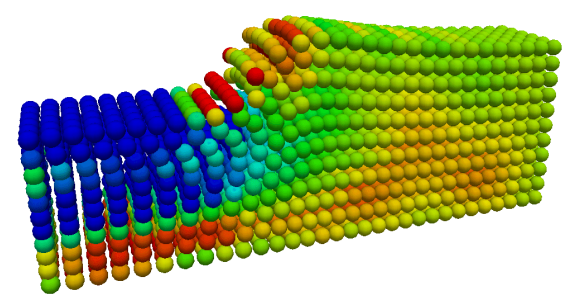

$t=0.00054 \mathrm{~s}$

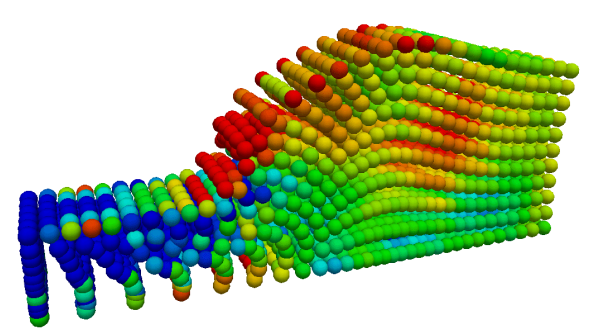

(a) Mixed-based $\{\boldsymbol{p}, \boldsymbol{F}\} \operatorname{SPH}\left(\varepsilon_{\boldsymbol{p}}^{(2)}=0\right.$ and $\left.\varepsilon_{\boldsymbol{p}}^{(4)}=0\right)$
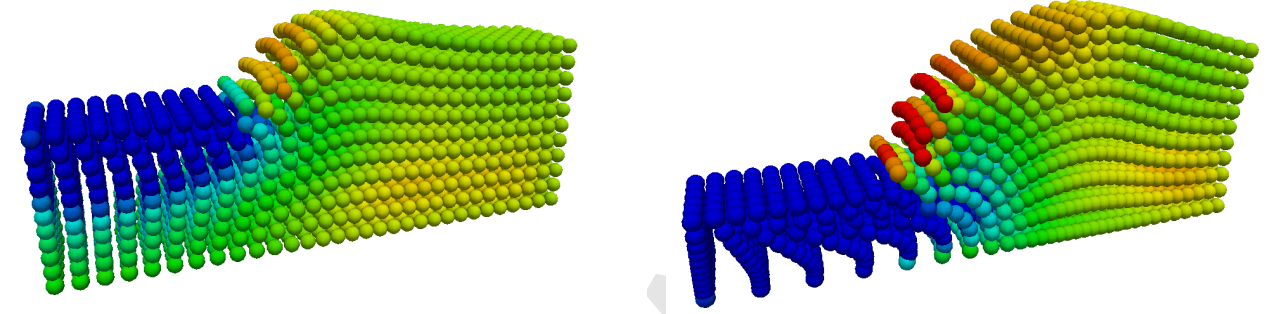

(b) Mixed-based $\{\boldsymbol{p}, \boldsymbol{F}\}$ JST-SPH $\left(\varepsilon_{\boldsymbol{p}}^{(2)}=0\right.$ and $\left.\varepsilon_{\boldsymbol{p}}^{(4)}=\frac{1}{8}\right)$
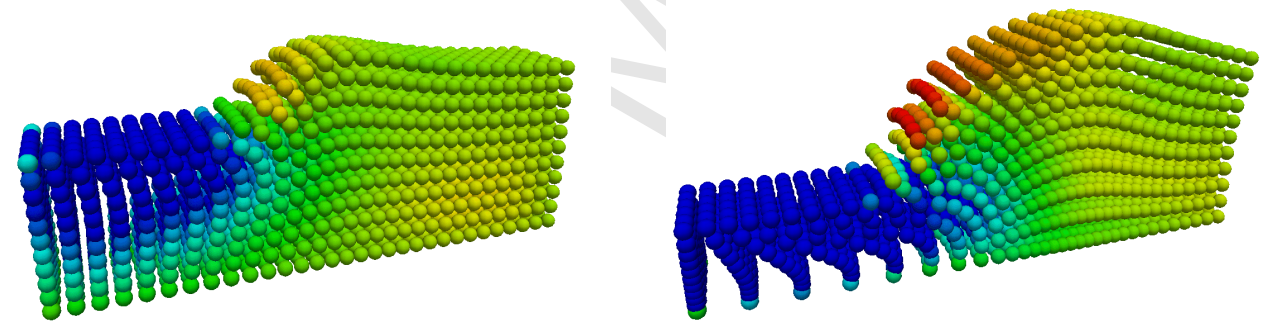

(c) Mixed-based $\{\boldsymbol{p}, \boldsymbol{F}, J\}$ JST-SPH $\left(\varepsilon_{\boldsymbol{p}}^{(2)}=\varepsilon_{J}^{(2)}=\varepsilon_{J}^{(4)}=0\right.$ and $\left.\varepsilon_{\boldsymbol{p}}^{(4)}=\frac{1}{8}\right)$
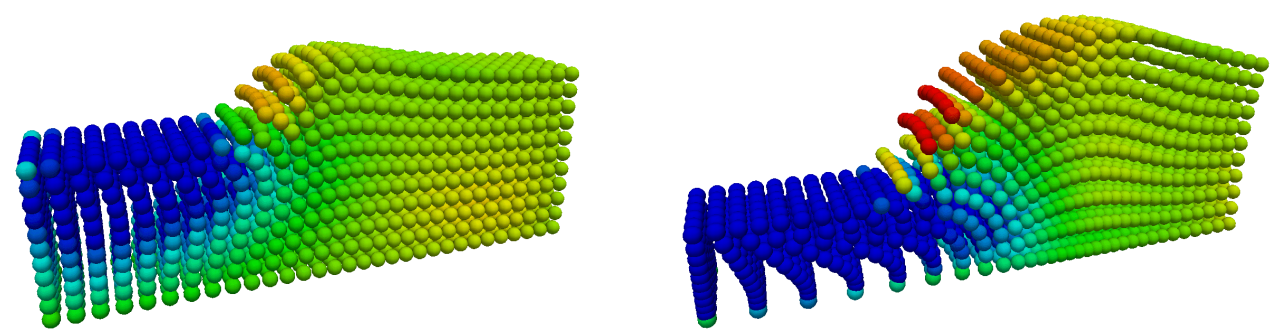

(d) Mixed-based $\{\boldsymbol{p}, \boldsymbol{F}, \boldsymbol{H}, J\}$ JST-SPH $\left(\varepsilon_{\boldsymbol{p}}^{(2)}=\varepsilon_{J}^{(2)}=\varepsilon_{J}^{(4)}=0\right.$ and $\left.\varepsilon_{\boldsymbol{p}}^{(4)}=\frac{1}{8}\right)$ $-8.000+05$ $-3 e+5$

$4.000 e+05$

\section{Pressure $(\mathrm{Pa})$}

Figure 13: Symmetric coining: Timg7evolution of the deformation plotted with pressure distribution using (a) $\{\boldsymbol{p}, \boldsymbol{F}\}$ SPH; (b) $\{\boldsymbol{p}, \boldsymbol{F}\}$ JST-SPH; (c) $\{\boldsymbol{p}, \boldsymbol{F}, J\}$ JST-SPH; and (d) $\{\boldsymbol{p}, \boldsymbol{F}, \boldsymbol{H}, J\}$ JST-SPH. A neo-Hookean material is used with density $\rho_{0}=1000 \mathrm{~kg} / \mathrm{m}^{3}$, Young's modulus $E=1 \mathrm{MPa}$, Poisson's ratio $\nu=0.495$ and $\alpha_{C F L}=0.3$. Discretisation of $31 \times 11 \times 6$ particles. 
$t=0 \mathrm{~s}$

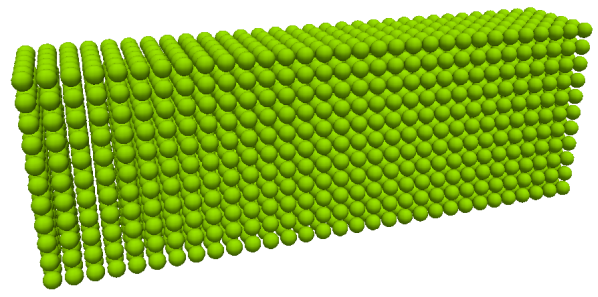

$t=0.00025 \mathrm{~s}$

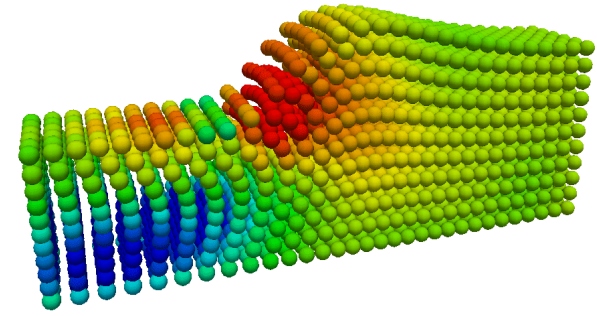

$t=0.0004 \mathrm{~s}$

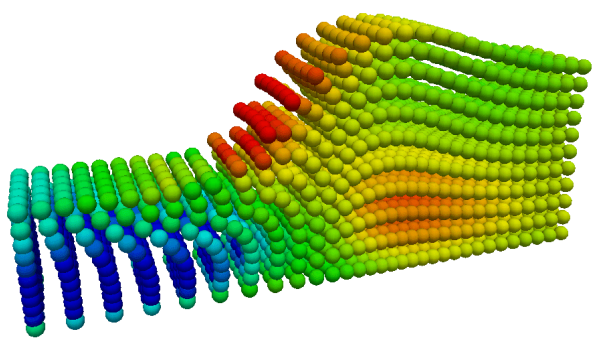

$t=0.00052 \mathrm{~s}$

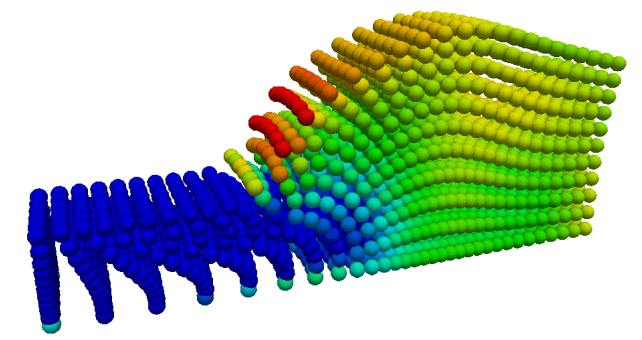

$t=0.00015 \mathrm{~s}$

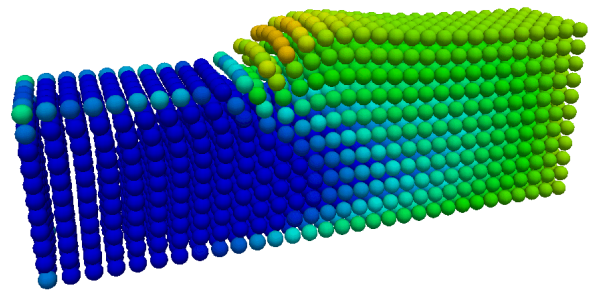

$t=0.00032 \mathrm{~s}$

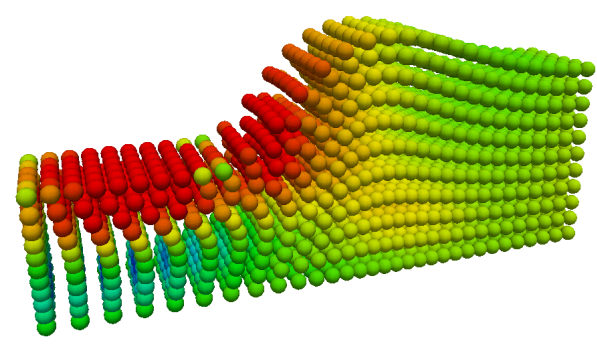

$t=0.00046 \mathrm{~s}$

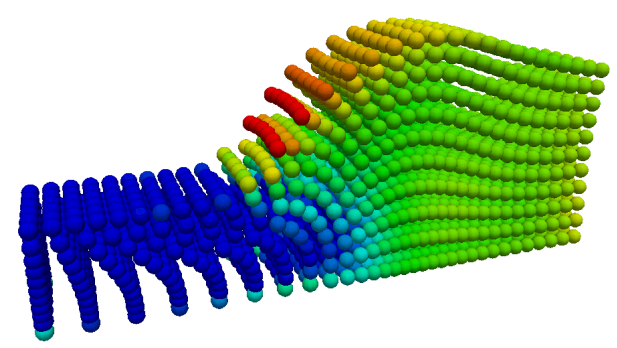

$t=0.00058 \mathrm{~s}$

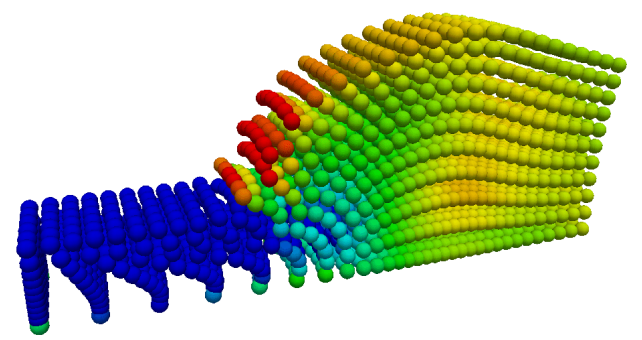

$-3 e+5$

$4.000 e+05$

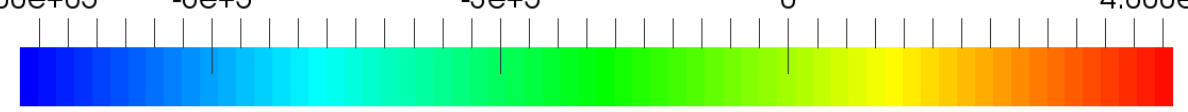

Pressure $(\mathrm{Pa})$

Figure 14: Symmetric coining: Time evolution of the deformation plotted with pressure distribution using $\left\{\boldsymbol{p}, \boldsymbol{F}^{3}\right\}$ JST-SPH. A neo-Hookean material is used with density $\rho_{0}=1000 \mathrm{~kg} / \mathrm{m}^{3}$, Young's modulus $E=1 \mathrm{MPa}$, Poisson's ratio $\nu=0.495$ and $\alpha_{C F L}=0.3$. JST parameters used: $\varepsilon_{\boldsymbol{p}}^{(2)}=0$ and $\varepsilon_{\boldsymbol{p}}^{(4)}=\frac{1}{8}$. Discretisation of $31 \times 11 \times 6$ particles. 


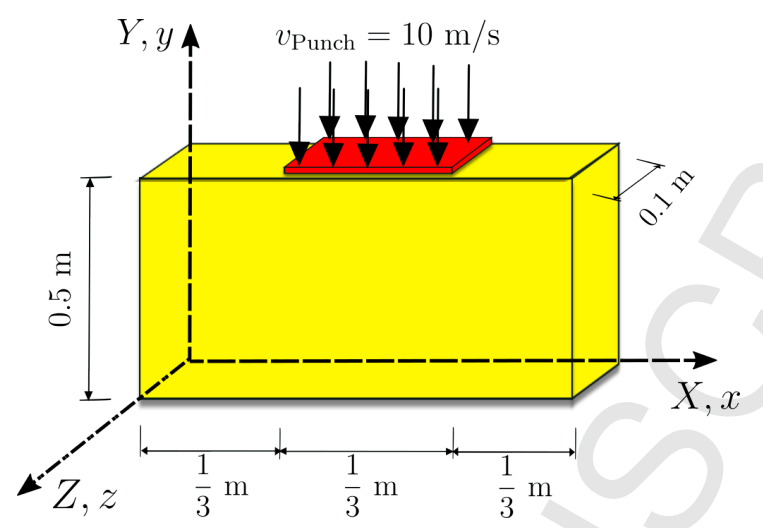

Figure 15: Punch test configuration

stabilise the overall formulation, as already reported by the authors in previous publications [42]. A smooth pressure spatial representation (see Figures 16c, 16d) can be observed when using $\{\boldsymbol{p}-\boldsymbol{F}-\} J$ and $\{\boldsymbol{p}-\boldsymbol{F}-\boldsymbol{H}-J\}$ JST-SPH methodologies.

\subsection{Alternative version of punch example: Inertial effects}

Similarly to the examples discussed above (see Section (8.5) and Section (8.6)), a unit block of $1 \mathrm{~m} \times 1 \mathrm{~m} \times 1 \mathrm{~m}$ (which is free on its top side and is constrained with rollers on the rest of the boundaries) is here presented [37] (see Figure 17). In previous cases, the inertial effect contributions can be considered to be relatively small due to the constant application of the boundary velocity. On the contrary, large inertial effects will be explored in this example. With this in mind, a velocity field $\boldsymbol{v}_{0}=(0,-100,0)^{T} \mathrm{~m} / \mathrm{s}$ is first applied on the mid quarter of the top side to be then released after time $t=0.007 \mathrm{~s}$. A nearly incompressible neo-Hookean model is used with material density $\rho_{0}=1100 \mathrm{~kg} / \mathrm{m}^{3}$, Young's modulus $E=17 \mathrm{MPa}$ and Poisson's ratio $\nu=0.45\left(\frac{\kappa}{\mu} \approx 10\right)$. From Figures (18a) and (18b), excessive pressure fluctuations can be detected using either the classical displacementbased SPH or the unstabilised $\{\boldsymbol{p}, \boldsymbol{F}\}$ SPH. The pressure-related spurious mechanism can be entirely eliminated with the inclusion of appropriate JST stabilisation terms $\left(\varepsilon_{\boldsymbol{p}}^{(4)}=\frac{1}{8}\right)$ when using the $\{\boldsymbol{p}, \boldsymbol{F}\}$ JST-SPH methodology (see Figure 18c).

We can now further examine the problem by using a more sophisticated Mooney-Rivlin model (8) dominated by the $\boldsymbol{H}$-term (by setting $\varsigma=0$ and 

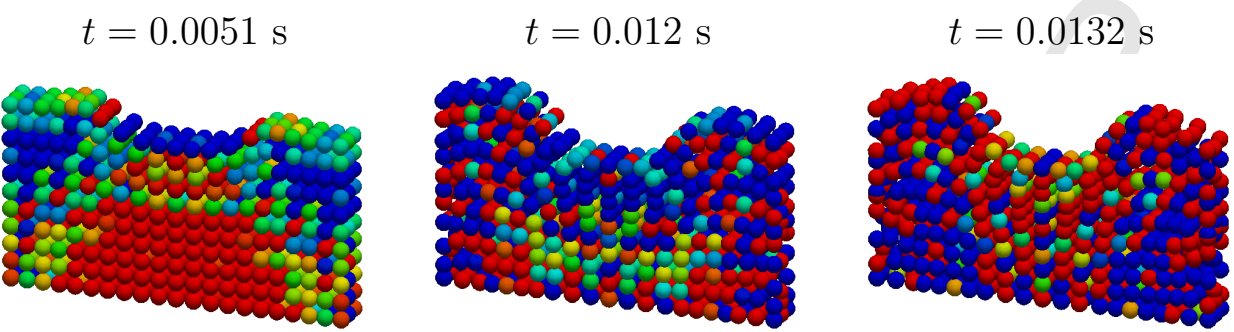

(a) Displacement-based SPH
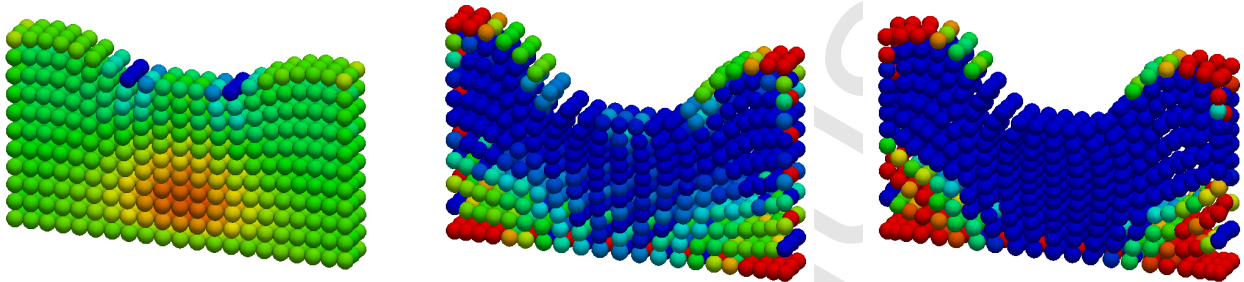

(b) Mixed-based $\{\boldsymbol{p}, \boldsymbol{F}\}$ JST-SPH $\left(\varepsilon_{\boldsymbol{p}}^{(2)}=0\right.$ and $\left.\varepsilon_{\boldsymbol{p}}^{(4)}=\frac{1}{8}\right)$
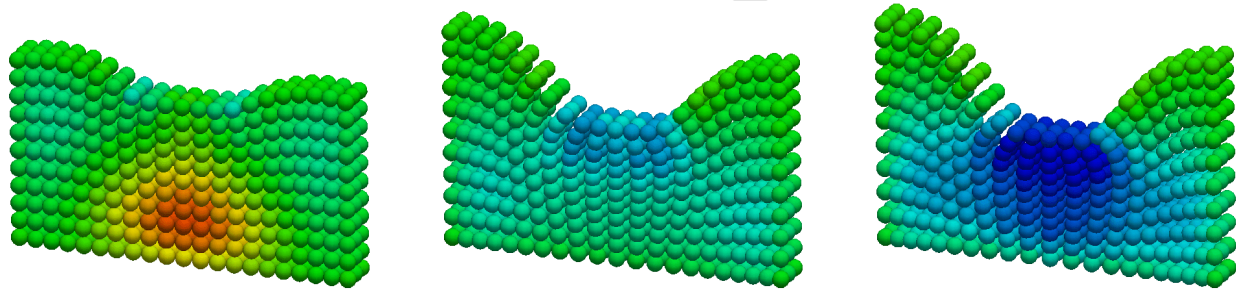

(c) Mixed-based $\{\boldsymbol{p}, \boldsymbol{F}, J\}$ JST-SPH $\left(\varepsilon_{\boldsymbol{p}}^{(2)}=\varepsilon_{J}^{(2)}=0, \varepsilon_{\boldsymbol{p}}^{(4)}=\frac{1}{8}\right.$ and $\left.\varepsilon_{J}^{(4)}=\frac{1}{32}\right)$
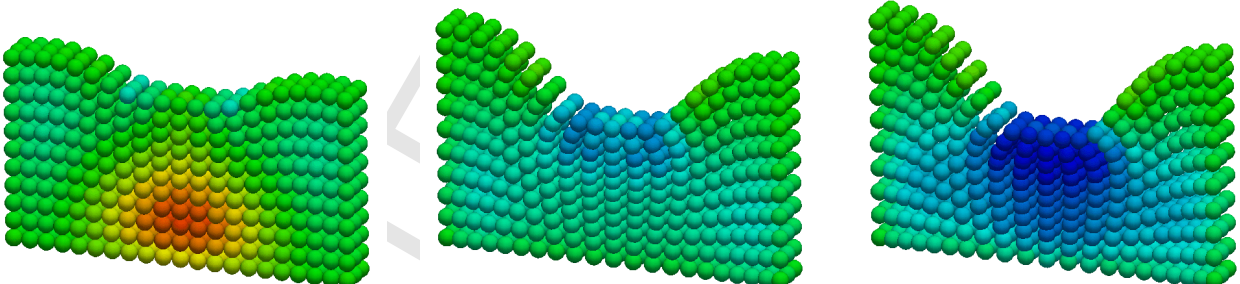

(d) Mixed-based $\{\boldsymbol{p}, \boldsymbol{F}, \boldsymbol{H}, J\}$ JST-SPH $\left(\varepsilon_{\boldsymbol{p}}^{(2)}=\varepsilon_{J}^{(2)}=0, \varepsilon_{\boldsymbol{p}}^{(4)}=\frac{1}{8}\right.$ and

$$
\left.\varepsilon_{J}^{(4)}=\frac{1}{32}\right)
$$

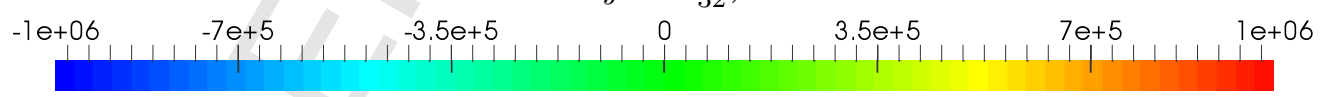

\section{Pressure $(\mathrm{Pa})$}

Figure 16: Nearly incompressible punch test: Time evolution of the deformation plotted with pressure distribution using (a) Displacement-based SPH; (b) $\{\boldsymbol{p}, \boldsymbol{F}\}$ JST-SPH; (c) $\boldsymbol{p}$ - $\boldsymbol{F}$-J JST-SPH; and (d) $\boldsymbol{p}$ - $\boldsymbol{F}$ - $\boldsymbol{H}$-J JST-SPH. A neo-Hookean material is used with density $\rho_{0}=1000 \mathrm{~kg} / \mathrm{m}^{3}$, Young's modulus $E=1 \mathrm{MPa}$, Poisson's ratio $\nu=0.499$ and $\alpha_{C F L}=0.3$. Dicretisation of $21 \times 11 \times 3$ particles. 


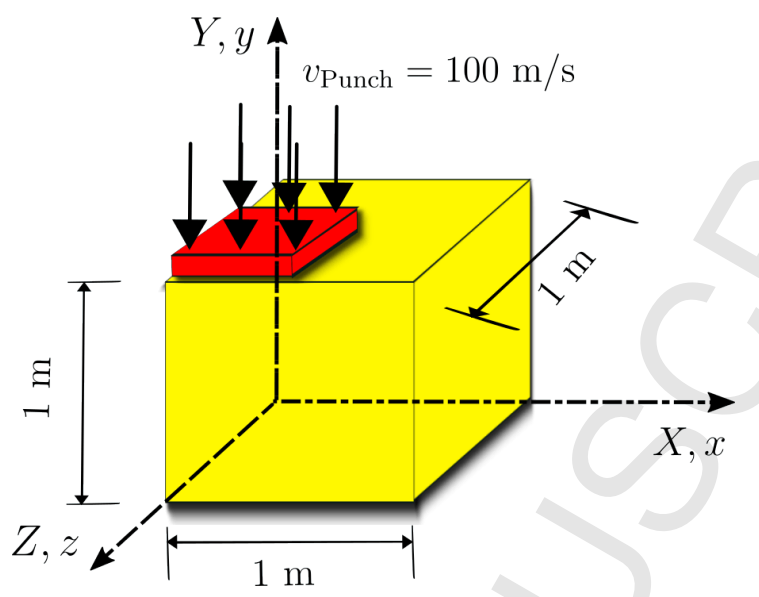

Figure 17: Punch cube configuration

$\left.\xi=\frac{\mu}{3 \sqrt{3}}\right)$. It is interesting to see that both $\{\boldsymbol{p}, \boldsymbol{F}, J\}$ and $\{\boldsymbol{p}, \boldsymbol{F}, \boldsymbol{H}, J\}$ JST-SPH methods produce practically identical results that are freed from non-physical mechanisms similar to hourglassing (see Figure 19).

For the sake of completeness, time evolution of the deformed block is also shown in Figure 20. Clearly, the mixed-based $\{\boldsymbol{p}, \boldsymbol{F}\}$ JST-SPH method can be employed without any difficulties dealing with extremely large deformations.

\subsection{Bending column}

Following References $[1,2,17,18,37,41-44,71]$, this example shows a 1 $\mathrm{m}$ squared cross section column clamped at the bottom and free on all other sides. An initial linear variation of the velocity profile is prescribed in the $X-Y$ plane given by $\boldsymbol{v}_{0}=V[Y / L, 0,0]^{T}$, where $V=10 \mathrm{~m} / \mathrm{s}$ and $L=6 \mathrm{~m}$ is the length of the column (see Figure 21).

As reported in References $[17,18]$ (see Section 2.4.1 on pg. 2693-2695 in [17] and Section 4 on pg. 1218 in [18]), this bending problem was successfully solved using the Total Lagrangian CSPH framework without the need to use artificial viscosity. However, results are only displayed for a relatively short period of time.

The main objective of this problem is to demonstrate the performance of the proposed JST-SPH methodologies in nearly incompressible bending dominated scenarios over a long-term response. A nearly incompressible neo-Hookean model is used where the material properties are density $\rho_{0}=$ 


$$
t=0.0081 \mathrm{~s}
$$
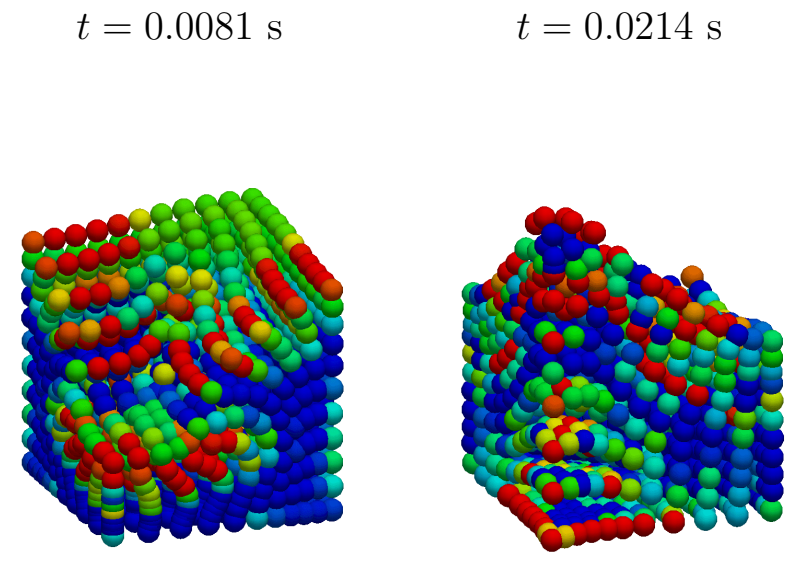

(a) Displacement-based SPH
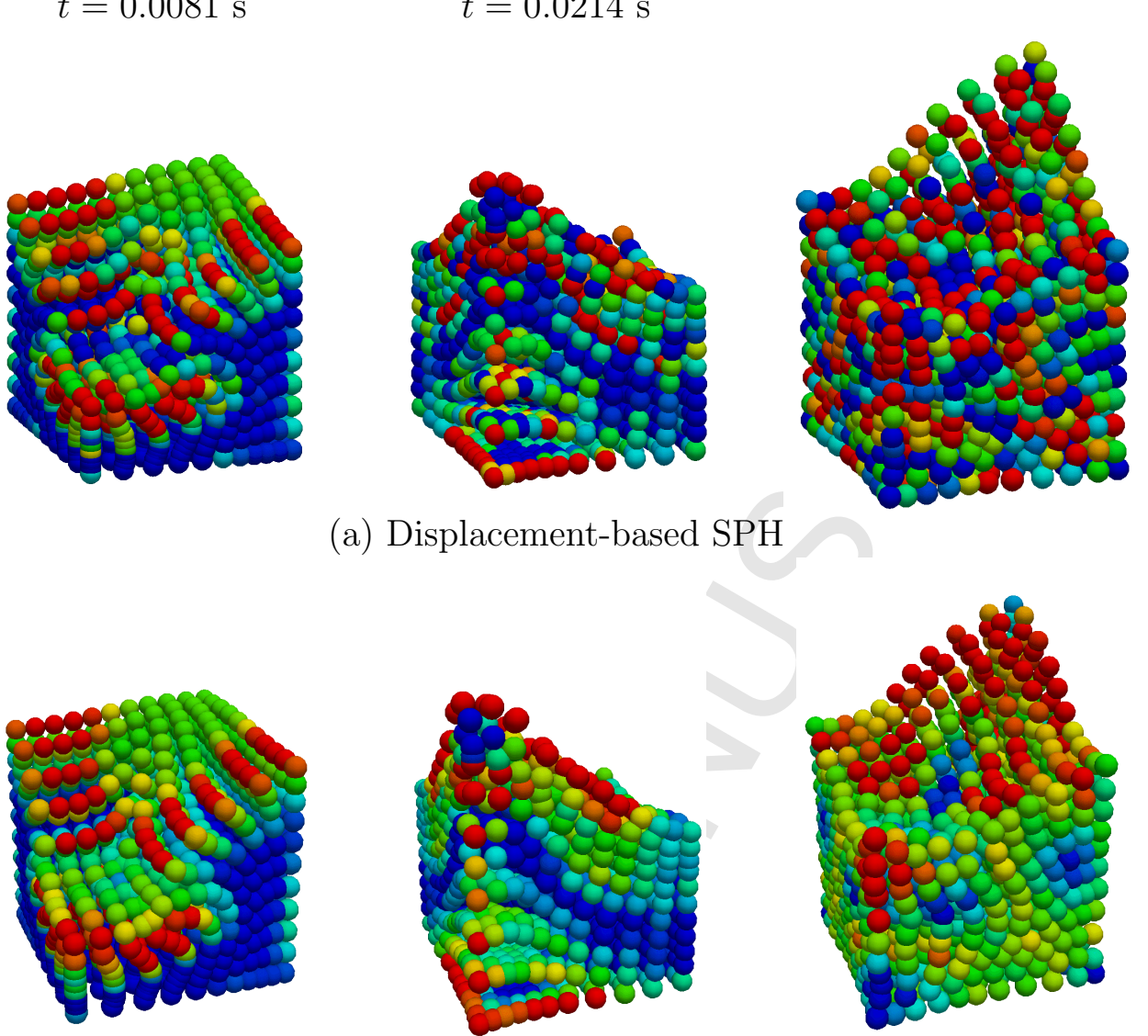

(b) Mixed-based $\{\boldsymbol{p}, \boldsymbol{F}\} \operatorname{SPH}\left(\varepsilon_{\boldsymbol{p}}^{(2)}=0\right.$ and $\left.\varepsilon_{\boldsymbol{p}}^{(4)}=0\right)$
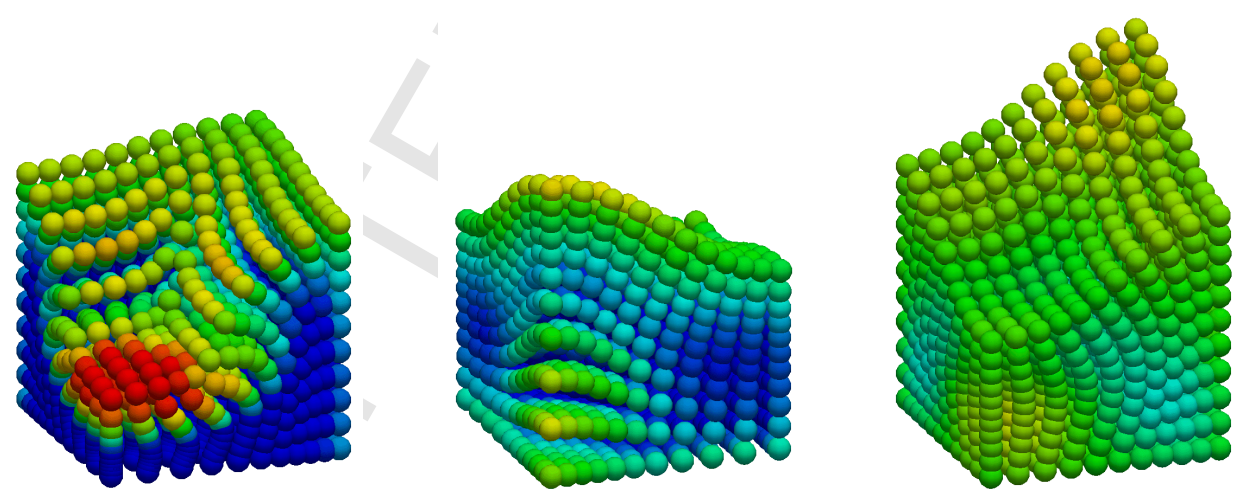

(c) Mixed-based $\{\boldsymbol{p}, \boldsymbol{F}\}$ JST-SPH $\left(\varepsilon_{\boldsymbol{p}}^{(2)}=0\right.$ and $\left.\varepsilon_{\boldsymbol{p}}^{(4)}=\frac{1}{8}\right)$

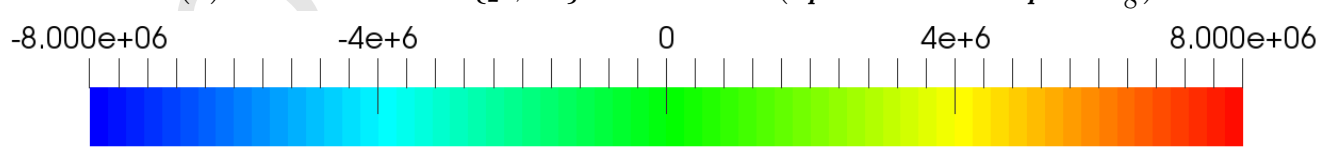

\section{Pressure $(\mathrm{Pa})$}

Figure 18: Nearly incompressible punch test: Time evolution of the deformation plotted with pressure distributiłß using (a) Displacement-based SPH; (b) Mixed-based $\{\boldsymbol{p}, \boldsymbol{F}\}$ SPH; and (c) Mixed-based $\{\boldsymbol{p}, \boldsymbol{F}\}$ JST-SPH. A neoHookean material is used with density $\rho_{0}=1100 \mathrm{~kg} / \mathrm{m}^{3}$, Young's modulus $E=17 \mathrm{MPa}$, Poisson's ratio $\nu=0.45$ and $\alpha_{C F L}=0.3$. Dicretisation of $11 \times 11 \times 11$ particles. 
$t=0.0083 \mathrm{~s}$

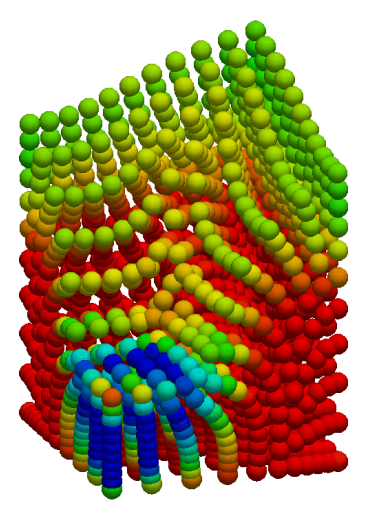

$t=0.0191 \mathrm{~s}$

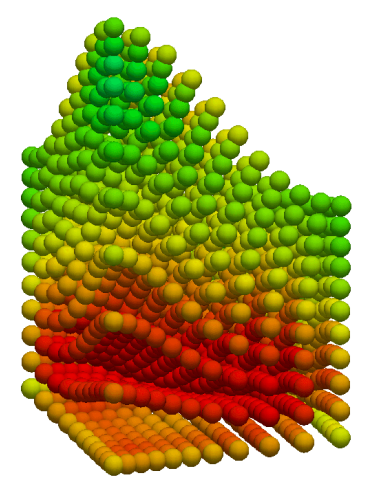

$t=0.0313 \mathrm{~s}$

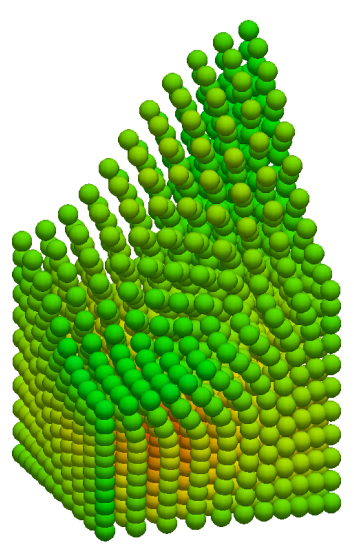

(a) Mixed-based $\{\boldsymbol{p}, \boldsymbol{F}, J\}$ JST-SPH
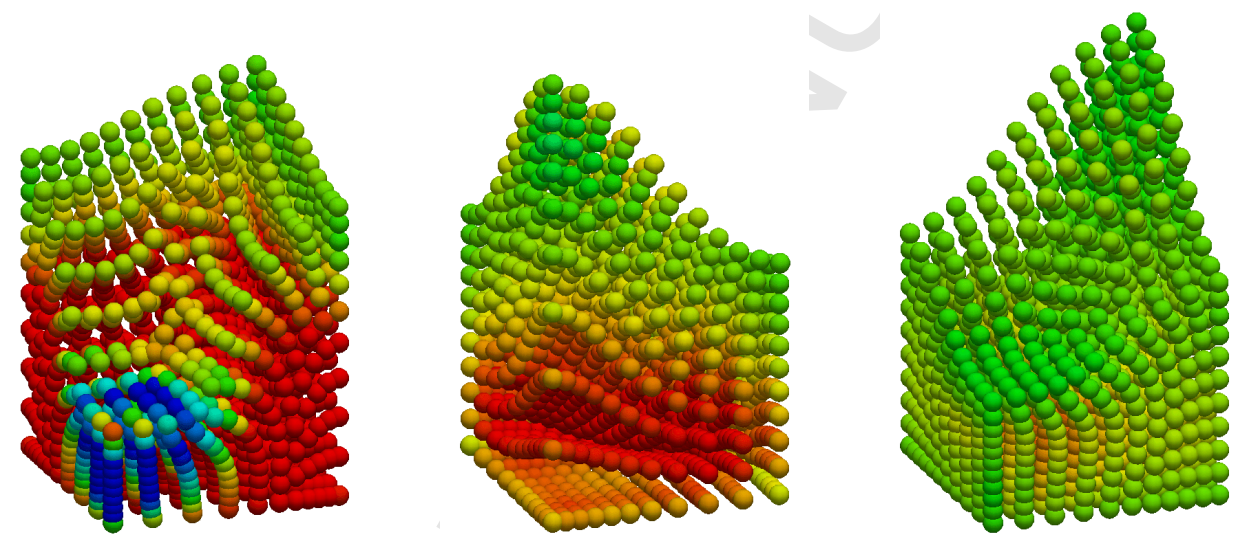

(b) Mixed-based $\{\boldsymbol{p}, \boldsymbol{F}, \boldsymbol{H}, J\}$ JST-SPH

$-3.000 e+07$ $-1.5 e+7$ 0

\section{Pressure $(\mathrm{Pa})$}

Figure 19: Nearly incompressible punch test: Time evolution of the deformation plotted with pressure distribution using (a) Mixed-based $\{\boldsymbol{p}, \boldsymbol{F}, J\}$ JST-SPH; and (b) Mixed-based $\{\boldsymbol{p}, \boldsymbol{F}, \boldsymbol{H}, J\}$ JST-SPH. A Mooney-Rivlin material $\left(\varsigma=0, \xi=\frac{\mu}{3 \sqrt{3}}\right)$ is used with density $\rho_{0}=1100 \mathrm{~kg} / \mathrm{m}^{3}$, Young's modulus $E=17 \mathrm{MPa}$, Poisson's ratio $\nu=0.495$ and $\alpha_{C F L}=0.3$. Dicretisation of $11 \times 11 \times 11$ particles. JST parameters used: $\varepsilon_{\boldsymbol{p}}^{(2)}=\varepsilon_{J}^{(2)}=0, \varepsilon_{\boldsymbol{p}}^{(4)}=\frac{1}{8}$ and $\varepsilon_{J}^{(4)}=\frac{1}{32}$. 


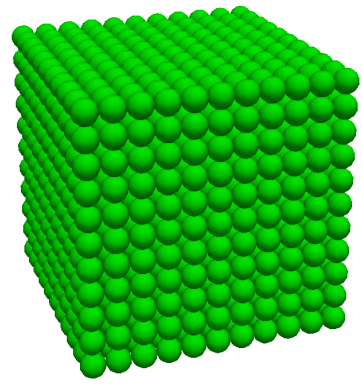

$t=0 \mathrm{~s}$

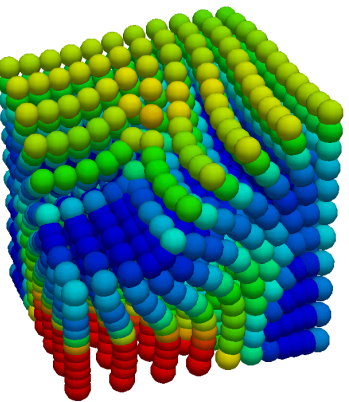

$t=0.0089 \mathrm{~s}$

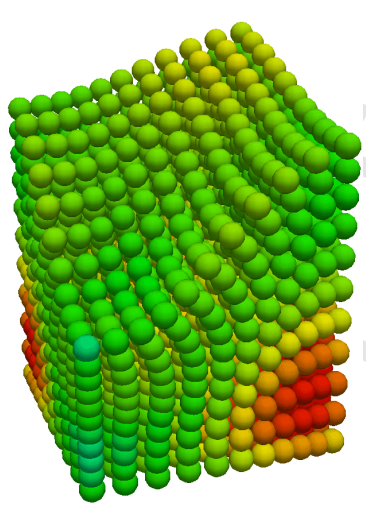

$t=0.0373 \mathrm{~s}$

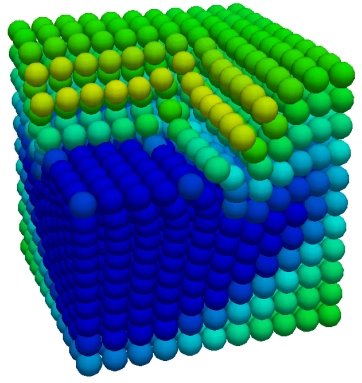

$t=0.0035 \mathrm{~s}$

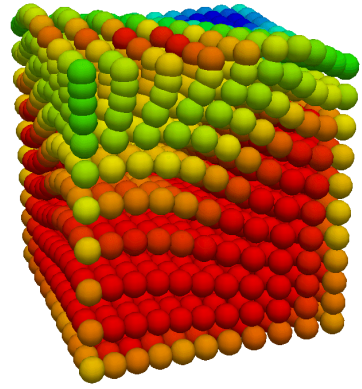

$t=0.0173 \mathrm{~s}$

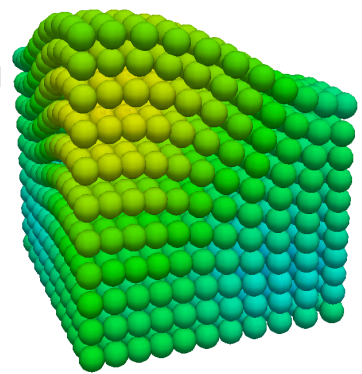

$t=0.047 \mathrm{~s}$

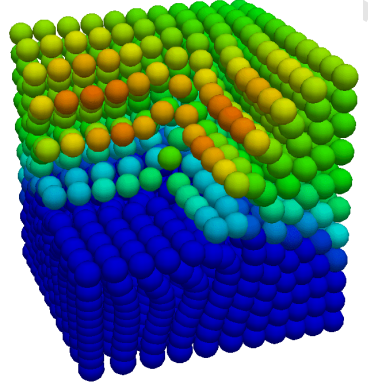

$t=0.006 \mathrm{~s}$

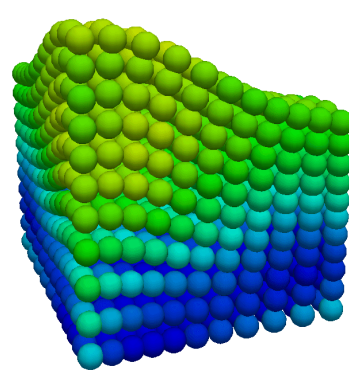

$t=0.028 \mathrm{~s}$

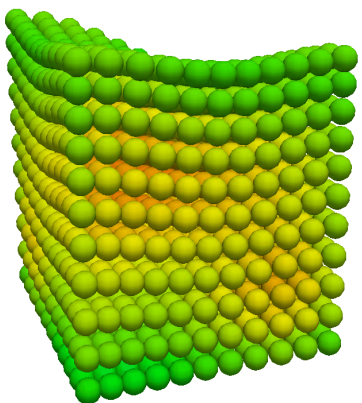

$t=0.0577 \mathrm{~s}$

$8.000 e+06$

Figure 20: Alternative punch test: Time evolution of the deformation plotted with pressure distribution using the mixed-based $\{\boldsymbol{p}, \boldsymbol{F}\}$ JST-SPH. A neoHookean material is used with density $\rho_{0}=1100 \mathrm{~kg} / \mathrm{m}^{3}$, Young's modulus $E=17 \mathrm{MPa}$, Poisson's ratio $\nu=0.45$ and $\alpha_{C F L}=0.3$. JST parameters used: $\varepsilon_{\boldsymbol{p}}^{(2)}=0$ and $\varepsilon_{\boldsymbol{p}}^{(4)}=\frac{1}{8}$. Dicretisation of $11 \times 11 \times 11$ particles. 


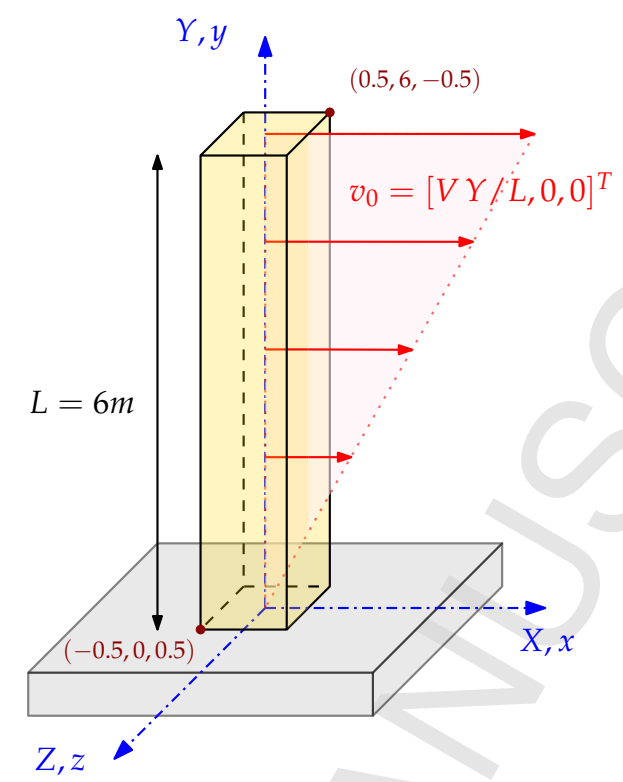

Figure 21: Bending column configuration

$1100 \mathrm{~kg} / \mathrm{m}^{3}$, Young's modulus $E=17 \mathrm{MPa}$ and Poisson's ratio $\nu=0.45$ $\left(\frac{\kappa}{\mu} \approx 10\right)$. For comparison purposes, an ample spectrum of alternative numerical strategies will be employed, namely Constrained-TOUCH [36], Hyperelastic-GLACE [47], Upwind-VCFVM [44], JST-VCFVM [43], PGFEM [1, 2, 41, 42], non-LBB compliant B-bar (or known as P1-Q0) hexahedral element [8] and the LBB compliant $\mathrm{Hu}$-Washizu tetrahedral element [49]. Figure 22 compares the deformed shape and the pressure contour for the above-mentioned eight different methodologies. As can be observed, the results of the scheme proposed in this paper (with a discretisation of $5 \times 25 \times 5$ particles) match very well those of the other numerical strategies.

We now turn the attention to assess the performance of the proposed algorithm in the near incompressibility regime with $\nu=0.499\left(\frac{\kappa}{\mu} \approx 500\right)$. As discussed in Section 8.6, an extra conservation law for the Jacobian $J$ is necessary. Figure 23 shows the importance of the introduction of $J$ when considering highly nonlinear nearly incompressible scenarios. The $\{\boldsymbol{p}, \boldsymbol{F}\}$ JST-SPH methodology clearly exhibits an unrealistic pressure pattern, as shown in Figure 23a. When resorting to the mixed-based $\{\boldsymbol{p}, \boldsymbol{F}, J\}$ JST$\mathrm{SPH}$ methodology, it is remarkable the smooth pressure contour observed with a discretisation of only three particles across the thickness (see Figure 
$23 b)$.

\subsection{Twisting column}

In order to examine the robustness of the scheme, a more challenging example proposed in References $[1,2,42-44,71]$ is considered in this section. The problem is initialised with a sinusoidal angular velocity field relative to the origin given by $\boldsymbol{\omega}_{0}=[0, \Omega \sin (\pi Y / 2 L), 0]^{T}$ where $\Omega=105 \mathrm{rad} / \mathrm{s}$ and $L=$ $6 \mathrm{~m}$ is the length of the column (see Figure 24). A nearly incompressible neoHookean material is used and the following material properties are density $\rho_{0}=1100 \mathrm{~kg} / \mathrm{m}^{3}$, Young's modulus $E=17 \mathrm{MPa}$ and Poisson's ratio $\nu=0.45$ $\left(\frac{\kappa}{\mu} \approx 10\right)$.

A sequence of successively refined meshes $(4 \times 19 \times 4,5 \times 25 \times 5,6 \times 31 \times 6$ and $7 \times 37 \times 7)$ using the mixed-based $\{\boldsymbol{p}, \boldsymbol{F}\}$ JST-SPH framework is shown in Figure 25. Overly flexible behaviour (see Figure 25a) is clearly seen when using very few particles in the simulation. A more realistic deformation path, as well as pressure representation, can be obtained by increasing the number of particles (see Figure 25d). It is also interesting to notice how the methodology preserves perfect axial rotation introducing no out-of-axis characteristics.

For benchmarking purposes, we solve the exact same problem using other available numerical methodologies, namely, Upwind-VCFVM [44], JST-VCFVM [43], the LBB compliant Hu-Washizu type variational principle [49], ConstrainedTOUCH [36], Hyperelastic-GLACE [47] and the non-LBB compliant B-bar (or known as P1-Q0) hexahedral element [8]. As shown in Figure 26, both deformations and pressure patterns predicted by the mixed-based $\{\boldsymbol{p}, \boldsymbol{F}\}$ JST-SPH framework agree very well with other published methodologies. The pressure contour displayed for the B-bar element is shown piecewise constant per element and not nodally interpolated ${ }^{6}$.

Figure 27 includes a similar comparative study, with a reduced number of methodologies and with a higher Poisson's ratio $\nu=0.495$, which can be considered as very nearly incompressible $\left(\frac{\kappa}{\mu} \approx 100\right)$. In this case, the simulation could not be solved using the JST-VCFVM, the UpwindVCFVM and Hyperelastic-GLACE schemes. Crucially, all computational

\footnotetext{
${ }^{6}$ Nodal averaging is sometimes carried out in order to smooth possible pressure oscillations [8].
} 


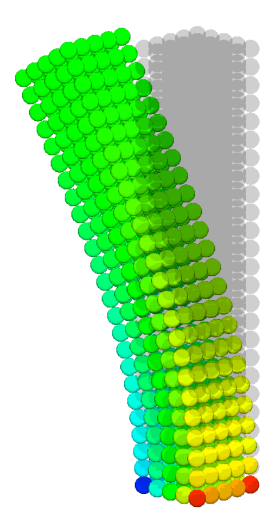

(a)

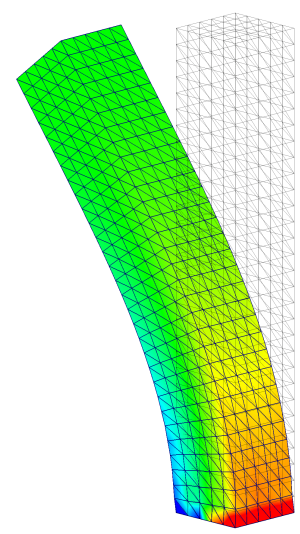

(e)

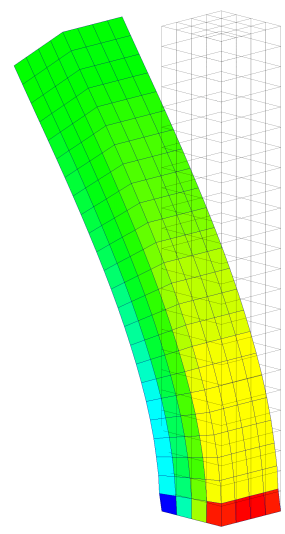

(b)

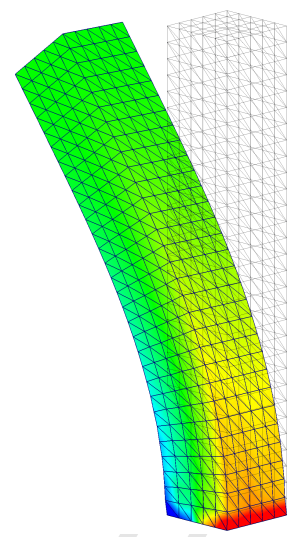

(f)

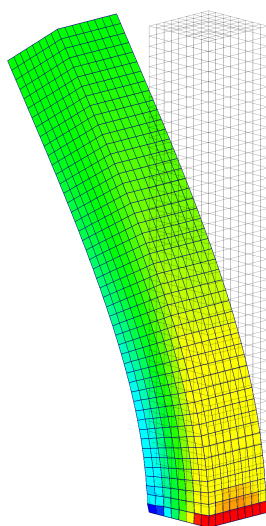

(c)

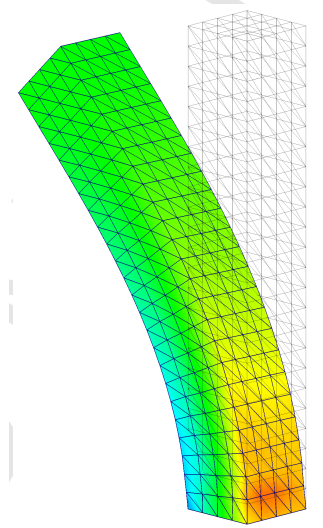

(g)

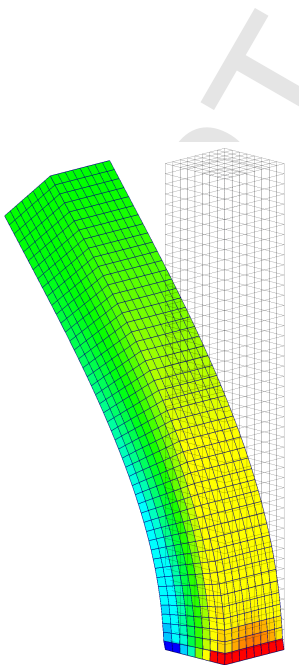

(d)

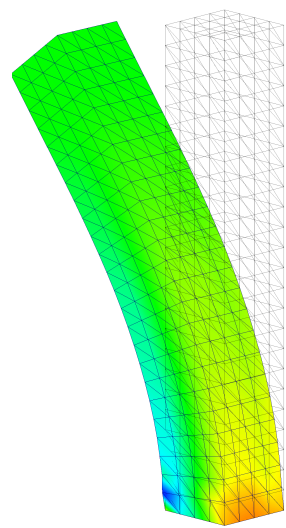

(h)

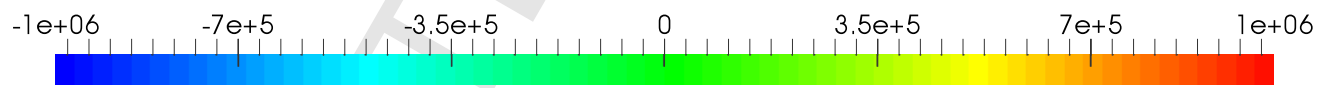

\section{Pressure $(\mathrm{Pa})$}

Figure 22: Bending column: Comparison of deformed shapes plotted with pressures at time $t=1.5 \mathrm{~s}$ using various numerical schemes: (a) Mixed-based $\{\boldsymbol{p}, \boldsymbol{F}\}$ JST-SPH $\left(\varepsilon_{\boldsymbol{p}}^{(2)}=0\right.$ and $\left.\varepsilon_{\boldsymbol{p}}^{(4)}=\frac{1}{8}\right)$; (b) B-bar hexahedral method [8]; (c) Hyperelastic-GLACE [47]; (d) Constrained-TOUCH [36]; (e) UpwindVCFVM [44]; (f) JST-VCFVM [43]; (g) PG-FEM [42]; and (h) Hu-Washizu type variational principle [49]. Results are obtained with a linear variation of velocity field $\boldsymbol{v}_{0}=[V Y / L, 0,0]^{T}$ where $V=10 \mathrm{~m} / \mathrm{s}$ and $L=6 \mathrm{~m}$. A neoHookean material is used with density $\rho_{0}=1100 \mathrm{~kg} / \mathrm{m}^{3}$, Young's modulus $E=17 \mathrm{MPa}$ and Poisson's ratio $\nu=0.45$. 
$t=0.35 \mathrm{~s}$

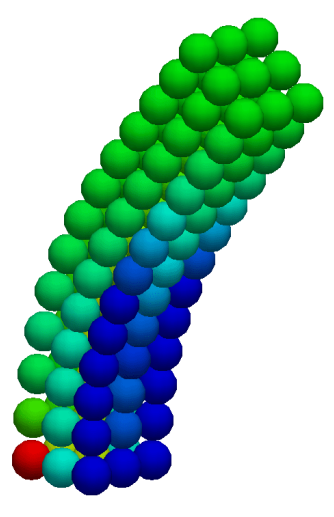

$t=0.69 \mathrm{~s}$

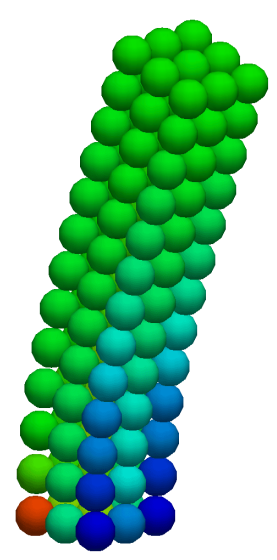

$t=1.03 \mathrm{~s}$

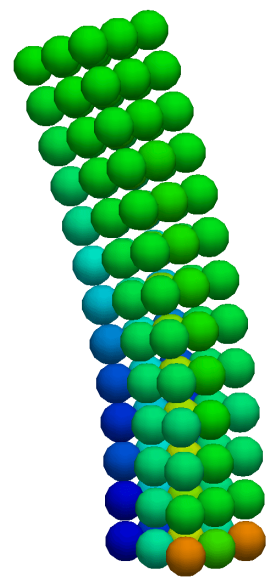

$t=1.37 \mathrm{~s}$

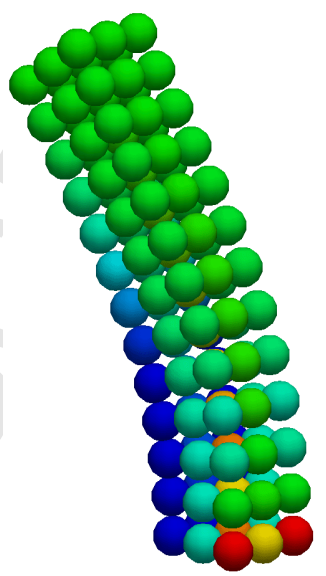

(a) Mixed-based $\{\boldsymbol{p}, \boldsymbol{F}\} \operatorname{JST}-\mathrm{SPH}\left(\varepsilon_{\boldsymbol{p}}^{(2)}=0\right.$ and $\left.\varepsilon_{\boldsymbol{p}}^{(4)}=\frac{1}{8}\right)$
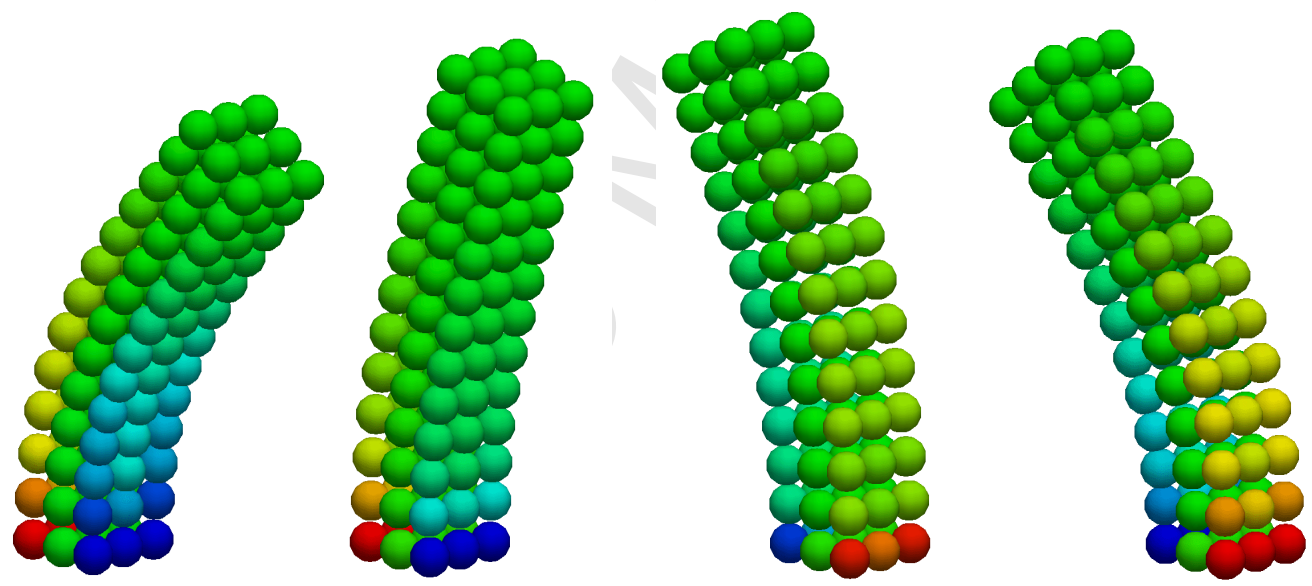

(b) Mixed-based $\{\boldsymbol{p}, \boldsymbol{F}, J\}$ JST-SPH $\left(\varepsilon_{\boldsymbol{p}}^{(2)}=\varepsilon_{J}^{(2)}=0, \varepsilon_{\boldsymbol{p}}^{(4)}=\frac{1}{8}\right.$ and $\left.\varepsilon_{J}^{(4)}=\frac{1}{32}\right)$ $-1 e+06$ $-7 e+5$ $-3.5 e+5$ $0 \quad 3.5 e+5$ $7 e+5$

\section{Pressure $(\mathrm{Pa})$}

Figure 23: Bending column: Comparison of deformed shapes plotted with pressures using (a) Mixed-based $\{\boldsymbol{p}, \boldsymbol{F}\}$ JST-SPH; and (b) Mixed-based $\{\boldsymbol{p}, \boldsymbol{F}, J\}$ JST-SPH. Results are obtained with a linear variation of velocity field $\boldsymbol{v}_{0}=[V Y / L, 0,0]^{T}$ where $V=10 \mathrm{~m} / \mathrm{s}$ and $L=6 \mathrm{~m}$. A neo-Hookean material is used with density $\rho_{0}=1100 \mathrm{~kg} / \mathrm{m}^{3}$, Young's modulus $E=17$ $\mathrm{MPa}$, Poisson's ratio $\nu=0.499$ and $\alpha_{48}{ }^{F L}=0.3$. Discretisation of $3 \times 18 \times 3$ particles. 


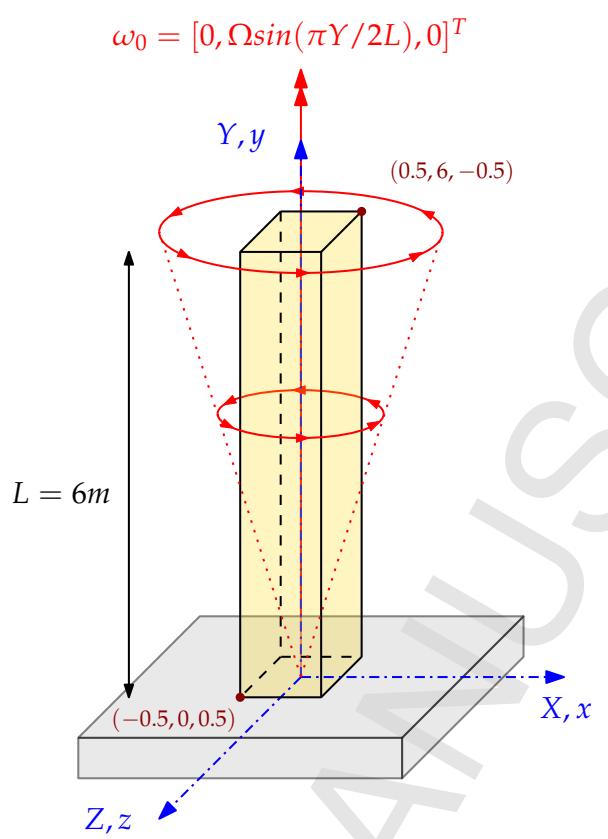

Figure 24: Twisting column configuration

mixed-based methodologies displayed produce very similar deformation patterns with smooth pressure distribution.

For extremely high values of the Poisson's ratio $\nu=0.499\left(\frac{\kappa}{\mu} \approx 500\right)$, the mixed-based $\{\boldsymbol{p}, \boldsymbol{F}\}$ JST-SPH framework, as expected, shows non-physical pressure fluctuations throughout the domain. This will eventually lead to the breakdown of a numerical scheme (see Figure 28a). On the contrary, the mixed-based $\{\boldsymbol{p}, \boldsymbol{F}, J\}$ JST-SPH algorithm produces reliable results that are freed from zero energy modes (see Figure 28b).

\section{Conclusions}

This paper introduces a mixed stabilised Total Lagrangian Smooth Particle Hydrodynamics (SPH) computational framework for the numerical analysis of large strain fast solid dynamics. Following References [1, 2], a mixedbased $\{\boldsymbol{p}, \boldsymbol{F}, \boldsymbol{H}, J\}$ set of equations is written in the form of a system of first order conservation laws. The linear momentum $\boldsymbol{p}$ and the set of geometric strain measures, namely the deformation gradient tensor $\boldsymbol{F}$, the co-factor $\boldsymbol{H}$ and the Jacobian $J$, are regarded as primary conservation variables. 
$t=0.1 \mathrm{~s}$

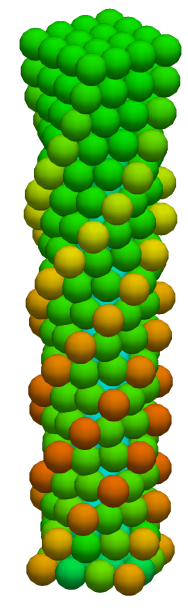

(a) $4 \times 4 \times 19$

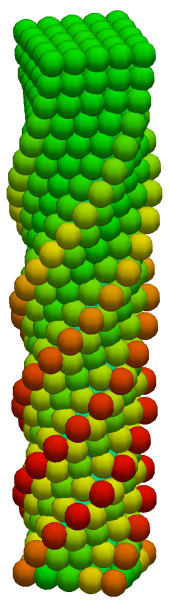

(b) $5 \times 5 \times 25$

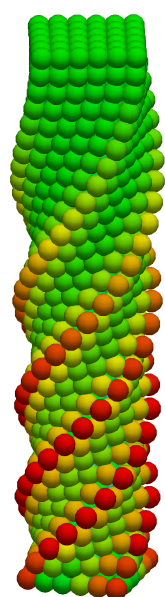

(c) $6 \times 6 \times 31$

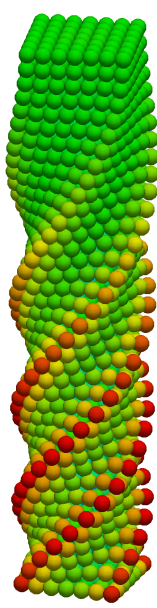

(d) $7 \times 7 \times 37$

$-2 e+06$ $-1.4 e+6$ $-7 e+5$ $7 e+5$ $1,4 e+6$ $2 e+06$

\section{Pressure $(\mathrm{Pa})$}

Figure 25: Twisting column: A sequence of particle refinement analysis using the mixed-based $\{\boldsymbol{p}, \boldsymbol{F}\}$ JST-SPH: (a) $4 \times 19 \times 4$; (b) $5 \times 25 \times 5$; (c) $6 \times 31 \times 6$; and (d) $7 \times 37 \times 7$ particles. Results obtained with an angular velocity field $\boldsymbol{\omega}_{0}=[0, \Omega \sin (\pi Y / 2 L), 0]$ where $\Omega=105 \mathrm{rad} / \mathrm{s}$ and $L=6 \mathrm{~m}$. A neo-Hookean material is used with density $\rho_{0}=1100 \mathrm{~kg} / \mathrm{m}^{3}$, Young's modulus $E=17$ $\mathrm{MPa}$, Poisson's ratio $\nu=0.45$ and $\alpha_{C F L}=0.3$. JST parameters used: $\varepsilon_{\boldsymbol{p}}^{(2)}=0$ and $\varepsilon_{\boldsymbol{p}}^{(4)}=\frac{1}{8}$. 


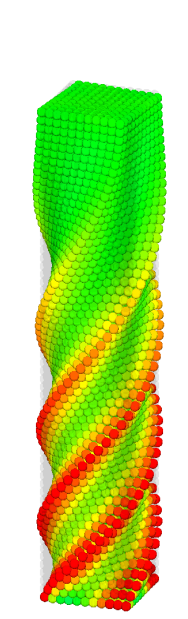

(a)

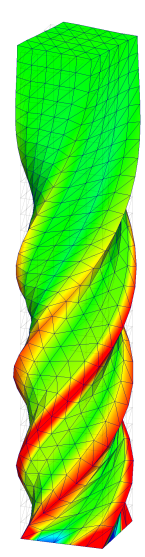

(b)

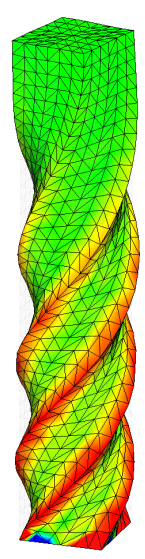

(c)

$$
t=0.1 \mathrm{~s}
$$

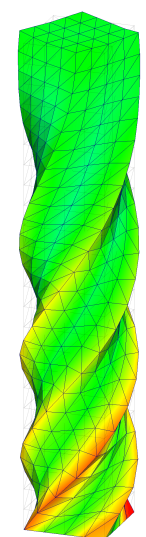

(d)

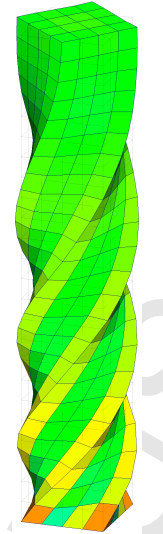

(e)

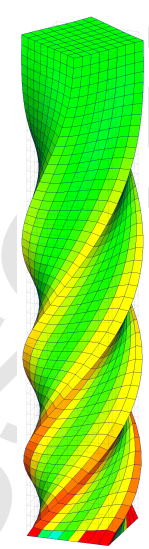

(f)

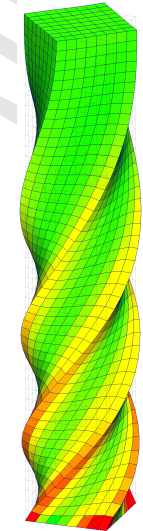

(g)

$$
t=0.25 \mathrm{~s}
$$

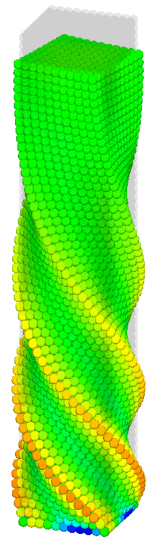

(a)

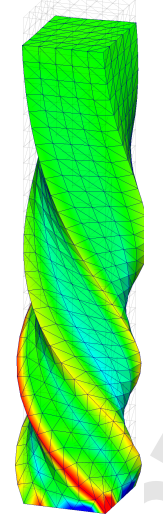

(b)

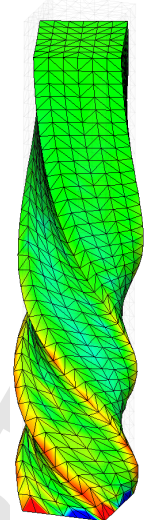

(c)

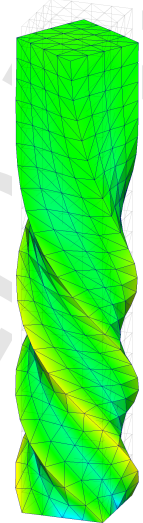

(d)

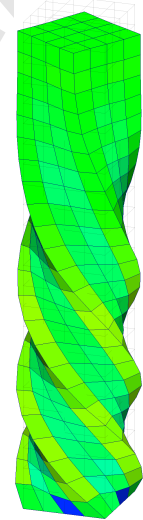

(e)

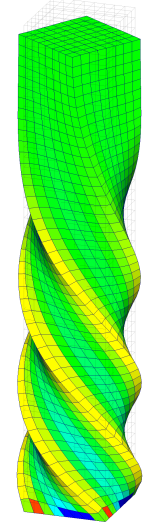

(f)

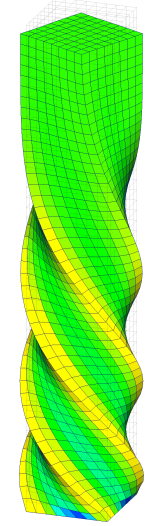

(g)

Figure 26: Twisting column: Comparison of deformed shapes plotted with pressures at time $t=0.1 \mathrm{~s}$ and $t=0.25 \mathrm{~s}$ using: (a) Mixed-based $\{\boldsymbol{p}, \boldsymbol{F}\}$ JSTSPH $\left(\varepsilon_{\boldsymbol{p}}^{(2)}=0\right.$ and $\varepsilon_{\boldsymbol{p}}^{(4)}=\frac{1}{8}$ ); (b) Upwind-VCFVM [44]; (c) JST-VCFVM [43]; (d) Hu-Washizu type variational principle [49]; (e) B-bar hexahedral method [8]; (f) Constrained-TOUCH \$36]; and (g) Hyperelastic-GLACE [47]. Results obtained with an angular velocity field $\boldsymbol{\omega}_{0}=[0, \Omega \sin (\pi Z / 2 L), 0]$ where $\Omega=105 \mathrm{rad} / \mathrm{s}$ and $L=6 \mathrm{~m}$. A neo-Hookean material is used with density $\rho_{0}=1100 \mathrm{~kg} / \mathrm{m}^{3}$, Young's modulus $E=17 \mathrm{MPa}$ and Poisson's ratio $\nu=0.45$. 

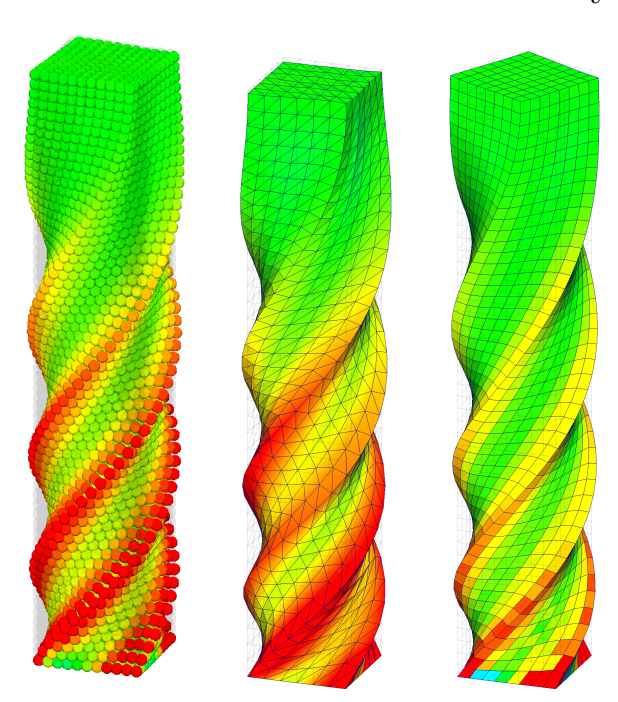

$t=0.1 \mathrm{~s}$

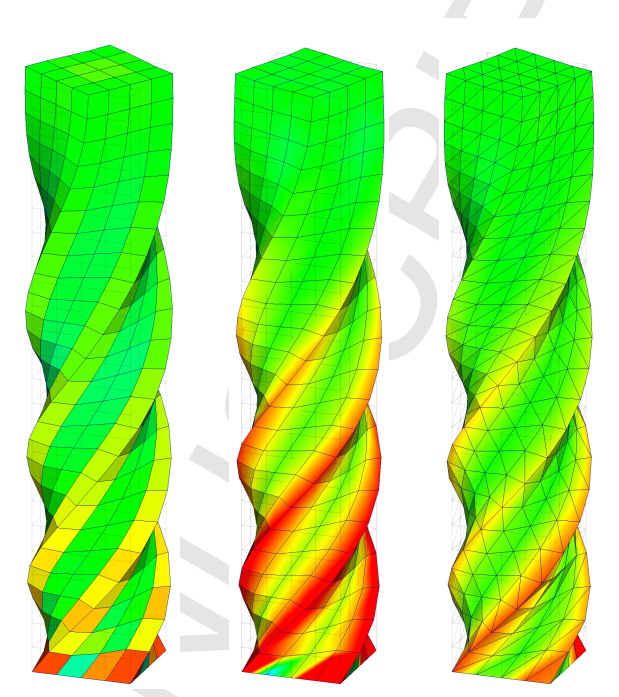

$$
t=0.25 \mathrm{~s}
$$

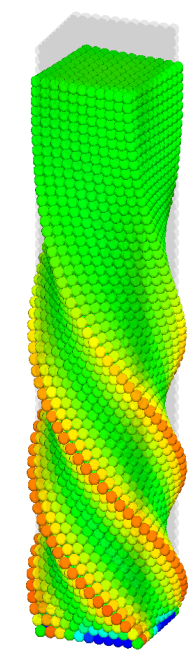

(a)

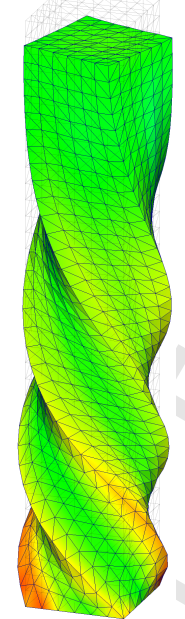

(b)

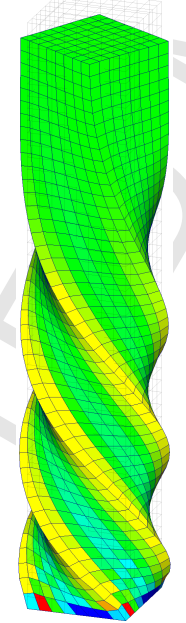

(c)

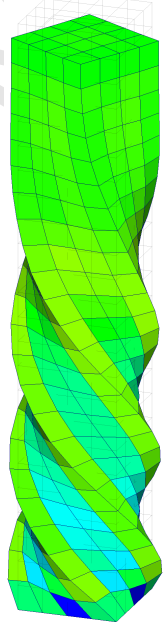

(d)

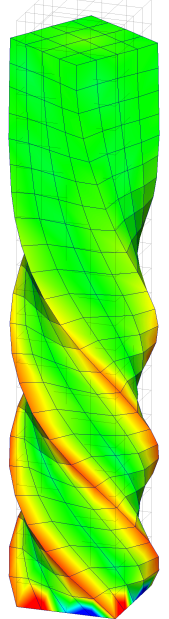

(e)

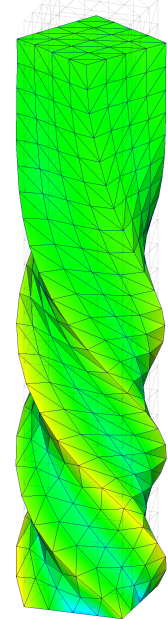

(f)

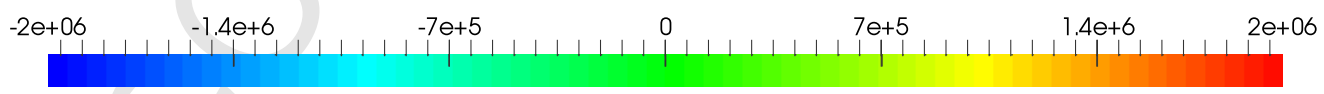

\section{Pressure $(\mathrm{Pa})$}

Figure 27: Twisting column: Comparison of deformed shapes plotted with pressures at time $t=0.1 \mathrm{~s}$ and $t=0.25 \mathrm{~s}$ using: (a) Mixed-based $\{\boldsymbol{p}, \boldsymbol{F}\}$ JSTSPH $\left(\varepsilon_{\boldsymbol{p}}^{(2)}=0\right.$ and $\left.\varepsilon_{\boldsymbol{p}}^{(4)}=\frac{1}{8}\right)$; (b) PG-FE2M [42]; (c) Constrained-TOUCH [36]; (d) B-bar hexahedral method [8]; (e) Taylor-Hood (Q2-Q1) hexahedral FEM [69]; and (f) Hu-Washizu type variational principle [49]. Results obtained with an angular velocity field $\boldsymbol{\omega}_{0}=[0, \Omega \sin (\pi Y / 2 L), 0]$ where $\Omega=105 \mathrm{rad} / \mathrm{s}$ and $L=6 \mathrm{~m}$. A neo-Hookean material is used with density $\rho_{0}=1100 \mathrm{~kg} / \mathrm{m}^{3}$, Young's modulus $E=17 \mathrm{MPa}$ and Poisson's ratio $\nu=0.495$. 


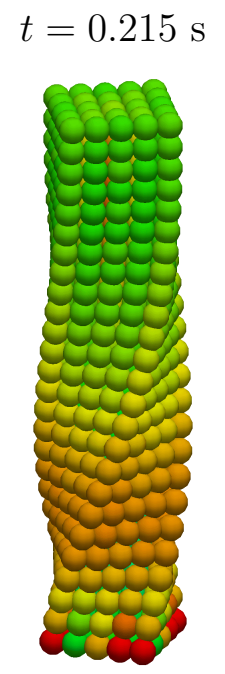

$$
t=0.0407 \mathrm{~s}
$$

$$
t=0.058 \mathrm{~s}
$$

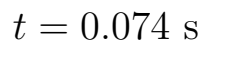

$t=0.09 \mathrm{~s}$

(a) Mixed-based $\{\boldsymbol{p}, \boldsymbol{F}\} \operatorname{JST}-\mathrm{SPH}\left(\varepsilon_{\boldsymbol{p}}^{(2)}=0\right.$ and $\left.\varepsilon_{\boldsymbol{p}}^{(4)}=\frac{1}{8}\right)$
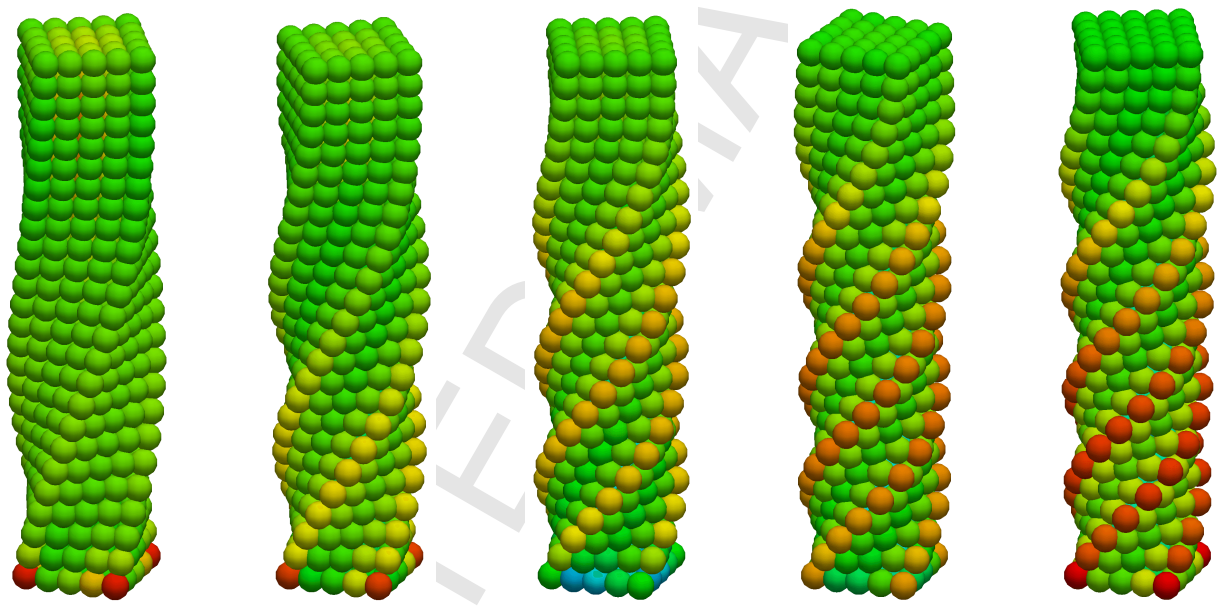

(b) Mixed-based $\{\boldsymbol{p}, \boldsymbol{F}, J\}$ JST-SPH $\left(\varepsilon_{\boldsymbol{p}}^{(2)}=\varepsilon_{J}^{(2)}=0, \varepsilon_{\boldsymbol{p}}^{(4)}=\frac{1}{8}\right.$ and $\left.\varepsilon_{J}^{(4)}=\frac{1}{32}\right)$ $-2 e+06$ $-1.4 e+6$ $-7 e+5$
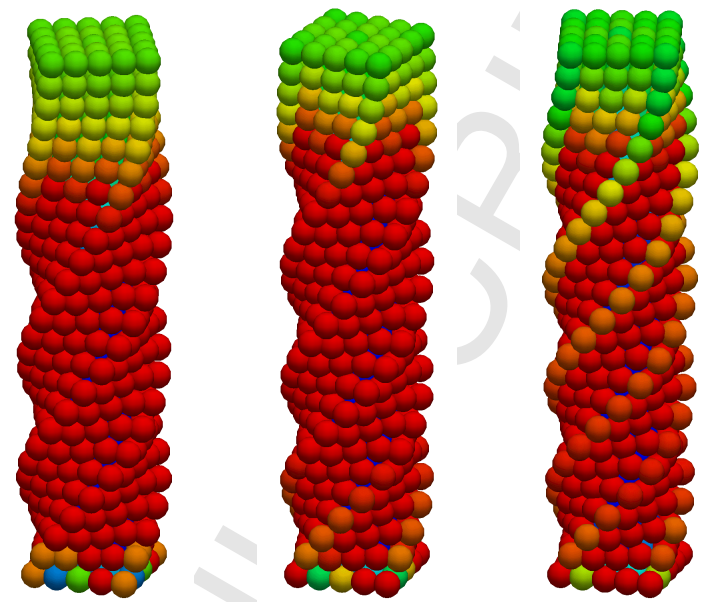

$2 \mathrm{e}+06$

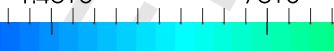

\section{Pressure $(\mathrm{Pa})$}

Figure 28: Twisting column: Comparison of deformed shapes plotted with pressures using (a) Mixed-based $\{\boldsymbol{p}, \boldsymbol{F}\}$ JST-SPH; and (b) Mixed-based $\{\boldsymbol{p}, \boldsymbol{F}, J\}$ JST-SPH. Results obtained with an angular velocity field $\boldsymbol{\omega}_{0}=$ $[0, \Omega \sin (\pi Y / 2 L), 0]$ where $\Omega=105 \mathrm{rad} / \mathrm{s}$ and $L=6 \mathrm{~m}$. A neo-Hookean material is used with density $\rho_{0}=1100 \mathrm{~kg} / \mathrm{m}^{3}$, Young's modulus $E=17$ $\mathrm{MPa}$, Poisson's ratio $\nu=0.499$ and $\alpha_{53} F L=0.3$. Discretisation of $5 \times 25 \times 5$ particles. 
From the spatial discretisation point of view, the very efficient globally conservative Jameson-Schmidt-Turkel (JST) stabilisation algorithm is introduced by taking advantage of the conservative structure of the mixed-based set of equations. Crucially, both velocities and stresses (i.e. deviatoric contribution and volumetric contribution) display the same rate of convergence. In this paper, it has been shown that the $\{\boldsymbol{p}, \boldsymbol{F}\}$ JST-SPH framework performs extremely well in nearly incompressible bending dominated scenarios for a value of Poisson's ratio $\nu \leq 0.495$. For extremely large values of the Poisson's ratio beyond $\nu>0.495$, the enhanced $\{\boldsymbol{p}, \boldsymbol{F}, J\}$ and $\{\boldsymbol{p}, \boldsymbol{F}, \boldsymbol{H}, J\}$ JST-SPH frameworks have been proved to be very efficient in alleviating non-physical hydrostatic pressure fluctuations. More interestingly, practically identical results can be obtained with and without the exact enforcement of essential boundary conditions.

From the temporal discretisation point of view, a monolithic one-step two-stage Total Variation Diminishing Runge Kutta time integrator combined with an angular momentum preserving algorithm is presented. Finally, a comprehensive list of challenging numerical examples has been presented in order to benchmark the results obtained against alternative numerical strategies, including implicit and explicit time integrators as well as Finite Element and Finite Volume based discretisations. The proposed JST-SPH schemes show excellent behaviour in the near incompressibility regime accompanied by severe distortions.

In subsequent publications, the authors will build upon the current work in order to explore three new aspects: first, the consideration of an alternative residual-based dissipative mechanism widely known as Petrov-Galerkin stabilisation [41]; second, the consideration of non-isothermal materials [44] and third, an adaptation of the current computational framework to highstrain ductile fracture application [72] incorporating an alternative Updated Lagrangian formalism in the style of [17].

\section{Acknowledgements}

The authors gratefully acknowledge the financial support provided by the Sêr Cymru National Research Network for Advanced Engineering and Materials, United Kingdom. 


\section{Appendix A. Laplacian correction}

As discussed in Section 3.4, the Jameson-Schmidt-Turkel (JST) stabilisation algorithm employed in this paper requires the evaluation of the Laplacian of the solution function. Taking inspiration from the work of Bonet and Kulasegaram [30], a local correction can be directly introduced into the kernel Laplacian (already presented in equation (24), but repeated here for convenience)

$$
\mathcal{L}\left[\boldsymbol{f}\left(\boldsymbol{X}_{a}\right)\right] \approx \sum_{b \in \Lambda_{a}^{b}} V_{b}\left(\boldsymbol{f}_{b}-\boldsymbol{f}_{a}\right) \tilde{\Delta}_{0} \mathrm{~W}_{b}\left(\boldsymbol{X}_{a}\right) .
$$

Here, the Laplacian correction is defined in terms of two new correction terms $\left\{\boldsymbol{\alpha}_{a}, \beta_{a}\right\}$, that is

$$
\tilde{\Delta}_{0} \mathrm{~W}_{b}\left(\boldsymbol{X}_{a}\right)=\Delta_{0} \mathrm{~W}_{b}\left(\boldsymbol{X}_{a}\right)+\boldsymbol{\alpha}_{a} \cdot \boldsymbol{r}_{a b}+\beta_{a}\left\|\boldsymbol{r}_{a b}\right\|^{2}
$$

with $\boldsymbol{r}_{a b}=\boldsymbol{X}_{b}-\boldsymbol{X}_{a}$. The correction parameters can now be evaluated by enforcing the following two conditions:

1. The Laplacian of a linear function must vanish, that is

$$
\sum_{b \in \Lambda_{a}^{b}} V_{b} \boldsymbol{r}_{a b} \tilde{\Delta}_{0} \mathrm{~W}_{b}\left(\boldsymbol{X}_{a}\right)=\mathbf{0}
$$

2. The Laplacian of a quadratic function gives:

$$
\frac{1}{6} \sum_{b \in \Lambda_{a}^{b}} V_{b}\left\|\boldsymbol{r}_{a b}\right\|^{2} \tilde{\Delta}_{0} \mathrm{~W}_{b}\left(\boldsymbol{X}_{a}\right)=1
$$

By substituting expression (A.2) into (A.3) and (A.4), the correction parameters $\left\{\boldsymbol{\alpha}_{a}, \beta_{a}\right\}$ can then be obtained upon solution of the following system of equations:

$$
\left[\begin{array}{cc}
\sum_{b \in \Lambda_{a}^{b}} V_{b} \boldsymbol{r}_{a b} \otimes \boldsymbol{r}_{a b} & \sum_{b \in \Lambda_{a}^{b}} V_{b}\left\|\boldsymbol{r}_{a b}\right\|^{2} \boldsymbol{r}_{a b} \\
\left(\sum_{b \in \Lambda_{a}^{b}} V_{b}\left\|\boldsymbol{r}_{a b}\right\|^{2} \boldsymbol{r}_{a b}\right)^{T} & \sum_{b \in \Lambda_{a}^{b}} V_{b}\left\|\boldsymbol{r}_{a b}\right\|^{4}
\end{array}\right]\left[\begin{array}{c}
\boldsymbol{\alpha}_{a} \\
\beta_{a}
\end{array}\right]=\left[\begin{array}{c}
-\sum_{\in \Lambda_{a}^{b}} V_{b} \boldsymbol{r}_{a b} \Delta_{0} \mathrm{~W}_{b}\left(\boldsymbol{X}_{a}\right) \\
6-\sum_{\in \Lambda_{a}^{b}} V_{b}\left\|\boldsymbol{r}_{a b}\right\|^{2} \Delta_{0} \mathrm{~W}_{b}\left(\boldsymbol{X}_{a}\right)
\end{array}\right] .
$$




\section{Appendix B. Essential boundary conditions}

As reported in References [17-19], exact enforcement of essential (or Dirichlet) boundary conditions is not strictly essential when employing a SPH discretisation. However, and for completeness, special treatment of essential boundary conditions through the use of a Lagrange multiplier method $[30,55]$ is presented in this paper.

Following References $[30,55]$, the time rate of change of the nodal linear momentum $\dot{\boldsymbol{p}}_{a}$ is modified (in a least-square sense) in order to precisely enforce essential boundary conditions, whilst still ensuring the global conservation of the linear and angular momenta. Inevitably, corrections are only applied to a small number of particles $a$ that lie inside a sphere of a given radius of possible boundary particles $B$. This leads to the following conditions to be fully fulfilled:

$$
\dot{\boldsymbol{p}}_{B}^{\text {Pres }}=\dot{\boldsymbol{p}}\left(\boldsymbol{X}_{B}\right):=\sum_{a \in \Lambda_{B}^{a}} V_{a} \hat{\dot{\boldsymbol{p}}}_{a} \tilde{\mathrm{W}}_{a}\left(\boldsymbol{X}_{B}\right)
$$

and

$$
\sum_{a} V_{a} \dot{\boldsymbol{p}}_{a}=\sum_{a} V_{a} \hat{\dot{\boldsymbol{p}}}_{a} ; \quad \sum_{a} V_{a} \mathscr{X}_{a} \times \dot{\boldsymbol{p}}_{a}=\sum_{a} V_{a} \mathscr{X}_{a} \times \hat{\dot{\boldsymbol{p}}}_{a}
$$

A least-square minimisation procedure is used to obtain modified $\hat{\dot{\boldsymbol{p}}}_{a}$ that satisfies the above conditions (B.1) and (B.2). This can be achieved by computing the minimum of the following functional $\Pi_{E}$ (ignoring time arguments for brevity):

$$
\begin{aligned}
\Pi_{E}\left(\hat{\dot{\boldsymbol{p}}}_{a}, \boldsymbol{\beta}_{B}, \boldsymbol{\beta}_{\mathrm{ang}}, \boldsymbol{\beta}_{\mathrm{lin}}\right) & =\frac{1}{2} \sum_{a} V_{a}\left(\hat{\dot{\boldsymbol{p}}}_{a}-\dot{\boldsymbol{p}}_{a}\right) \cdot\left(\hat{\dot{\boldsymbol{p}}}_{a}-\dot{\boldsymbol{p}}_{a}\right) \\
& +\sum_{B} V_{B} \boldsymbol{\beta}_{B} \cdot\left(\dot{\boldsymbol{p}}_{B}^{\text {Pres }}-\sum_{a \in \Lambda_{B}^{a}} V_{a} \hat{\boldsymbol{p}}_{a} \tilde{\mathrm{W}}_{a}\left(\boldsymbol{X}_{B}\right)\right) \\
& +\boldsymbol{\beta}_{\text {lin }} \cdot\left(\sum_{a} V_{a} \dot{\boldsymbol{p}}_{a}-\sum_{a} V_{a} \hat{\dot{\boldsymbol{p}}}_{a}\right) \\
& +\boldsymbol{\beta}_{\mathrm{ang}} \cdot\left(\sum_{a} V_{a} \mathscr{X}_{a} \times \dot{\boldsymbol{p}}_{a}-\sum_{a} V_{a} \mathscr{X}_{a} \times \hat{\dot{\boldsymbol{p}}}_{a}\right)
\end{aligned}
$$


where $\dot{\boldsymbol{p}}_{B}^{\text {Pres }}$ denotes possible prescribed boundary conditions and $\left\{\boldsymbol{\beta}_{B}, \boldsymbol{\beta}_{\text {lin }}, \boldsymbol{\beta}_{\text {ang }}\right\}$ are the corresponding set of Lagrange multipliers. Differentiating $\Pi_{E}$ with respect to $\hat{\dot{\boldsymbol{p}}}_{a}$ gives:

$$
\hat{\dot{\boldsymbol{p}}}_{a}=\dot{\boldsymbol{p}}_{a}+\sum_{B \in \Lambda_{a}^{B}} V_{B} \tilde{\mathrm{W}}_{a}\left(\boldsymbol{X}_{B}\right) \boldsymbol{\beta}_{B}+\boldsymbol{\beta}_{\mathrm{ang}} \times \mathscr{X}_{a}+\boldsymbol{\beta}_{\mathrm{lin}} .
$$

Substitution of the above expression (B.4) into (B.1) and (B.2) leads to the following system of equations for $\left\{\boldsymbol{\beta}_{B}, \boldsymbol{\beta}_{\text {lin }}, \boldsymbol{\beta}_{\text {ang }}\right\}$, that is

$$
\boldsymbol{L} \boldsymbol{\beta}=\boldsymbol{R} ; \quad \boldsymbol{\beta}=\left[\begin{array}{c}
\boldsymbol{\beta}_{B} \\
\boldsymbol{\beta}_{\text {lin }} \\
\boldsymbol{\beta}_{\text {ang }}
\end{array}\right] ; \quad \boldsymbol{R}=\left[\begin{array}{c}
\dot{\boldsymbol{p}}_{D}^{\text {Pres }}-\dot{\boldsymbol{p}}\left(\boldsymbol{X}_{D}\right) \\
\mathbf{0} \\
\mathbf{0}
\end{array}\right]
$$

with

$$
\boldsymbol{L}=\left[\begin{array}{ccc}
\sum_{a \in \Lambda_{D}^{a}} V_{a} \tilde{\mathrm{W}}_{a}\left(\boldsymbol{X}_{D}\right)\left[\sum_{B \in \Lambda_{a}^{B}} V_{B} \tilde{\mathrm{W}}_{a}\left(\boldsymbol{X}_{B}\right)\right] & 1 & -\sum_{a} V_{a} \tilde{\mathrm{W}}_{a}\left(\boldsymbol{X}_{D}\right) \hat{\mathscr{X}}_{a} \\
\sum_{B} V_{B} & \sum_{a} V_{a} & -\sum_{a} V_{a} \hat{\mathscr{X}}_{a} \\
\sum_{B} V_{B}\left[\sum_{a \in \Lambda_{B}^{a}} V_{a} \tilde{\mathrm{W}}_{a}\left(\boldsymbol{X}_{B}\right) \hat{\mathscr{X}}_{a}\right] & \sum_{a} V_{a} \hat{\mathscr{X}}_{a} & \sum_{a} V_{a}\left[\left(\mathscr{X}_{a} \cdot \mathscr{X}_{a}\right) \boldsymbol{I}-\mathscr{X}_{a} \otimes \mathscr{X}_{a}\right]
\end{array}\right] .
$$

\section{Appendix C. Numerical errors: Dispersion and diffusion}

Considering a one dimensional linear constant advection equation

$$
\frac{\partial u}{\partial t}+c \frac{\partial u}{\partial x}=0
$$

where $c$ describes the constant positive wave speed and $u$ represents the unknown variable. Following the SPH spatial discretisation described in Section 3 , the discrete counterpart in particle $a$ of above equation is

$$
\frac{d u_{a}}{d t}=\underbrace{-\frac{c}{2 h_{\min }}\left(u_{a+1}^{n}-u_{a-1}^{n}\right)-\frac{c \varepsilon^{(4)}}{h_{\min }}\left(u_{a+2}^{n}-4 u_{a+1}^{n}+6 u_{a}^{n}-4 u_{a-1}^{n}+u_{a-2}^{n}\right)}_{\mathcal{R}\left(u_{\{a+2, a+1, a, a-1, a-2\}}^{n}\right)} .
$$

Here, $h_{\min }$ represents the spacing between particles and $\varepsilon^{(4)}$ is the biharmonic JST parameter. Notice that 2 neighbouring particles on each side are employed in the evaluation of the gradient and Laplacian operators. 
Expression above can now be integrated in time using the Total Variation Diminishing Runge Kutta (TVD-RK) time integrator (43), that is:

$$
u_{a}^{n+1}=u_{a}^{n}+\frac{\Delta t}{2}\left[\mathcal{R}\left(u_{\{a+2, a+1, a, a-1, a-2\}}^{n}\right)+\mathcal{R}\left(u_{\{a+2, a+1, a, a-1, a-2\}}^{\star}\right)\right] .
$$

Using a Taylor series expansion procedure, the above terms, after some algebraic manipulation, are expanded about $\left(x_{a}, t^{n}\right)$ to give:

$$
\frac{\partial u}{\partial t}+c \frac{\partial u}{\partial x}=\underbrace{\left[\left(\alpha_{C F L}^{2}-1\right) \frac{c h_{\min }^{2}}{6}\right]}_{\text {Numerical dispersion }} u_{x x x}+\underbrace{\left[\left(\frac{\alpha_{C F L}}{6}-\frac{\alpha_{C F L}^{3}}{24}-\varepsilon^{(4)}\right) c h_{\min }^{3}\right]}_{\text {Numerical diffusion }} u_{x x x x}+\mathcal{O}\left(h^{4}\right) .
$$

It is worthwhile pointing out that the resulting dispersion and diffusion errors in (C.4) match those obtained with the JST-based Finite Volume Method reported in Reference [43].

[1] J. Bonet, A. J. Gil, C. H. Lee, M. Aguirre, R. Ortigosa, A first order hyperbolic framework for large strain computational solid dynamics. Part I: Total Lagrangian isothermal elasticity, Computer Methods in Applied Mechanics and Engineering 283 (2015) 689-732.

[2] A. J. Gil, C. H. Lee, J. Bonet, R. Ortigosa, A first order hyperbolic framework for large strain computational solid dynamics. Part II: Total Lagrangian compressible, nearly incompressible and truly incompressible elasticity, Computer Methods in Applied Mechanics and Engineering 300 (2016) 146-181.

[3] A. Jameson, W. Schmidt, E. Turkel, Numerical solution of the Euler equations by finite volume methods using Runge Kutta time stepping schemes, AIAA 14th Fluid and Plasma Dynamic Conference 81 (1981).

[4] J. O. Hallquist, LS-DYNA theoretical manual, Livermore Software Technology Corporation (1998).

[5] J. O. Hallquist, LS-DYNA keyword user's manual, Livermore Software Technology Corporation (2003).

[6] Hibbitt, Karlsson, Sorensen, ABAQUS/Explicit: User's manual (volume 1, version 6.1) (2000). 
[7] D. P. Flanagan, T. Belytschko, A uniform strain hexahedron and quadrilateral with orthogonal hourglass control, International Journal for $\mathrm{Nu}$ merical Methods in Engineering 17 (1981) 679-706.

[8] T. J. R. Hughes, Generalization of selective integration procedures to anisotropic and nonlinear media, International Journal for Numerical Methods in Engineering 15 (1980) 1413-1418.

[9] J. Bonet, H. Marriott, O. Hassan, An averaged nodal deformation gradient linear tetrahedral element for large strain explicit dynamic applications, Communications in Numerical Methods in Engineering 17 (2001) $551-561$.

[10] Y. Onishi, K. Amaya, A locking-free selective smoothed finite element method using tetrahedral and triangular elements with adaptive mesh rezoning for large deformation problems, International Journal for Numerical Methods in Engineering 99 (2014) 354-371.

[11] N. P. Weatherill, O. Hassan, Efficient three-dimensional Delaunay triangulation with automatic point creation and imposed boundary constraints, International Journal for Numerical Methods in Engineering 37 (1994) 2005-2039.

[12] S. K. Lahiri, J. Bonet, J. Peraire, A variationally consistent mesh adaptation method for triangular elements in explicit Lagrangian dynamics, International Journal for Numerical Methods in Engineering 82 (2010) 1073-1113.

[13] L. B. Lucy, A numerical approach to the testing of the fission hypothesis, Astronomical Journal 82 (1977) 1013-1024.

[14] R. A. Gingold, J. J. Monaghan, Smoothed Particle HydrodynamicsTheory and application to non-spherical stars, Monthly Notices of the Royal Astronomical Society 181 (1977) 375-389.

[15] P. W. Randles, L. D. Libersky, Smoothed Particle Hydrodynamics: Some recent improvements and applications, Computer Methods in Applied Mechanics and Engineering 139 (1996) 375-408. 
[16] G. R. Johnson, S. R. Beissel, Normalized smoothing functions for SPH impact computations, International Journal for Numerical Methods in Engineering 39 (1996) 2725-2741.

[17] Y. Vidal, J. Bonet, A. Huerta, Stabilized updated Lagrangian corrected SPH for explicit dynamic problems, International Journal for Numerical Methods in Engineering 69 (2006) 2687-2710.

[18] J. Bonet, S. Kulasegaram, Remarks on tension instability of Eulerian and Lagrangian Corrected Smooth Particle Hydrodynamics (CSPH) methods, International Journal for Numerical Methods in Engineering 52 (2001) 1203-1220.

[19] G. C. Ganzenmüller, An hourglass control algorithm for Lagrangian Smooth Particle Hydrodynamics, Computer Methods in Applied Mechanics and Engineering 286 (2015) 87-106.

[20] J. W. Swegle, D. L. Hicks, S. W. Attaway, Smoothed Particle Hydrodynamics stability analysis, Journal of Computational Physics 116 (1995) $123-134$.

[21] T. Belytschko, Y. Guo, W. K. Liu, S. P. Xiao, A unified stability analysis of meshless particle methods, International Journal for Numerical Methods in Engineering 48 (2000) 1359-1400.

[22] J. J. Monaghan, SPH without a tensile instability, Journal of Computational Physics 159 (2000) 290-311.

[23] M. A. Puso, J. S. Chen, E. Zywicz, W. Elmer, Meshfree and finite element nodal integration methods, International Journal for Numerical Methods in Engineering 74 (2008) 416-446.

[24] J. Bonet, A. J. Burton, A simple average nodal pressure tetrahedral element for incompressible and nearly incompressible dynamic explicit applications, Communications in Numerical Methods in Engineering 14 (1998) 437-449.

[25] M. W. Gee, C. R. Dohrmann, S. W. Key, W. A. Wall, A uniform nodal strain tetrahedron with isochoric stabilization, International Journal for Numerical Methods in Engineering 78 (2009) 429-443. 
[26] C. R. Dohrmann, M. W. Heinstein, J. Jung, S. W. Key, W. R. Witkowski, Node-based uniform strain elements for three-node triangular and four-node tetrahedral meshes, International Journal for $\mathrm{Nu}-$ merical Methods in Engineering 47 (2000) 1549-1568.

[27] M. A. Puso, J. Solberg, A stabilized nodally integrated tetrahedral, International Journal for Numerical Methods in Engineering 67 (2006) $841-867$.

[28] M. B. Liu, G. R. Liu, K. Y. Lam, Constructing smoothing functions in smoothed particle hydrodynamics with applications, Jounal of Computational and Applied Mathematics 155 (2003) 263-284.

[29] J. K. Chen, J. E. Beraun, T. C. Carney, A corrective smoothed particle method for boundary value problems in heat conduction, International Journal for Numerical Methods in Engineering 46 (1999) 231-252.

[30] J. Bonet, S. Kulasegaram, Correction and stabilization of smooth particle hydrodynamics methods with applications in metal forming simulations, International Journal for Numerical Methods in Engineering 47 (2000) 1189-1214.

[31] T. Blanc, M. Pastor, A stabilized Smoothed Particle Hydrodynamics, Taylor-Galerkin algorithm for soil dynamics problems, International Journal for Numerical and Analytical Methods in Engineering 37 (2013) $1-30$.

[32] M. I. Herreros, M. Mabssout, A two-steps time discretization scheme using the SPH method for shock wave propogation, Computer Methods in Applied Mechanics and Engineering 200 (2011) 1833-1845.

[33] M. Mabssout, M. I. Herreros, Runge-Kutta vs Taylor-SPH: Two time integration schemes for SPH with application to soil dynamics, Applied Mathematical Modelling 37 (2013) 3541-3563.

[34] G. C. Ganzenmüller, S. Hiermaier, M. May, On the similarity of meshless discretizations of Peridynamics and Smooth-Particle Hydrodynamics, Computers and Structures 150 (2015) 71-78.

[35] E. F. Toro, Riemann solvers and numerical methods for fluid dynamics: A practical introduction, Springer-Verlag, second edition, 2006. 
[36] J. Haider, C. H. Lee, A. J. Gil, J. Bonet, A first order hyperbolic framework for large strain computational solid dynamics: An upwind cell centred Total Lagrangian scheme, International Journal for Numerical Methods in Engineering (2016). DOI: 10.1002/nme.5293.

[37] C. H. Lee, A. J. Gil, J. Bonet, Development of a cell centred upwind finite volume algorithm for a new conservation law formulation in structural dynamics, Computers and Structures 118 (2013) 13-38.

[38] A. J. Gil, Z. Zhang, O. Hassan, K. Morgan, Parallel multigrid detached eddy simulation algorithm for three-dimensional unsteady incompressible flows on unstructured grids, Journal of Aerospace Engineering 19 (2006) 271-280.

[39] J. A. Trangenstein, A second-order Godunov algorithm for twodimensional solid mechanics, Computational Mechanics 13 (1994) 343359 .

[40] J. A. Trangenstein, P. Colella, A higher-order Godunov method for modelling finite deformation in elastic-plastic solids, Communications on Pure and Applied Mathematics 44 (1991) 41-100.

[41] C. H. Lee, A. J. Gil, J. Bonet, Development of a stabilised PetrovGalerkin formulation for conservation laws in Lagrangian fast solid dynamics, Computer Methods in Applied Mechanics and Engineering 268 (2014) 40-64.

[42] A. J. Gil, C. H. Lee, J. Bonet, M. Aguirre, A stabilised Petrov-Galerkin formulation for linear tetrahedral elements in compressible, nearly incompressible and truly incompressible fast dynamics, Computer Methods in Applied Mechanics and Engineering 276 (2014) 659-690.

[43] M. Aguirre, A. J. Gil, J. Bonet, A. A. Carreño, A vertex centred finite volume Jameson-Schmidt-Turkel (JST) algorithm for a mixed conservation formulation in solid dynamics, Journal of Computational Physics 259 (2014) 672-699.

[44] M. Aguirre, A. J. Gil, J. Bonet, C. H. Lee, An upwind vertex centred finite volume solver for Lagrangian solid dynamics, Journal of Computational Physics 300 (2015) 387-422. 
[45] I. A. Karim, C. H. Lee, A. J. Gil, J. Bonet, A two-step Taylor Galerkin formulation for fast dynamics, Engineering Computations 31 (2014) $366-387$.

[46] N. C. Nguyen, J. Peraire, Hybridizable Discontinuous Galerkin methods for partial differential equations in continuum mechanics, Journal of Computational Physics 231 (2012) 5955-5988.

[47] G. Kluth, B. Després, Discretization of hyperelasticity on unstructured mesh with a cell-centered Lagrangian scheme, Journal of Computational Physics 229 (2010) 9092-9118.

[48] J. Bonet, A. J. Gil, R. Ortigosa, On a tensor cross product based formulation of large strain solid mechanics, International Journal of Solids and Structures 84 (2016) 49-63.

[49] J. Bonet, A. J. Gil, R. Ortigosa, A computational framework for polyconvex large strain elasticity, Computer Methods in Applied Mechanics and Engineering 283 (2015) 1061-1094.

[50] C. M. Dafermos, Quasilinear hyperbolic systems with involutions, Archive for Rational Mechanics and Analysis 94 (1986) 373-389.

[51] J. E. Marsden, T. J. R. Hughes, Mathematical foundations of elasticity, Dover Publications, 1994.

[52] J. Bonet, T.-S. Lok, Variational and momentum preservation aspects of smooth particle hydrodynamic formulations, Computer Methods in Applied Mechanics and Engineering 180 (1999) 97-115.

[53] J. J. Monaghan, Smoothed particle hydrodynamics, Annual Review of Astronomy and Astrophysics 30 (1992) 543-574.

[54] A. Huerta, S. Fernández-Méndez, Enrichment and coupling of the finite element and meshless methods, International Journal for Numerical Methods in Engineering 48 (2000) 1615-1636.

[55] S. Fernández-Méndez, A. Huerta, Imposing essential boundary conditions in mesh-free methods, Computer Methods in Applied Mechanics and Engineering 193 (2004) 1257-1275. 
[56] A. Huerta, S. Fernández-Méndez, Locking in the incompressible limit for the element-free Galerkin method, International Journal for Numerical Methods in Engineering 51 (2001) 1361-1383.

[57] K. J. Bathe, Finite element procedures, Prentice Hall, 1996.

[58] S. Fernández-Méndez, A. Huerta, Continuous blending of SPH with finite elements, Computes and Structures 83 (2005) 1448-1458.

[59] J. P. Gray, J. J. Monaghan, R. P. Swift, SPH elastic dynamics, Computer Methods in Applied Mechanics and Engineering 190 (2001) 66416662 .

[60] R. Vignjevic, J. Campbell, L. Libersky, A treatment of zero-energy modes in the smoothed particle hydrodynamics method, Computer Methods in Applied Mechanics and Engineering 184 (2000) 67-85.

[61] A. J. C. Crespo, J. M. Domínguez, B. D. Rogers, M. Gómez-Gesteira, S. Longshaw, R. Canelas, R. Vacondio, A. Barreiro, O. García-Feal, DualSPHysics: Open-source parallel CFD solver based on Smoothed Particle Hydrodynamics (SPH), Computer Physics Communications 187 (2015) 204-216.

[62] A. Mokos, B. D. Rogers, P. K. Stansby, J. M. Domínguez, Multi-phase SPH modelling of violent hydrodynamics on GPUs, Computer Physics Communications 196 (2015) 304-316.

[63] O. C. Zienkiewicz, J. Rojek, R. L. Taylor, M. Pastor, Triangles and tetrahedra in explicit dynamic codes for solids, International Journal for Numerical Methods in Engineering 43 (1998) 565-583.

[64] M. A. Puso, A highly efficient enhanced assumed strain physically stabilized hexahedral element, International Journal for Numerical Methods in Engineering 49 (2000) 1029-1064.

[65] J. S. Chen, C. T. Wu, S. Yoon, Y. You, A stabilized conforming nodal integration for Galerkin mesh-free methods, International Journal for Numerical Methods in Engineering 50 (2001) 435-466. 
[66] M. Hillman, J. S. Chen, An accelerated, convergent, and stable nodal integration in Galerkin meshfree methods for linear and nonlinear mechanics, International Journal for Numerical Methods in Engineering (2015). DOI: 10.1002/nme.5183.

[67] W. Elmer, J. S. Chen, M. Puso, E. Taciroglu, A stable, meshfree, nodal integration method for nearly incompressible solids, Finite Elements in Analysis and Design 51 (2012) 81-85.

[68] R. Courant, K. Friedrichs, H. Lewy, On the partial difference equations of mathematical physics, Mathematische Annalem 100 (1928) 32-74.

[69] J. Donea, A. Huerta, Finite element methods for flow problems, Wiley and Sons, 2004.

[70] C. T. Dyka, R. P. Ingel, An approach for tension instability in Smoothed Particle Hydrodynamics (SPH), Computers and Structures 57 (1995) $573-580$.

[71] G. Scovazzi, B. Carnes, X. Zeng, S. Rossi, A simple, stable, and accurate linear tetrahedral finite element for transient, nearly and fully incompressible solid dynamics: A dynamic variational multiscale approach, International Journal for Numerical Methods in Engineering (2015). DOI: 10.1002/nme.5138.

[72] K. Moreau, N. Moës, D. Picart, L. Stainer, Explicit dynamics with a non-local damage model using the thick level set approach, International Journal for Numerical Methods in Engineering 102 (2015) 808-838. 